\title{
A COMPARISON OF WRITTEN EXPOSURE WITH AND WITHOUT RESCRIPTING FOR THE TREATMENT OF GENERALIZED ANXIETY DISORDER
}

\author{
by \\ Melina M. Ovanessian \\ Bachelor of Arts, Western University, 2008 \\ A thesis \\ presented to Ryerson University \\ in partial fulfillment of the \\ requirements of the degree of \\ Master of Arts \\ in the program of \\ Psychology
}

Toronto, Ontario, Canada, 2017

(C) Melina M. Ovanessian, 2017 


\section{AUTHOR'S DECLARATION FOR ELECTRONIC SUBMISSION OF A THESIS}

I hereby declare that I am the sole author of this thesis. This is a true copy of the thesis, including any required final revisions, as accepted by my examiners.

I authorize Ryerson University to lend this thesis to other institutions or individuals for the purpose of scholarly research

I further authorize Ryerson University to reproduce this thesis by photocopying or by other means, in total or in part, at the request of other institutions or individuals for the purpose of scholarly research.

I understand that my thesis may be made electronically available to the public 
A Comparison of Written Exposure With and Without Rescripting for the Treatment of Generalized Anxiety Disorder

Melina M. Ovanessian

Master of Arts, 2017

Psychology

Ryerson University

\begin{abstract}
The present study examined whether written exposure enhanced with rescripting is more efficacious in reducing GAD symptoms, worry, and related cognitive difficulties than written exposure to the same worst-case scenario or neutral writing. Adults with GAD $(N=67)$ were randomly assigned to one of three conditions and engaged in 30-minute writing sessions on 3 consecutive days: (1) worst-case scenario exposure; WE (2) written exposure with rescripting; RWE (3) Neutral Control; NC. Participants in both exposure conditions displayed significant reductions in worry and fear of emotions from baseline to 1-month follow-up, but $\mathrm{NC}$ participants did not. Participants in the RWE condition also displayed significant improvements in negative problem orientation, perceived cost of the feared scenario, and coping; participants in the WE or NC did not. Findings suggest that overall, RWE was not superior to WE; both written exposure interventions have strong potential to help individuals who suffer from GAD.
\end{abstract}




\section{Acknowledgements}

There are many people I would like to gratefully acknowledge for their continued support. Foremost, thank you to my advisor, Dr. Naomi Koerner. You are an exemplary researcher and mentor and I have been extremely lucky to learn from you. Your guidance, knowledge and encouragement continuously challenges me to grow as a scientist.

Thank you to Dr. Martin Antony for his guidance through this process. Your feedback and ideas make you an absolutely invaluable member of my thesis committee. Thank you also to Dr. Todd Girard, for graciously agreeing to be part of my examining committee. I would also like to thank Dr. Michel Dugas for his assistance with the design of the rescripting condition.

My sincere gratitude also goes out to my fellow labmates, peers and friends. Thank you for making every day fun, engaging, and memorable. Thank you also to Diana Jin and Shanny Foo for volunteering their time to help with the tremendous amount of data entry for this study. To my family and partner, I truly appreciate your unwavering support and understanding over the last few years.

Finally, thank you to the Canadian Institutes for Health Research for providing me with financial support during my Master's degree. 


\section{Table of Contents}

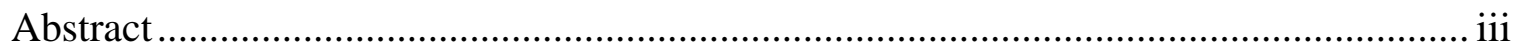

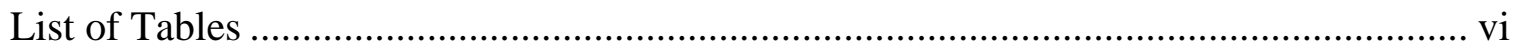

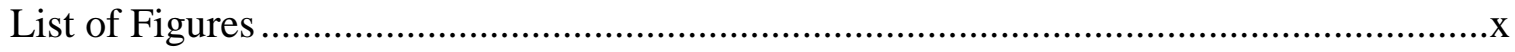

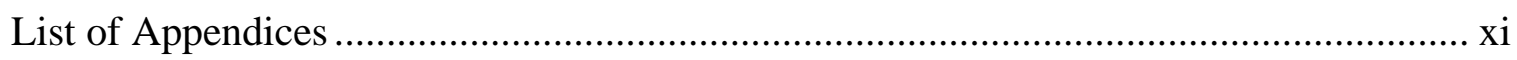

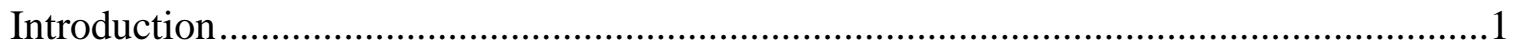

Theories of Generalized Anxiety Disorder .........................................................

Treatment of Generalized Anxiety Disorder...............................................4

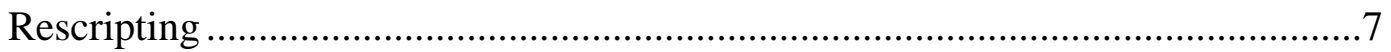

Efficacy of Imagery Rescripting .........................................................

Enhancing Exposure for GAD with Rescripting …...................................10

Present Study: Research Questions and Hypotheses .......................................13

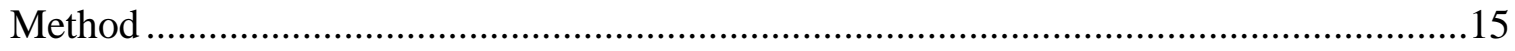

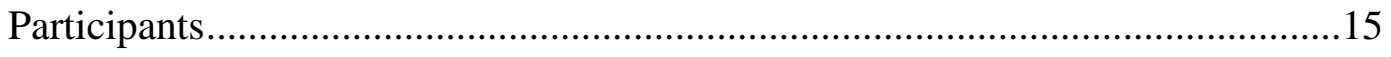

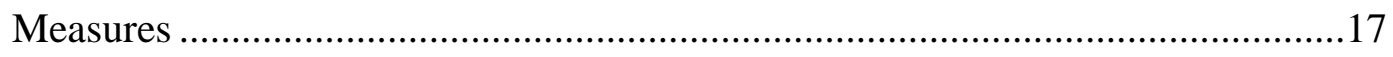

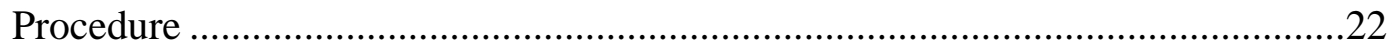

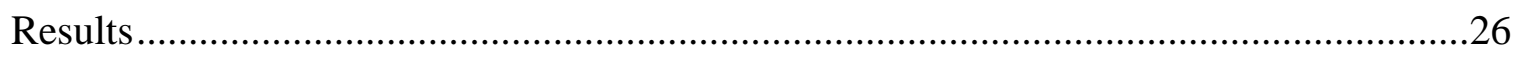

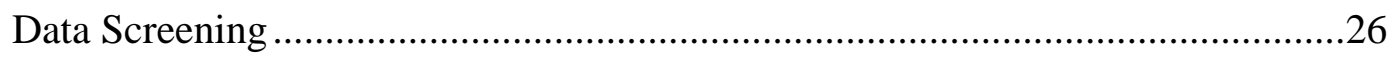

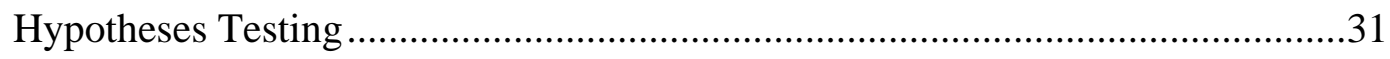

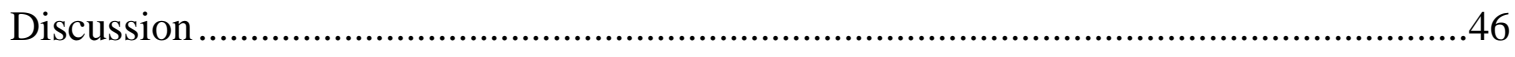

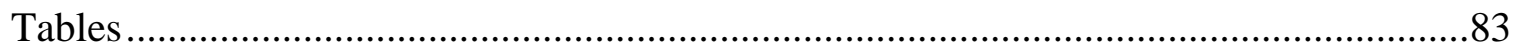

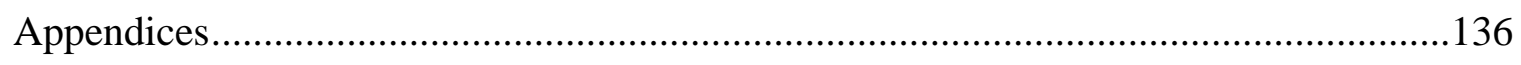

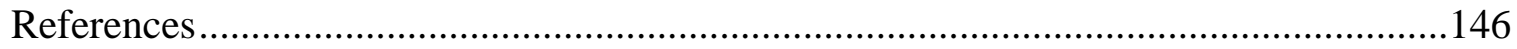




\section{List of Tables}

Table 1: Sample Characteristics Separated by Study Condition ..................................83

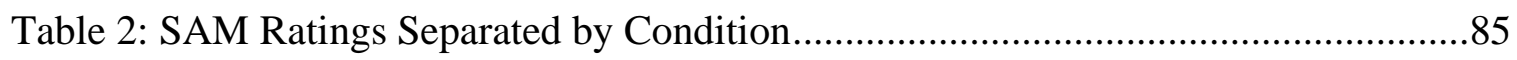

Table 3: Means and Standard Deviations of All Process Variables Separated by Condition

Table 4: Multilevel Models and Piecewise Analyses for PSWQ as Associated with Time and Condition for RWE vs. NC

Table 5: Multilevel Models and Piecewise Analyses for PSWQ as Associated with Time and Condition WE vs. NC

Table 6: Multilevel Models and Piecewise Analyses for PSWQ as Associated with Time and Condition RWE vs. WE. .93

Table 7: Multilevel Models and Piecewise Analyses for GAD-Q-IV Scores as Associated with Time and Condition for RWE vs. NC

Table 8: Multilevel Models and Piecewise Analyses for GAD-Q-IV Scores as Associated with Time and Condition for WE vs. NC . .95

Table 9: Multilevel Models and Piecewise Analyses for GAD-Q-IV Scores as Associated with Time and Condition for RWE vs. WE.... . .96

Table 10: Multilevel Models and Piecewise Analyses for STICSA-T as Associated with Time and Condition for RWE vs. NC

Table 11: Multilevel Models and Piecewise Analyses for STICSA-T as Associated with Time and Condition for WE vs. NC .98

Table 12: Multilevel Models and Piecewise Analyses for STICSA-T as Associated with Time and Condition for RWE vs. WE .99

Table 13: Multilevel Models and Piecewise Analyses for CESD-R as Associated with Time and Condition for RWE vs. NC. 100

Table 14: Multilevel Models and Piecewise Analyses for CESD-R as Associated with Time and Condition for WE vs. NC. 101

Table 15: Multilevel Models and Piecewise Analyses for CESD-R as Associated with Time and Condition for RWE vs. WE 102 
Table 16: Multilevel Models and Piecewise Analyses for IUS as Associated with Time and Condition for RWE vs. NC. 103

Table 17: Multilevel Models and Piecewise Analyses for IUS as Associated with Time and Condition for WE vs. NC. 104

Table 18: Multilevel Models and Piecewise Analyses for IUS as Associated with Time and Condition for RWE vs. WE..... 105

Table 19: Multilevel Models and Piecewise Analyses for CAQ as Associated with Time and Condition for RWE vs. NC. 106

Table 20: Multilevel Models and Piecewise Analyses for CAQ as Associated with Time and Condition for WE vs. NC. 107

Table 21: Multilevel Models and Piecewise Analyses for CAQ as Associated with Time and Condition RWE vs. WE 108

Table 22: Multilevel Models and Piecewise Analyses for NPOQ as Associated with Time and Condition for RWE vs. NC. 109

Table 23: Multilevel Models and Piecewise Analyses for NPOQ as Associated with Time and Condition for WE vs. NC. 110

Table 24: Multilevel Models and Piecewise Analyses for NPOQ as Associated with Time and Condition for RWE vs. WE

Table 25: Multilevel Models and Piecewise Analyses for ACS as Associated with Time and Condition for RWE vs. NC. 112

Table 26: Multilevel Models and Piecewise Analyses for ACS as Associated with Time and Condition for WE vs. NC. 113

Table 27: Multilevel Models and Piecewise Analyses for ACS as Associated with Time and Condition for RWE vs. WE 114

Table 28: Multilevel Models and Piecewise Analyses for ACS Anxiety as Associated with Time and Condition for RWE vs. NC

Table 29: Multilevel Models and Piecewise Analyses for ACS Anxiety as Associated with Time and Condition for WE vs. NC 116

Table 30: Multilevel Models and Piecewise Analyses for ACS Anxiety as Associated with Time and Condition for RWE vs. WE 
Table 31: Multilevel Models and Piecewise Analyses for ACS Anger as Associated with Time and Condition for RWE vs. NC 118

Table 32: Multilevel Models and Piecewise Analyses for ACS Anger as Associated with Time and Condition for WE vs. NC

Table 33: Multilevel Models and Piecewise Analyses for ACS Anger as Associated with Time and Condition for RWE vs. WE 120

Table 34: Multilevel Models and Piecewise Analyses for ACS Depressed Mood as Associated with Time and Condition for RWE vs. NC 121

Table 35: Multilevel Models and Piecewise Analyses for ACS Depressed Mood as Associated with Time and Condition for WE vs. NC

Table 36: Multilevel Models and Piecewise Analyses for ACS Depressed Mood as Associated with Time and Condition for RWE vs. WE

Table 37: Multilevel Models and Piecewise Analyses for ACS Positive Emotion as Associated with Time and Condition for RWE vs. NC. 124

Table 38: Multilevel Models and Piecewise Analyses for ACS Positive Emotion as Associated with Time and Condition for WE vs. NC 125

Table 39: Multilevel Models and Piecewise Analyses for ACS Positive Emotion as Associated with Time and Condition for RWE vs. WE. 126

Table 40: Multilevel Models and Piecewise Analyses and Piecewise Analyses for Perceived Probability Scores Associated with Time and Condition for RWE vs. WE 127

Table 41: Multilevel Models and Piecewise Analyses for Perceived Probability Scores as Associated with Time and Condition for WE vs. NC. 128

Table 42: Multilevel Models and Piecewise Analyses and Piecewise Analyses for Perceived Probability Scores Associated with Time and Condition for RWE vs. WE........129

Table 43: Multilevel Models and Piecewise Analyses for Perceived Cost Scores Associated with Time and Condition for RWE vs. NC. 130

Table 44: Multilevel Models and Piecewise Analyses for Perceived Cost Scores as Associated with Time and Condition for WE vs. NC

Table 45: Multilevel Models and Piecewise Analyses for Perceived Cost Scores as Associated with Time and Condition RWE vs. WE. 
Table 46: Multilevel Models and Piecewise Analyses for Perceived Coping Scores as Associated with Time and Condition for RWE vs. NC........................................................133

Table 47: Multilevel Models and Piecewise Analyses for Perceived Coping Scores as Associated with Time and Condition for WE vs. NC 134

Table 48: Multilevel Models and Piecewise Analyses for Perceived Coping Scores as Associated with Time and Condition for RWE vs. WE 135 


\section{List of Figures}

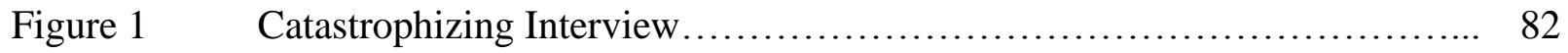




\section{List of Appendices}

Appendix A General Writing Instructions......................................136

Appendix B Worst Case Scenario Writing Instructions.........................137

Appendix C Rescripted Writing Instructions.................................. 138

Appendix D Neutral Writing Instructions...................................139

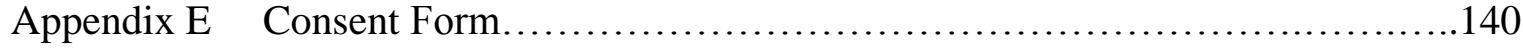

Appendix F Debriefing Form.................................................. 145 
A Comparison of Written Exposure With and Without Rescripting for the Treatment of Generalized Anxiety Disorder

Excessive worry and anxiety are the central features of Generalized Anxiety Disorder (GAD) (American Psychiatric Association, 2013), a highly prevalent, chronic, and difficult to treat condition (Tyrer \& Baldwin, 2006). With a lifetime prevalence of 5.7\% (Kessler et al., 2005), GAD is associated with high rates of comorbidity with depression and with other anxiety disorders (Kessler, Chiu, Demler, Merikangas, \& Walters, 2005), increased risk of heart disease (Martens, De Jonge, Cohen, Lett \& Whooley, 2010), decreased work productivity (Henning, Turk, Mennin, Fresco \& Heimberg, 2007), and significant impairments in social and occupational functioning, at levels equivalent to individuals with chronic medical conditions (Alonso et al., 2011). Theories of GAD propose that individuals also experience a range of cognitive and affective difficulties, including, for example, cognitive avoidance (Borkovec, Alcaine \& Behar, 2004), a negative problem orientation (Robichaud and Dugas, 2005), emotion regulation difficulties (Mennin, Heimberg, Turk, \& Fresco, 2005), and difficulties tolerating uncertainty (Dugas, Gagnon, Ladouceur, \& Freeston, 1998). Theories explaining how worry leads to these difficulties are summarized below.

\section{Theories of GAD}

Worry has been theorized as a verbal-linguistic, mental activity that focuses on negative future events (Borkovec, 1985; Borkovec et al., 2004). When individuals with GAD are asked to worry as they normally do-- in words and questions (e.g., "what if I fail my exam?"), they report lower physiological arousal than when they are asked to "worry" in a form of mentation that is antithetical to verbal thinking (i.e., invoking clear vivid images of failing an exam) (Stöber, 2000). Borkovec and colleagues' (2004) cognitive avoidance theory of worry states that 
individuals engage in verbal worry to avoid thinking about feared situations in a clear and vivid way (Borkovec et al., 2004). According to the theory, verbal worry is an avoidance response that dampens fear-provoking imagery of catastrophic scenarios. Although avoiding frightening thoughts prevents individuals from experiencing excessive anxiety and other uncomfortable emotions (e.g., intense sadness) in the short-term, in the long-term, it leads to worry being negatively reinforced because individuals fail to confront and process fear. In fact, worrying in verbal form in particular increases the frequency of negative intrusions (Hirsch and Mathews, 2012).

The cognitive avoidance model has served as a foundation for subsequent theories of GAD that elaborate on the avoidant function of worry. The Intolerance of Uncertainty Model (Dugas, Buhr, \& Ladouceur, 2004; Dugas, et al., 1998; Dugas, Letarte, Rhéaume, Freeston, \& Ladouceur, 1995; Freeston, Rhéaume, Letarte, Dugas \& Ladouceur, 1994) suggests that worry facilitates not only avoidance of mental images of feared situations, but also, an intolerance of uncertainty (i.e., difficulty withstanding uncertain outcomes, and [possibly] withstanding feelings of uncertainty). According to the model, intolerance of uncertainty is a fundamental cognitive process in the development and maintenance of GAD. Individuals who are intolerant of uncertainty believe that worry will help them cope with or prevent feared events (Dugas \& Koerner, 2005). The worry in turn leads individuals to rely on a set of cognitive strategies (e.g., thought replacement, distraction, thought suppression) to facilitate avoidance of mental images of unpleasant or threatening future scenarios. According to this model, individuals also have a negative problem orientation, characterized by a lack of confidence in problem solving ability, a tendency to appraise problems as threats, frustration when dealing with problems, and pessimism about the outcome of problem-solving efforts (Dugas et al., 1998; Koerner \& Dugas, 2006; 
Robichaud \& Dugas, 2005). Together, these processes initiate and maintain episodes of worry. Worry is also associated with maladaptive perceptions of feared scenarios, including perceived likelihood, cost, and ability to cope (Berenbaum, Thompson, \& Pomerantz, 2007). Specifically, research has shown that individuals who believe that feared scenarios are more likely to occur have higher levels of worry than those who believe that feared outcomes are less likely to occur (Berenbaum et al., 2007; MacLeod, Williams, \& Bekerian, 1991). Additionally, individuals who believe that perceived undesirable outcomes will be more costly tend to have higher levels of worry (Berenbaum et al., 2007; Butler \& Mathews, 1983). Finally, low perceived ability to cope with feared outcomes is thought to be associated with worry and anxiety (Beck, 1976).

The Emotion Dysregulation Model (Mennin et al., 2005; Newman, Castonguay, Borkovec, \& Molnar, 2004; Roemer, Salters, Raffa \& Orsillo, 2005) proposes that worry enables short-term emotional avoidance but consequently dysregulates emotion. According to this model, individuals with GAD experience heightened emotional intensity, show poor emotional understanding, are strongly reactive to negative emotions (i.e., they fear their emotions, particularly anxiety), and engage in maladaptive attempts at managing emotions (Mennin, Turk, Heimberg, \& Carmin, 2004; Mennin et al., 2005). In line with this, individuals with GAD also report experiential avoidance, which refers to efforts to avoid or escape distressing thoughts, feelings and sensations (Hayes, Wilson, Gifford, Follette, \& Strosahl, 1996). From this perspective, worrying is an attempt to avoid or suppress intense negative emotions (Decker, Turk, Hess, \& Murray, 2008).

Although the cognitive and affective processes thought to underlie and maintain GAD appear on the surface to differ across the cognitive avoidance, intolerance of uncertainty and emotion dysregulation models of GAD, it has been noted that all three theories share an 
emphasis on avoidance of internal experiences: imagery of frightening, catastrophic situations, uncertainty, and negative emotions, respectively. Therefore, all evidence-based psychological treatments for GAD include strategies to address avoidance. Given that many patients obtain only partial benefit from existing treatments, GAD remains one of the least successfully treated anxiety disorders (Gould \& Safren, 2004; Hanrahan, Field, Jones \& Davey, 2013; Waters \& Craske, 2005), suggesting that treatment techniques need refining. A promising direction for improving treatment efficacy is closer examination of individual components of psychological treatment packages for GAD.

\section{Treatment for GAD}

It is proposed that one treatment strategy warranting closer investigation is imaginal exposure, a key component of several CBT protocols for GAD (Borkovec \& Costello, 1993; Mennin, 2004; Robichaud \& Dugas, 2006). Imaginal exposure for GAD has been examined independently of other CBT techniques in only a small number of studies (Hoyer et al., 2009; Provencher, Dugas, \& Ladouceur, 2004; Shahmoradi et al., 2013).

Imaginal exposure, when delivered within the context of a multicomponent CBT for GAD, is based on the notion that pathological worry is caused in part by a tendency to avoid thinking about feared situations in a clear way (Borkovec et al., 2004). In imaginal exposure for GAD, individuals are instructed to conjure up a vivid mental image of their feared worst-case scenario coming true. The story narrative is written in the first person, and in the present tense (Dugas \& Koerner, 2005; Dugas \& Robichaud, 2007). The goals of this form of exposure are to help individuals counteract their avoidance by having them fully imagine and process concrete and vivid images of their worst fear coming true, instead of worrying to reduce emotional response (Dugas \& Koerner, 2005). A common way to deliver imaginal exposure for GAD is to 
have people audiorecord themselves "telling a story" about their worst fear coming true and listening to the recording for 20 to 60 minutes every day. Eight to fifteen sessions of imaginal exposure delivered in this manner leads to large, significant improvements in worry and GAD symptoms (Hoyer et al., 2009; Provencher et al., 2004; Shahmoradi et al., 2013).

Another way in which imaginal exposure for GAD has been delivered is as a structured writing procedure (termed written exposure; Goldman, Dugas, Sexton, \& Gervais, 2007; Robichaud \& Dugas, 2015). In written exposure for GAD, individuals are instructed to write a detailed description of their worst fear coming true, as if it is happening in the here and now, with reference to their emotions, physical sensations, and reactions to the worst fear.

Goldman et al. (2007) compared the impact of written exposure to that of neutral writing across five 30-minute sessions in a sample of individuals high in the tendency to worry. Participants in the written exposure condition were instructed to write about the same feared situation on 5 consecutive days but were told that they could go deeper into their scenario with each successive session. Individuals assigned to the neutral condition were asked on five consecutive days to write an unemotional story describing what they would do if they found out that they had the day off work. Participants in the written exposure condition showed significant improvements in worry, from baseline to 2-week follow-up $(d=1.22)$, whereas participants who engaged in neutral writing did not $(d=0.45)$. However, there were significant between-group differences at 2-week follow-up neither on worry $(d=0.40)$ nor on GAD-associated symptoms $(d=0.22)$. Fracalanza, Koerner and Antony (2014) examined the effects of three, 20-minute sessions of written exposure on GAD symptoms. On each of 3 days, they asked participants to (a) write about the same worst-case scenario as per Goldman et al. (2007), (b) write about a different worst-case scenario related to the same worry theme, or (c) write about a neutral 
scenario. Writing repeatedly about the same worst-case scenario produced large significant decreases in worry from baseline to 1-week follow-up, $(d=0.91)$, whereas writing about a different worst-case or a neutral scenario $\operatorname{did} \operatorname{not}(d=0.04$ and $d=0.35$, respectively).

Individuals who wrote repeatedly about the same worst-case scenario also demonstrated a large significant decrease in attempts to avoid imagining their worst fear coming true $(d=0.97)$, whereas this effect was not seen in individuals who wrote about a different worst-case scenario or a neutral scenario at each session $(d=0.29$ and $d=0.29)$. Participants who wrote repeatedly about the same worst-case scenario also displayed a significant decrease in intolerance of uncertainty from baseline to 1-week follow-up, $(d=0.70)$, whereas those writing about different worst-case scenarios or the same neutral scenario did not $(d=0.40, d<0.01)$. Despite significant within-group differences, there were no between-group differences on these outcomes at followup. Nonetheless, the findings in Fracalanza and colleagues (2014) study are interesting, particularly regarding intolerance of uncertainty. Even though written exposure is not explicitly designed to modify negative beliefs about uncertainty, it seems to improve these beliefs anyway. According to the IUM, imaginal exposure targets cognitive avoidance, but perhaps also has therapeutic effects on other processes such as intolerance of uncertainty, because individuals learn to challenge the meanings given to uncertain future events (Dugas \& Koerner, 2005).

While the few studies examining written exposure as a standalone treatment for GAD are encouraging, little is known on how to best conduct the procedure. For example, what should the exposure target be? Many treatment manuals for GAD recommend imaginal exposure to the worst worry coming true; but there actually is not a compelling empirical basis for this, aside from Borkovec's early theorizing of worry as "cognitive avoidance" of feared hypothetical situations. In general, there is a serious dearth of research on how to do exposure with people 
who have GAD, even though treatment manuals usually include exposure as one of the recommended strategies (e.g., Craske, Barlow \& O’Leary, 1992; Dugas \& Robichaud, 2007). However, many individuals with GAD do not respond to the best treatments, and continue to struggle with the chronic and disabling course of the disorder. Thus, it is imperative to make improvements to existing treatments.

Based on existing theories of GAD, exposure could be enhanced by incorporating strategies to address the range of cognitive difficulties that individuals with GAD experience. Perhaps current exposure procedures for GAD are not optimized to directly reduce avoidance and change maladaptive cognitions. I propose that one possible way to enhance written exposure for GAD is by adding a "rescripting" component to the procedure that is designed to modify unhelpful beliefs. In therapy, rescripting essentially involves rewriting a personal narrative so that the outcome is not so unpleasant.

\section{Rescripting}

There is no existing literature on a rescripting intervention for GAD, but the literature on imagery rescripting offers some direction as to how rescripting could augment the efficacy of written exposure for chronic worry. Imagery rescripting (IR) refers to a set of imagery techniques aimed at changing the negative meanings associated with memories of traumatic or distressing experiences. In Smucker and colleagues' (1995) original three-step protocol, individuals are first asked to vividly recall and recount a distressing traumatic memory in the present tense (imaginal exposure). Next, in the rescripting phase, they are asked to reimagine the event and to intervene, in the imagination, to gain a sense of mastery over the distressing mental images. Finally, individuals are asked to develop images of their current adult-self comforting their past-self. These steps are intended to challenge and modify negative thoughts, feelings, and 
behaviours, or promote new, positive images to counteract key psychological concerns (Holmes, Arntz \& Smucker, 2007). IR, applied across several psychological disorders, shares the central notion that mental images are associated with more emotion than are verbal thoughts (Holmes \& Mathews, 2005). Thus, from a treatment perspective, the most effective way to modify imagery is by using techniques involving imagery (Holmes et al., 2007). What sets apart IR from other imagery modification treatments, such as imaginal exposure, is that individuals create their own changes to images, accomplished through a Socratic style of questioning that encourages reflection and problem solving (Rusch, Grunert, Mendelsohn \& Smucker, 2000). Despite being promising, IR has not been used in the treatment of GAD, but has been adapted and successfully applied across a range of other disorders (e.g., PTSD: Grunnert, Smucker, Weis, \& Rusch, 2003; social anxiety disorder: Wild, Hackman, \& Clark, 2007; eating disorders: Cooper, 2011; snake phobia: Hunt \& Fenton, 2007; personality disorders: Weertman \& Arntz, 2007; depression: Wheatley \& Hackmann, 2011). Data on efficacy of IR are summarized below.

\section{Efficacy of IR}

A handful of studies have explored the efficacy of IR compared to or combined with other interventions. For instance, Arntz, Kindt and Tiesema (2007) compared the effectiveness of imaginal exposure (IE) to the combination of imaginal exposure with imagery rescripting (IE+IR) in a PTSD sample. At 1-week posttreatment, participants in the IE+IR group showed significant improvements in anger control $(d=0.61)$, hostility $(d=0.53)$, guilt $(d=0.96)$ and shame $(d=0.43)$, whereas participants in the IE group did not $(d=0.13, d=0.19, d=0.25, d=-$ 0.06). Furthermore, fewer patients dropped out of the IE + IR treatment than out of the IE treatment (25\% vs. 51\%). In line with this, Grunert, Weis, Smucker and Christianson (2007) found that PTSD patients who had failed to improve from an imaginal exposure intervention 
benefited from 1 to 3 sessions of imagery rescripting. After the IR intervention, individuals showed significant improvements in avoidance $(d=-1.35)$, intrusive symptomatology $(d=-$ $1.92)$, depression $(d=-1.15)$, state anxiety $(d=-0.94)$ and trait anxiety $(d=-0.58)$.

Wild, Hackman and Clark (2007) were the first to test the effects of a single 90-minute session of IR in individuals with social phobia. From baseline to 1-week follow-up, the researchers found strong effects on beliefs associated with the distorted images of the self and beliefs associated with distressing memories (encapsulated beliefs) $(d=2.51)$, image distress $(d$ $=1.44)$ and memory distress $(d=1.79)$. In addition, 1 week after the session, there were significant improvements in social phobia indices, including frequency of negative social cognitions $(d=1.03)$ and encapsulated beliefs $(d=1.49)$. In a subsequent study, Wild, Hackmann and Clark (2008) compared one session of imagery rescripting to one control session of exploration of early memories. No change was observed after the control session, but from baseline to 1-week follow up, the single IR session led to significant, large improvements in negative beliefs about the meaning of the traumatic memory $(d=2.92)$, the distress associated with the memory $(d=1.32)$, distress associated with negative images $(d=1.05)$, anxiety in feared social situations $(d=0.90)$ and fear of negative evaluation $(d=0.72)$.

Hagenaarz and Arntz (2012) studied the effects of IR compared to positive imagery or imaginal exposure after an analogue trauma, in healthy volunteers. All participants watched an aversive film, had a 30-minute break, and then received their assigned intervention. Participants assigned to the IR condition were asked to recall and re-experience a scene from the film for the first 3 minutes and then change whatever they did not like in the scene for a more pleasing outcome. Participants assigned to the IE condition were asked to recall and re-experience a scene from the film in as much detail as possible for 9 minutes and received no further instructions to 
modify their mental image of the scene. Those assigned to the positive imagery condition were asked to select a personal, pleasant experience and to recall and re-experience that. Posthoc comparisons indicated that participants in the IR condition experienced fewer intrusive memories of the scene than did those in the positive imagery or IE conditions $(p=.047, d=.57$ and $p=.004, d=.87$, respectively). Posthoc comparisons showed that scores on a measure of posttraumatic cognitions were lower in the IR and positive imagery conditions compared to the IE condition ( $p=.02, d=.68$ and $p=.03, d=.63$, respectively). In addition, people assigned to IR reported less negative views of the world at the end of the intervention compared to participants assigned to IE ( $p=.005, d=.85$ ) or positive imagery $(p=.09, d=.49)$. Selfblame was also lower in the IR condition ( $p=.04, d=.62)$ and in the positive imagery condition $(p=.007, d=.78)$ than in the IE condition.

Finally, a study by Long and colleagues (2011) is the only known study that has attempted to examine mechanisms of change in IR. The researchers found that change in total PTSD-related negative cognitions (beliefs about the self, world, and self-blame) was significantly correlated with change in PTSD symptoms $(r=.49, p=.017)$. Although determining the direction of causality requires further research, the findings support the idea that reductions in trauma-related cognitions are associated with reductions in PTSD symptoms, and provide preliminary evidence that one of the mechanisms of change of IR may be the modification of maladaptive beliefs.

On the whole, these studies suggest that IR seems to have better effects on nonfearrelated emotions, such as anger, shame or guilt, than does imaginal exposure. The studies also suggest that IR may better address core beliefs and disorder-related cognitions than imaginal exposure. Thus, in the treatment of GAD, enhancing exposure with rescripting may also target a 
broader range of worry-related processes compared with exposure alone.

\section{Enhancing Exposure for GAD with Rescripting}

IR has not been used in the treatment of GAD, perhaps because it remains unclear how it can be adapted for this population. For example, if worry is a verbal-linguistic activity, and if it remains unclear what the underlying negative image or feared outcome is in individuals with GAD, can IR, in theory, still be successfully applied to this population? The personality disorders research has shown that the use of imagery to introduce positive or benign meanings helps counteract negative schematic beliefs, even if these beliefs are not in the form of an image (Arntz \& Weertman, 1999; van Tilburg et al., 2006 ; Weertman \& Arntz, 2007 ; Young, Klosko, \& Weishaar, 2003). Furthermore, research on IR has also shown that it is still possible to promote new or positive images, even if there is no distressing, underlying negative image (Holmes et al., 2007). Questions also may arise regarding applicability of IR for scenarios that have not yet occurred. Yet research suggests that a common neural network underlies both memory and imagination (Holmes \& Matthews, 2010; Schacter, 2012). Therefore, even though the application of IR to GAD may not seem indicated, the evidence suggests otherwise.

\section{Proposed steps of rescripting for GAD.}

Given that individuals with GAD avoid thinking about feared situations in a clear way, an important first step in a rescripting protocol for GAD would be written exposure to the worstcase scenario, wherein individuals would be asked to write concretely about their worst fear coming true. Concrete sentences activate more emotionally charged imagery than do abstract words and sentences (Paivio \& Marschark, 1991), which in turn encourages emotional processing (Stöber, 1998; 2000). Thus, asking individuals to write concretely would allow them to counteract their avoidance by fully experiencing and processing clear, vivid images of their 
worst fear coming true. Following the written exposure phase would be the rescripting phase, which should be closely related to key disorder-specific cognitive and emotional problems in order to be meaningful, because changing responses to threatening stimuli requires changing maladaptive cognitive beliefs (Wheatley \& Hackmann, 2011). The assumption as to why rescripting is likely to be an effective enhancement for exposure in the treatment of GAD is embedded in a cognitive rationale. A rescripting intervention for GAD would require individuals to imagine altering their response to their feared worst-case scenario in ways that challenge their maladaptive beliefs about future hypothetical events and their capacity to competently deal with these events. Therefore for individuals with GAD, the rescripting phase should be aimed at challenging GAD-specific maladaptive cognitions, such as beliefs about uncertainty, negative problem orientation, and perceptions of consequences of anxiety (i.e., the fear of anxiety). It is proposed that for individuals with GAD, it would be insufficient to simply ask individuals to imagine a highly positive outcome that could never happen or is highly unlikely, because it would not provide the corrective information needed to challenge the maladaptive cognitions observed in individuals with GAD. For individuals with GAD then, the goal would not be to alter the worst-case scenario into the best-case scenario, but rather to alter their beliefs about how they would manage the worst-case scenario if it did occur (i.e., modifying the response elements of the image; see Lang, Levin, Miller \& Kozak, 1983). Taken together, the written exposure phase would expose individuals to concrete thoughts of the worst-case scenario and activate images, emotions and beliefs associated with the feared worst-case scenario and the rescripting phase would go a step further and require individuals to concretely imagine responding to this worstcase scenario in an adaptive way. Research suggests that thinking about problems concretely (versus abstractly) is adaptive because it enables individuals to prepare for, prevent, or solve 
negative consequences of problems (Stöber \& Brokovec, 2002; Watkins, 2004). In addition, evidence suggests that training people to think more concretely about situations not only promotes more effective solutions to interpersonal problems (Pawluk, Koerner, \& Antony, 2011), but also reduces emotional reactivity to stressors (Watkins, Moberly, \& Moulds, 2008). Therefore, keeping the rescripting phase as concrete as possible seems essential.

\section{Present Study: Research Questions and Hypotheses}

The present study tested whether a written exposure procedure that includes a rescripting component (from hereon, "written exposure with rescripting") is better at improving GAD symptoms and maladaptive cognitive processes compared to a written exposure procedure that does not include this component (from hereon, "worst-case scenario exposure"). The current study extends what is known about exposure for individuals with GAD by comparing the effects of three sessions of written exposure with rescripting (RWE) to those of three sessions of the conventional procedure (written exposure to the worst-case scenario, WE) or neutral writing.

The first objective was to examine whether written exposure enhanced with rescripting is more efficacious in reducing worry and GAD symptoms than repeated written exposure to the same worst-case scenario without rescripting or repeated sessions of neutral writing. In addition, given that high levels of anxiety and depression can often accompany excessive worry (Hirsch, Mathews, Lequertier, Perman \& Hayes, 2013), improvements in these symptoms were also examined. It was hypothesized that participants in the RWE and WE conditions would show greater decreases in worry, GAD symptoms, as well as anxiety and depressive symptoms, compared to participants in the neutral condition, but that the greatest decreases would be for participants assigned to RWE. 
The second objective was to examine the degree to which GAD-related cognitive processes improve following each of the exposure interventions. These processes included intolerance of uncertainty, cognitive avoidance, a negative problem orientation, negative beliefs about emotions and emotional arousal, inflated perceptions about the likelihood and cost associated with one's worst fear, and negative beliefs about one's ability to cope with the worst fear. It was hypothesized that participants in the RWE and WE conditions would show greater improvements in GAD-related cognitive processes compared to neutral writing, but that RWE would lead to the greatest improvements.

The third objective was to examine how the written exposure interventions result in reductions in worry, the central feature of GAD. It was hypothesized that changes in intolerance of uncertainty and a negative problem orientation would mediate changes in worry in RWE and WE, albeit to a greater extent in the RWE condition. 


\section{Method}

\section{Participants}

$N=265$ adults were recruited through online and print advertisements, and from a database of participants who participated in other studies in our lab. Individuals were initially screened over the phone. The phone screen included questions to assess the potential presence of pathological worry and GAD symptoms, gateway questions from the Mini International Neuropsychiatric Interview (MINI), questions to rule out suicidality, as well as the Penn State Worry Questionnaire (PSWQ). Of the 265 individuals who participated in the phone screen, 95 met inclusion criteria (as defined below) and were invited to the lab. Primary reasons for exclusion included subclinical levels of worry, and symptoms consistent with another diagnosis (e.g., social anxiety disorder, depression) that was more severe than the individual's GAD. Ten eligible individuals did not begin the study, four individuals were not eligible after the in-person MINI interview, and 17 did not complete all study visits (seven dropped out after visit 1, one dropped out after visit 2, one dropped out before the 1-week follow-up, and eight dropped out at 1-month follow-up). Individuals who dropped out without completing all writing sessions were excluded from analyses. This was done to ensure that participants in all conditions received the same "dose" of writing, as well as the intended "dose" of the interventions. Data screening (see below) eliminated an additional six participants; (four for not adhering to writing instructions;

one for scoring below 62 on the PSWQ - incorrectly scored during the phone screening; and one participant aged 70). The final sample comprised 67 adults. Excluded participants were not significantly different from the rest of the sample on any demographic characteristics or other baseline measure scores.

Inclusion criteria included: (1) age between 18 and 65 years; (2) endorsement of 
symptoms consistent with a principal diagnosis of DSM-5 GAD (American Psychiatric Association, 2013); (3) the presence of excessive worry as indicated by a PSWQ score of 62 or above (Behar, Alcaine, Zuellig, \& Borkovec, 2003), a cut-score that provides an optimal balance of sensitivity and specificity and effectively discriminates GAD from PTSD and depression (Behar et al., 2003); (4) stable psychotropic medication dosage (if taking medication) for at least 6 weeks prior to study entry or, if discontinued medication, medication-free for 1 month or 3 months for selective serotonin reuptake inhibitors (e.g., fluoxetine); (5) no current endorsement of bipolar and related disorders or a psychotic disorder; (6) early or sustained remission in the case of substance use disorder; (7) no endorsement of current, serious suicidal ideation, intent, or plan; (8) no psychotherapy in the past 3 months.

\section{Demographic Characteristics}

The final sample was composed of 56 females and 11 males, with ages ranging from 18 to 46 years ( $M=26.07$ years; $S D=6.11$ years). More than half the sample $(62 \%)$ reported being single, $15.2 \%$ reported being married/common law, and $5.1 \%$ reported being divorced or widowed. In terms of race/ethnicity, the breakdown was as follows: White (34.2\%), East Asian (8.9\%), South Asian (7.6\%), Mixed (7.6\%), South East Asian (6.3\%), Latin American (6.3\%), Other (5.1\%), Black (3.8\%), Arab/West Asian (2.5\%). In terms of highest education achieved, $19 \%$ of participants reported having completed an undergraduate degree; $10.1 \%$ reported completing a Doctoral degree, $8.9 \%$ reported completing a Master's degree, $2.5 \%$ reported completing a college degree and $2.5 \%$ reported completing high school.

\section{Clinical Characteristics}

Individuals with comorbid diagnoses were included in the study provided GAD was rated at least 2 points more severe on the Clinician's Severity Rating scale from the Anxiety Disorders 
Interview Schedule for DSM-IV (ADIS-IV; Di Nardo, Brown, \& Barlow, 1994). Over half the sample (69.2\%) met diagnostic criteria for GAD only, $10.8 \%$ had a comorbid mood disorder, 15.4\% had a comorbid anxiety disorder, and $4.6 \%$ had another comorbid disorder. PSWQ scores for the current sample were well above the established cut-score of $62(M=69.29, S D=4.93)$, with $79.1 \%$ of the sample scoring above the more stringent cut-score of 65 (Fresco et al., 2003), and GAD-Q-IV mean scores were above 7.67, indicative of the presence of likely GAD $(M=$ 9.48, $S D=2.43$ ). Table 1 contains a summary of demographic and clinical characteristics of participants. There were no significant differences between study conditions in any of these characteristics.

\section{Measures}

The Mini International Neuropsychiatric Interview (MINI) Screen, Version 5.0.0

(Sheehan et al., 1998) is a brief preliminary screening tool for the Mini International Neuropsychiatric Interview. It consists of 21 closed-ended screening questions about current mood and anxiety disorder symptoms, suicidality, substance use and eating disorders. Positive responses to screening questions mean that the interviewer should conduct the corresponding module in the MINI to assess related symptoms in more detail. This version of the MINI Screen (for Axis-I DSM-IV disorders) has been shown to have high internal consistency ( $\alpha=.92$;

Sheehan et al., 1998). The screening questions have ranged from $61 \%$ to $83 \%$ on specificity and the accuracy of the questions has ranged from 70\% to 75\% (Alexander, Haugland, Lin, Bertollo, $\&$ McCorry, 2008).

The Mini International Neuropsychiatric Interview, Version 7.0.0 (MINI; Sheehan et al., 2015) is a brief semistructured diagnostic interview that assesses the presence of certain DSM-5 disorders. Given the recent release of the MINI 7.0, psychometric properties are not yet 
available, however, the MINI for Axis-I DSM-IV disorders has shown excellent interrater reliability, with kappa coefficients that range from .88 to 1.0 , good test-retest reliability for GAD, $r=.78$ to $r=.93$ (Lecrubier et al., 1997; Sheehan et al., 1998), and good specificity (86\%) and sensitivity (91\%) for all diagnoses (Sheehan et al., 1998). The MINI for Axis-I DSM-IV disorders also has shown high convergent validity in relation to other semistructured clinical interviews, such as the Structured Clinical Interview for the Diagnostic and Statistical Manual (SCID; Lecrubier et al., 1997; Sheehan et al., 1998).

The Penn State Worry Questionnaire (PSWQ; Meyer, Miller, Metzger, \& Borkovec, 1990) is a 16-item self-report measure that assesses a general tendency to worry excessively. Items are rated on a 5-point Likert scale, ranging from 1 "not at all typical of me" to 5 "very typical of me." Total scores range from 16 to 80, with higher scores reflecting greater levels of pathological worry. The PSWQ has demonstrated very high internal consistency $(\alpha=.88$ to .95$)$ (Molina \& Borkovec, 1994), and good test-retest reliability ( $r=.92$; Metzger, Miller, Cohen, Sofka, \& Borkovec, 1990). Previous research using a PSWQ cutoff score of 62 in an analogue GAD sample showed good sensitivity (75\%) and specificity (86\%) for detecting individuals with GAD (Behar et al., 2003).

The Penn State Worry Questionnaire-Past Week (PSWQ-PW) (Stöber \& Bittencourt, 1998) is an adaptation of the original PSWQ (Meyer et al., 1990) intended to capture pathological worry during the past week. The PSWQ-PW is thus sensitive to change and can be used to assess changes in pathological worry during an intervention (Stöber 2002; Stöber \& Bittencourt, 1998). The PSWQ-PW has demonstrated excellent internal consistency $(\alpha=.91)$ and good test-retest reliability $(r=.59)$, and good content and construct validity (Stöber \& Bittencourt, 1998). 
The Generalized Anxiety Disorder Questionnaire for the DSM-IV (GAD-Q-IV; Newman et al., 2002) is a 9-item self-report measure that assesses the DSM-IV diagnostic criteria for GAD. Given that the core diagnostic criteria for GAD remain unchanged in DSM-5, this tool is suitable to assess symptoms of DSM-5 GAD. A cutoff of 7.67 (85\% sensitivity and 74\% specificity) on the GAD-Q-IV indicates the presence of likely GAD (Moore, Anderson, Barnes, Haigh, \& Fresco, 2014). The GAD-Q-IV total score has good test-retest reliability $(r=.83)$ (Newman et al., 2002), high convergent validity with other measures of GAD features, and discriminant validity when compared against measures of depression (Robinson, Klenck, \& Norton, 2010).

The Intolerance of Uncertainty Scale (IUS; Freeston et al., 1994; English translation, Buhr \& Dugas, 2002) is a 27-item self-report measure that assesses negative beliefs about uncertainty. Items are rated on a 5-point Likert scale, ranging from 1 "not at all characteristic of me" to 5 "very characteristic of me." Total scores range from 27 to 135, with higher scores indicating a general tendency or predisposition to see uncertainty as unacceptable (Buhr \& Dugas, 2002). The IUS has demonstrated excellent internal consistency $(\alpha=.95)$, good test-retest reliability $(r=.92)$ (Sexton \& Dugas, 2009), and high convergent, criterion, and discriminant validity (Buhr \& Dugas, 2002).

The Cognitive Avoidance Questionnaire (CAQ; Sexton \& Dugas, 2008) is a 25- item self-report measure that assesses the tendency to engage in five cognitive avoidance strategies: thought substitution, transformation of images to verbal thoughts, distraction, avoidance of stimuli that trigger unpleasant thoughts, and thought suppression. Items are rated on a Likert scale ranging from 1 "not at all typical" to 5 "completely typical," respondents endorse how true each statement is of them. Scores range from 25 to 125, with higher scores indicating a greater 
tendency to cognitively avoid threatening internal events. The CAQ demonstrates excellent internal consistency $(\alpha=.95)$, good test-retest reliability $(r=.85)$ and good convergent and divergent validity (Sexton \& Dugas, 2008).

The Negative Problem Orientation Questionnaire (NPOQ; Gosselin, Pelletier, \& Ladouceur, 2001; Robichaud \& Dugas, 2005) is a 12-item self-report measure that assesses the tendency to view problems as a threat, doubt one's own problem solving ability, and be pessimistic about the outcome of a problem. Respondents rate items on a 5-point Likert scale ranging from 1 "not at all true of me" to 5 "very true of me," according to how they react or think when confronted with a problem. Scores range from 12 to 60 , with higher scores indicating greater negative attitudes towards problems. The NPOQ has high internal consistency $(\alpha=.91)$ good test-retest reliability $(r=.80)$, and good convergent and discriminant validity (Robichaud \& Dugas, 2005).

The Affective Control Scale (ACS; Williams, Chambless, \& Ahrens, 1997) is a 42-item self-report measure that assesses fear of emotions and attempts to control emotional experience. Items are scored on a 7-point Likert scale ranging from 1 "very strongly disagree" to 7 " very strongly agree." The ACS contains four subscales: 1) Fear of anxiety; 2) Fear of depression; 3) Fear of anger; 4) Fear of positive emotions. The subscales have demonstrated good test-retest reliability $(r=.78)$, and good internal consistency (Berg, Shapiro, Chambless, \& Ahrens, 1998; Williams et al., 1997). For the present study, the total scale score and the "fear of anxiety" subscale were analyzed. Total scores range from 42 to 294; subscale scores range from 13 to 91 , with higher scores indicating a greater fear of anxiety.

Perceived Probability, Cost, and Coping Questions (Berenbaum et al., 2007; Butler \& Mathews, 1983) were administered to assess the perceived probability, perceived cost, and 
perceived ability to cope with the worst-case scenario, given that these variables have previously been identified as being important indicators of cognitive change (e.g., Berenbaum et al., 2007; Fracalanza et al., 2014). As per Fracalanza (2010), perceived probability was assessed by asking respondents to rate the likelihood that their worst fear (identified via the catastrophizing interview) will come true on a 7-point Likert scale, ranging from 0 "not at all likely" to 6 "almost certain." Perceived cost was assessed by asking respondents to rate how bad it would be if their identified worst fear came true, from 0 "not at all bad" to 6 "horrific." Perceived ability to cope was assessed by asking respondents to rate the extent to which they would be able to cope if their identified worst fear came true, from 0 "not at all" to 6 "would be able to cope."

The State-Trait Inventory for Cognitive and Somatic Anxiety (STICSA; Ree, French, MacLeod, \& Locke, 2000) comprises two scales designed to assess cognitive and somatic symptoms of anxiety as they pertain to an individual's current state (State scale, STICSA-S) and their general disposition (Trait scale, STICSA-T). Each scale consists of the same 21 items. On the STICSA-S, respondents rate each item on a 4-point Likert scale, ranging from 1 "not at all" to 4 "very much so." On the STICSA-T, respondents rate each item from 1 "almost never" to 4 "almost always." Scores on each scale range from 21 to 84, with greater scores indicating greater cognitive or somatic trait anxiety. Both scales have demonstrated good test-retest reliability $(r$ $=.70$ and $r=.88$, respectively), high internal consistency $(\alpha=.91)$ and good convergent and discriminant validity (Grös, Antony, Simms, \& McCabe, 2007).

The Center for Epidemiologic Studies Depression Scale—Revised (CESD-R; Eaton, Muntaner, Smith, Tien, \& Ybarra, 2004) is an updated version of the Center for Epidemiologic Studies Depression Scale (Radloff, 1977). The CESD-R consists of 20 items that more closely reflect DSM-IV depression criteria than did the original CES-D. Respondents rate the degree to 
which they experienced depressive symptoms over the past week. Ratings are based on a 5-point Likert scale ranging from 0 "not at all or less than 1 day" to 4 "nearly every day for 2 weeks." The CESD-R has good psychometric properties, including high internal consistency $(\alpha=0.93)$, good test-retest reliability $(r=.70)$ and good convergent and discriminant validity (Eaton et al., 2004; Van Dam \& Earleywine, 2011). Given that the core symptoms of a major depressive episode (MDE) remain unchanged in DSM-5, the CESD-R is suitable for the assessment of depressive severity.

The Self-Assessment Manikin (SAM; Bradley \& Lang, 1994) is a pictorial assessment technique used to measure emotional responses along two dimensions: arousal and valence. The SAM requires respondents to circle the manikin that reflects how they are feeling, and these are coded on a 9-point scale. For the arousal dimension, SAM figures range from a figure with closed eyes and an inactive body coded as 1 "very calm" to a figure with an active body and wide eyes coded as 9 "very aroused." For the unpleasant affect dimension, SAM figures range from a smiling figure coded as 1 "very pleasant" to a frowning figure coded as 9 "very unpleasant." The SAM has been used extensively in research on emotions and has demonstrated strong correlations with physiological and behavioural measurements of pleasure and arousal (Bradley \& Lang, 1994). In addition, it is sensitive to changes in anxious arousal during writing interventions (e.g., Fracalanza et al., 2014; Sloan, Marx \& Epstein, 2005).

\section{Procedure}

Participants deemed eligible following the telephone screen were invited to the lab for an in-person MINI interview to confirm the presence of a principal diagnosis of GAD. Those found to be eligible for continued participation were asked to complete a demographics questionnaire and a baseline questionnaire package consisting of the following measures: PSWQ, PSWQ-PW, 


\section{GAD-Q-IV, IUS, CAQ, NPOQ, ACS, STICSTA-S, STICSTA-T, CES-D-R, SAM.}

Following completion of baseline measures, participants were administered the Worry Domains Rating Form (WDRF; Fracalanza et al., 2014). The WDRF was developed to assess the degree to which participants worry about several specific topics, with item ratings ranging from 0 "no worry" to 10 "extreme worry." The WDRF consists of a list of 12 worry domains derived from published research (Davey, Hampton, Farrell, \& Davidson, 1992; Dugas, Freeston et al., 1998; Freeston, Dugas, \& Ladoucer, 1996). The worry domains include friendships, romantic relationships, relationships with parents, academic performance, work competence, finances, one's own health, health of loved ones, threat of physical harm or danger, the future, selfconcept, and minor matters. The WDRF contains four blank spaces to indicate and rate worries not on this list. If more than one worry domain is assigned the same rating, respondents are asked to indicate the most bothersome one. The experimenter worked with the participant to determine the worst-case scenario by first identifying the primary worry domain on the WDRF. After identification of the primary worry domain, the experimenter worked with the participant to determine the worst-case scenario via the catastrophizing interview (Davey \& Levy, 1998; Vasey \& Borkovec, 1992). This involved asking participants "What is it that worries you about (X)?" For example, if the participant's main worry on the WDRF was academic performance, the first question would be "What is it that worries you about your academic performance?" until the worst-case scenario was identified (as per Fracalanza et al., 2014). For an example of the catastrophizing interview, see figure 1. Following identification of the worst-case scenario, participants were asked several questions to make the account of the feared scenario more concrete (e.g. "What does the scenario look like?", "Where are you?" "What do you see?" Next, participants were administered Perceived Probability, Cost, and Coping Questions pertaining to 
the identified worst-case scenario.

Next, participants were randomly assigned to one of three conditions: (1) Worst-case scenario exposure (WE), (2) Written exposure with rescripting (RWE) or (3) Neutral Control (NC). Participants were informed that they would be asked to write on 3 consecutive days for 30 minutes each day. A meta-analysis on written disclosure, the writing intervention that partly informed the development of written exposure (see Goldman et al., 2007), has shown that three sessions of writing demonstrates greater effect sizes compared to studies that use less than three sessions (Frattaroli, 2006). In addition, previous research has shown that writing for periods shorter than 15 minutes is associated with smaller effects sizes than studies in which participants write for at least 15 minutes (Frattaroli, 2006). All participants were informed that the purpose of the study was to examine the relationship between worry and writing (Appendix A). All writing occurred alone, in a private room. On each day, all participants were read the instructions about the writing that they would be asked to do and were given a written copy of these instructions. To assess anxious arousal and unpleasant affect, participants completed the SAM just prior to the writing session, 15 minutes into the writing session and at the end of the writing session.

\section{WE Condition}

Sessions 1, 2 and 3. Individuals assigned to the WE condition were asked to write on 3 consecutive days, a story (sensory image "script") describing their worst fear (identified via the catastrophizing interview) coming true (Appendix B). Specifically, participants were instructed to: (1) write in narrative form, beginning with a description of the circumstances leading up to the feared scenario, followed by a description of the actual feared scenario, and ending with a description of the consequences of the scenario; (2) write in the present tense as though the situation was happening in the here-and-now; and (3) describe in detail, their emotional and 
physical reactions to the scenario (e.g., "I am scared," "I am nauseous").

\section{RWE Condition}

Sessions 1, 2 and 3. On day 1, participants assigned to the RWE condition were asked to write a sensory image script describing their worst fear coming true (as per the WE condition).

On day 2, participants were asked to write a sensory image script describing their worst fear coming true, and to write about how they could move forward to improve the situation and how they could change their attitude about the situation. On day 3, participants were asked again to write about how they could move forward to improve the situation or change their attitude about the situation (Appendix C). Participants were asked to write in first-person, present tense, as though they were responding to the situation in the here-and-now, with details regarding their emotional and physical reactions.

\section{NC Condition}

Sessions 1, 2 and 3. Individuals assigned to the NC condition were asked to write on days 1 to 3 about what they would do if they found out they had the day off. They were asked to write in a completely factual way, with no reference to emotions or opinions (see Appendix D). The neutral condition was employed to control for any therapeutic effects associated with the act of writing.

\section{Follow-up Sessions}

Outcome measures were re-administered at the end of the third writing session, and at 1week and 1-month follow ups. Participants were compensated at each visit, amounting up to a total of \$55 spread across visits. Participants were debriefed at their final visit. 


\section{Results}

\section{Data Screening}

Prior to analysis, data were screened for missing values, violations of assumptions of normality, and the presence of outliers. No outliers, as defined by values outside a $z$-score of \pm 3.29 (Tabachnick \& Fidell, 2013), were identified. Examination of histograms suggested that data were normally distributed, and skewness and kurtosis statistics indicated that the assumption of normality was met. Missing values on questionnaires were replaced by variable means, a method suitable when the rate of missing data is 5\% or less (Schafer, 1999).

\section{Data Analytic Plan}

Hypotheses 1 and 2 were tested using Hierarchical Linear Modeling (HLM; Raudenbush, Bryk, Cheong, Congdon, \& du Toit, 2004), as opposed to Analyses of Variance (ANOVA), given HLM's advantages. Specifically, HLM is particularly suitable when the data structure is nested, allowing for examination of within and between participant change across time and by condition. HLM also has less strict assumptions and more flexible data requirements (e.g., dealing with dropouts or missing data), ultimately providing more precise estimates of intervention effects. Of note, there is a great deal of variation in both HLM modeling approaches and reporting of results in the psychology literature, as evidenced by a review of studies using hierarchical linear models (Barr, Levy, Scheepers \& Tily, 2013). Specifically, the authors of this review note a lack of standards for reporting the modeling procedure and results. In line with this, the sixth edition of the APA manual does not have specific guidelines for reporting HLM modeling procedures and results.

In the current study, two sets of HLM analyses were conducted to compare change among RWE, WE, and NC conditions over time on the following outcome variables: PSWQ, 
GAD-Q-IV, STICSA-T, CESD-R, IUS, CAQ, NPOQ, ACS total and anxiety subscale scores, and perceived probability, cost, and coping ratings. First, HLM analyses were conducted to examine the main effects of Time over the total study duration (baseline to 1-month) and Condition; between group differences were assessed by examining the Time $\mathrm{X}$ Condition interaction. Separate HLM analyses were conducted for each outcome measure for each of the following comparisons: RWE vs. NC, WE vs. NC and RWE vs. WE. Modeling change over the total study duration assumes that the rate of change is consistent across the total study duration (i.e., baseline to 1-month). However, in psychotherapy research, change tends to occur in distinct phases, such as a period of rapid improvement followed by a reduced period of change. That is, it is common to see differences in rates of change between the active treatment phase and followup phase (e.g., Keller et al., 2000; Young, Kranzler, Gallop \& Mufson, 2012). This pattern of change can be captured more precisely using piecewise analyses. Piecewise analyses allow for distinct phases of change to be represented by separate models. In other words, piecewise models consider change as occurring in two phases (Hesser, 2015). Therefore, Piece 1 was conceptualized as change from baseline to writing session 3 (intervention phase), and Piece 2 was conceptualized as change from visits 3 to 5 (follow-up phase). Given the novelty of the current study, and consistent with prior research, it was deemed important to also analyze change in outcomes using piecewise models, to more precisely capture immediate and short-term intervention effects, and help identify potential mechanisms of change of each intervention (Gallop \& Tasca, 2009).

Planned contrasts, with Bonferroni corrections, were conducted in both sets of HLM analyses (total study duration and piecewise), irrespective of significant omnibus tests. This approach was deemed necessary for a number of reasons. First, given that omnibus tests do not 
directly address research questions, lack of planned contrasts may lead researchers to miss important results (Hancock \& Klockars, 1996). Second, whereas the omnibus test reveals differences in general, it does not provide information about specific within-condition changes (Furr, 2008). Therefore, planned contrasts were employed to examine within-condition changes (i.e., significance of slopes within each condition). This approach is consistent with strategies adopted to test within condition changes, and was considered important for elucidating whether each condition exhibited significant patterns of change (Furr, 2008).

\section{Manipulation Check}

Two separate manipulation checks were put in place to assess the degree to which participants followed writing instructions. First, the principal investigator read the contents of each participant's set of scripts alongside an evaluator blind to the hypotheses and assigned conditions. Scripts were categorized according to which of the three writing instructions they adhered to (i.e., written exposure with rescripting, worst-case scenario exposure, neutral control). Scripts for which content deviated from the writing instructions (e.g., writing about a different worst-case scenario each day; lack of a concrete worst-case scenario; writing a vague description of how to move forward from the worst-case scenario) were coded as "not adhering" to instructions. An interrater reliability analysis using the Kappa statistic (Cohen’s $\kappa)$ was performed to determine consistency among raters. The interrater reliability for the raters was found to be $\kappa=.922, p<.01$. Four participants were excluded from all analyses on the grounds that they did not following writing instructions (i.e., wrote about a different worst-case scenario each day; wrote a vague description of a worst-case scenario or how to move forward from the worst-case scenario).

Second, a software program called Linguistic Inquiry and Word Count (LIWC; 
Pennebaker, Booth \& Francis, 2007) was used to analyze the content of scripts by calculating the percentage of words in selected categories (i.e., first person, present tense, emotional and sensory words). ANOVAs were conducted to examine whether participants in the three conditions differed on the following variables: number of words written, proportion of first-person words, proportion of present tense words, proportion of emotion words and proportion of sensory words.

The cumulative number of words across writing sessions was as follows: $\operatorname{RWE}(M=$ 1634 words, $S D=503)$, WE $(M=1651$ words, $S D=448)$, NC $(M=1644$ words, $S D=564)$. Conditions were not significantly different in terms of number of generated words, $p=.99$.

Regarding adherence to instructions of writing in first-person, a two (Pronoun: personal pronouns, impersonal pronoun) by three (Condition: RWE, WE, NC) ANOVA showed a significant main effect of pronoun, $F(1,63)=670.23, p<.01$, with a significantly greater proportion of personal to impersonal pronouns $(M=40.29, S D=0.76 ; M=15.26, S D=0.48)$. Participants within each condition used a significantly greater proportion of personal to impersonal pronouns, $p<.01$. In addition, participants in both exposure conditions (RWE, WE) used a significantly greater proportion of personal pronouns $(M=40.48, S D=5.74 ; M=45.19$, $S D=7.25)$ than did those in the NC $(M=35.20, S D=5.43), p<.01$. There were no differences between the exposure conditions and no significant Pronoun x Condition interaction.

Regarding adherence to instructions of writing in present tense, a three (Tense: Present, Future, Past) by three (Condition: RWE, WE, NC) ANOVA showed a significant main effect of Tense, $F(2,63)=1481.22, p<.01$, with a significantly greater proportion of present tense words $(M=43.06, S D=8.43)$ than future tense or past tense words $(M=5.82, S D=2.55 ; M=6.20$, $S D=3.08$ ). Participants within each condition used a significantly greater proportion of present tense to future or past tense words, $p<.01$. Participants in both exposure conditions (RWE, WE) 
used a significantly greater proportion of present tense words $(M=43.99, S D=1.39 ; M=48.53$, $S D=1.45)$ than did those in the NC $(M=35.91, S D=1.52), p<.01$. Between the exposure conditions, participants in the WE condition used a significantly greater proportion of Present Tense words $(M=48.53, S D=1.45)$ than did participants in the RWE condition $(M=43.99, S D$ $=1.39)$.

Regarding proportion of emotion words, a two (Emotion: Negative, Positive) by three (Condition: RWE, WE, NC) ANOVA showed a significant main effect of Emotion, $F(1,63)=14.17, p<.01$, with a significantly greater proportion of negative emotion words $(M=$ $7.79, S D=4.75)$ than positive emotion words $(M=5.95, S D=2.82)$. Participants in both exposure conditions (RWE, WE) included more negative emotion words in their narratives (i.e., sad, anxious, angry) $(M=10.12, S D=3.51 ; M=10.73, S D=2.40)$ than did those in the NC $(M$ $=1.79, S D=1.13), p<.01$. There were no differences between the exposure conditions. Regarding proportion of positive emotion words, participants in the RWE included more positive emotion words in their narratives (i.e., happy, excited) $(M=7.76, S D=2.82), p<.01$ than did those in the WE condition or NC condition $(M=5.37, S D=2.29 ; M=4.40, S D=2.16)$.

Regarding reference to sensory experiences (e.g., seeing, tasting), one-way ANOVAs showed a significant difference between conditions in the proportion of sensory to total words, $F$ $(2,65)=29.73, p<.01$. Participants in the RWE condition and participants in the WE condition included more references to sensory experiences in their narratives $(M=27.53, S D=6.19 ; M=$ 26.73, $S D=4.42)$ than did those in the NC $(M=16.53, S D=4.46), p<.01$. There were no differences between the exposure conditions.

Overall, it appeared that participants in the exposure conditions followed instructions to include the following elements in their writing: writing in first-person and present tense, 
referencing emotion and sensory experiences. Participants in the neutral condition were not instructed to include these elements and had significantly smaller proportion of words representative of these elements.

\section{Between-Condition Differences at Baseline}

Table 2 displays means and standard deviations for SAM ratings at baseline and each writing session separated by condition. One-way ANOVAS were used to test for baseline differences between conditions. There were significant between-condition differences neither on SAM arousal nor on SAM affect ratings at baseline. Table 3 displays means and standard deviations for GAD symptoms and processes at baseline, Visit 3, 1-week, and 1-month follow-up by condition. One-way ANOVAS were used to test for baseline differences on outcome measures between conditions. There were no significant differences between conditions at baseline.

\section{Objective 1: Worry, GAD Symptoms, Anxiety and Depressive Symptoms}

Worry. Results of HLM analyses for the PSWQ are reported in Tables 4, 5 and 6.

$\boldsymbol{R W E}$ vs. NC. In the RWE vs. NC comparison (Table 4), there was a main effect of time, as evidenced by a significant decline in PSWQ scores over the total study duration, but no main effect of condition, or interaction of Time $\mathrm{x}$ Condition. When examining change within each condition, only participants in the RWE condition showed significant reductions in PSWQ scores. The results of the piecewise analyses indicated a main effect of time during the intervention phase (i.e., visits 1-3), but no significant main effects during the follow-up period. Contrasts revealed that participants in both conditions reported significant reductions in PSWQ scores during the intervention period, although the magnitude of change was greater for participants in the RWE condition, $b=-3.78, \mathrm{SE}=1.50$, than in the NC condition, $b=-3.55, \mathrm{SE}=$ 
1.65. Overall, these results indicate that participants in both conditions report significant improvement in worry during the intervention period, but scores do not continue to improve during follow-up.

WE vs. $\boldsymbol{N C}$. In the WE vs. NC comparison (Table 5), there was a significant decline in PSWQ scores over the total study duration, but no main effect of condition, or interaction of Time $x$ Condition. When examining change within each condition, only participants in the WE condition showed significant reductions in PSWQ scores. The results of the piecewise analyses indicated a main effect of time during both the intervention phase and follow-up period.

Contrasts revealed that participants in both conditions reported significant reductions in PSWQ scores during the intervention period, although the magnitude of change was greater for participants in the WE condition, $b=-3.60, \mathrm{SE}=1.52$, than in the NC condition, $b=-2.81, \mathrm{SE}=$ 1.41. However, only participants in the WE condition showed significant reductions in PSWQ scores during the follow-up period. Overall, these results indicate that participants in both conditions report improvement in worrying during the intervention period, but only participants in the WE condition continue to improve during follow-up.

$\boldsymbol{R} \boldsymbol{W E} \boldsymbol{v s}$. WE. In the RWE vs. WE comparison (Table 6), there was a significant decline in PSWQ scores over the total study duration, but no main effect of condition, or interaction of Time $\mathrm{x}$ Condition. When examining change within each condition, participants in both conditions showed significant reductions in PSWQ scores, although the magnitude of change was greater for participants in the WE condition $b=-2.32, \mathrm{SE}=0.87$, than in the RWE condition, $b=-1.60, \mathrm{SE}=0.66$. The results of the piecewise analyses indicated a main effect of time during the intervention phase, but not the follow-up period. Contrasts revealed that participants in both conditions reported significant reductions in PSWQ scores during the intervention period, 
although the magnitude of change was greater for participants in the RWE condition, $b=-3.78$, $\mathrm{SE}=1.25$, than in the WE condition, $b=-3.60, \mathrm{SE}=1.52$. During the follow-up period, only participants in the WE condition continued to show significant decline in PSWQ scores. Overall, these results indicate that participants in both conditions report significant improvement in worry during the intervention phase, but only participants in the WE condition continue to show significant improvements during follow-up.

GAD symptoms. Results of HLM analyses for the GAD-Q-IV are reported in Tables 7, 8 , and 9 .

$\boldsymbol{R W E} \boldsymbol{v s .} \boldsymbol{N C}$. In the RWE vs. NC comparison (Table 7), there were no main effects of time, condition, or interaction of Time x Condition. When examining change within each condition, contrasts revealed that the only participants in the RWE condition showed significant reductions in GAD-Q-IV scores over the total study duration. The piecewise analyses and contrasts resulted in nonsignificant findings.

$\boldsymbol{W E}$ vs. $\boldsymbol{N C}$. In the WE vs. NC comparison (Table 8), there were no main effects of time, condition, or interaction of Time x Condition. When examining change within each condition, only participants in the WE condition showed significant reductions in GAD-Q-IV scores over the total study duration. The piecewise analyses and contrasts resulted in nonsignificant findings.

$\boldsymbol{R W E} \boldsymbol{v s} . \boldsymbol{W E}$. In the RWE vs. WE comparison (Table 9), there was a main effect of time over the total study duration, but no main effects of condition or interaction of Time $\mathrm{x}$ Condition. Contrasts revealed that participants in the RWE and WE conditions showed significant reductions in GAD-Q-IV scores over the total study duration. The piecewise analyses and contrasts resulted in nonsignificant findings.

Trait Anxiety and depressive symptoms. Results of HLM analyses for the STICSA-T 
and CES-D-R are reported in Tables 10 to 15.

\section{Trait Anxiety.}

$\boldsymbol{R W E} \boldsymbol{v s}$. NC. In the RWE vs. NC comparison (Table 10), there were no main effects of time, condition, interaction of Time $\mathrm{x}$ Condition or contrasts over the total study duration. The piecewise analyses and contrasts resulted in nonsignificant findings.

$\boldsymbol{W E} \boldsymbol{v s} . \boldsymbol{N C}$. In the WE vs. NC comparison (Table 11), there were no main effects of time, condition, interaction of Time $\mathrm{x}$ Condition or contrasts over the total study duration. The results of the piecewise analyses indicated a main effect of time during the intervention phase only. Contrasts revealed that only participants in the WE condition showed significant reductions in STICSA-T scores during the intervention period. Overall, these results indicate improvement in participants' STICSA-T scores for the WE condition during the intervention phase, but scores do not continue to improve during follow-up.

$\boldsymbol{R} \boldsymbol{W E} \boldsymbol{v} \boldsymbol{s} . \boldsymbol{W E}$. In the RWE vs. WE comparison (Table 12), there were no main effects of time, condition, interaction of Time x Condition or contrasts over the total study duration. The piecewise analyses indicated no main effects of time, condition, or interaction of Time $\mathrm{x}$ Condition. Contrasts revealed that only participants in the WE condition showed significant reductions in STICSA-T scores during the intervention period.

\section{Depressive Symptoms.}

$\boldsymbol{R W E} \boldsymbol{v s} . \boldsymbol{N C}$. In the RWE vs. NC comparison (Table 13), there were no main effects of time, condition, interaction of Time x Condition or contrasts over the total study duration. The piecewise analyses and contrasts resulted in nonsignificant findings.

$\boldsymbol{W E}$ vs. $\boldsymbol{N C}$. In the WE vs. NC comparison (Table 14), there were no main effects of time, condition, interaction of Time $\mathrm{x}$ Condition or contrasts over the total study duration. The 
piecewise analyses and contrasts resulted in nonsignificant findings.

$\boldsymbol{R W E} \boldsymbol{v s}$. WE. In the RWE vs. WE comparison (Table 15), there were no main effects of time, condition, interaction of Time $\mathrm{x}$ Condition or contrasts over the total study duration. The piecewise analyses and contrasts resulted in nonsignificant findings.

\section{Objective 2: GAD Cognitive Processes}

Results of HLM analyses for the cognitive processes are reported in Tables 16 to 30.

\section{Intolerance of uncertainty.}

$\boldsymbol{R W E} \boldsymbol{v s .} \boldsymbol{N C}$. In the RWE vs. NC comparison (Table 16), there were no main effects of time, condition, interaction of Time x Condition or contrasts over the total study duration. The piecewise analyses and contrasts resulted in nonsignificant findings.

$\boldsymbol{W E}$ vs. $\boldsymbol{N C}$. In the WE vs. NC comparison (Table 17), there were no main effects of time, condition, interaction of Time $\mathrm{x}$ Condition or contrasts over the total study duration. The piecewise analyses and contrasts resulted in nonsignificant findings.

$\boldsymbol{R W E} \boldsymbol{v s}$. WE. In the RWE vs. WE comparison (Table 18), there were no main effects of time, condition, interaction of Time $\mathrm{x}$ Condition or contrasts over the total study duration. The piecewise analyses and contrasts resulted in nonsignificant findings.

\section{Cognitive avoidance.}

$\boldsymbol{R W E} \boldsymbol{v s} . \boldsymbol{N C}$. In the RWE vs. NC comparison (Table 19), there were no main effects of time, condition, interaction of Time $\mathrm{x}$ Condition or contrasts over the total study duration. The piecewise analyses and contrasts resulted in nonsignificant findings.

$\boldsymbol{W E}$ vs. $\boldsymbol{N C}$. In the WE vs. NC comparison (Table 20), there were no main effects of time, condition, interaction of Time $\mathrm{x}$ Condition or contrasts over the total study duration. The results of the piecewise analyses indicated a main effect of time during the follow-up period only. 
Contrasts revealed that only participants in the WE condition showed significant reductions in CAQ scores during the follow-up period. Overall, these results indicate that participants in the WE condition report significant improvements in CAQ scores during the follow-up period, but not during the intervention period.

$\boldsymbol{R} \boldsymbol{W E} \boldsymbol{v} \boldsymbol{s} . \boldsymbol{W E}$. In the RWE vs. WE comparison (Table 21), there were no main effects of time, condition, interaction of Time x Condition or contrasts over the total study duration. The results of the piecewise analyses indicated no main effects of Time, Condition, or interaction of Time x Condition. Contrasts revealed that only participants in the WE condition showed significant reductions in CAQ scores during the follow-up period.

Negative problem orientation.

$\boldsymbol{R W E} \boldsymbol{v s} . \boldsymbol{N C}$. In the RWE vs. NC comparison (Table 22), there were no main effects of time, condition, interaction of Time x Condition or contrasts over the total study duration. Results of the piecewise analyses indicated a main effect of time during the intervention phase, but no significant main effects during the follow-up period. Contrasts revealed that only participants in the RWE condition showed significant reductions in NPOQ scores during the intervention phase. Overall, these results indicate improvement for participants in the RWE condition during the intervention phase, but no further improvement during follow-up. In contrast, participants in the NC condition show no change on ratings of negative problem orientation.

$\boldsymbol{W E} \boldsymbol{v} \boldsymbol{s} . \boldsymbol{N C}$. In the WE vs. NC comparison (Table 23), there were no main effects of time, condition, interaction of Time $\mathrm{x}$ Condition or contrasts over the total study duration. The piecewise analyses and contrasts resulted in nonsignificant findings.

$\boldsymbol{R} \boldsymbol{W} \boldsymbol{E} \boldsymbol{v}$. $\boldsymbol{W E}$. In the RWE vs. WE comparison (Table 24), there were no main effects of 
time, condition, interaction of Time x Condition or contrasts over the total study duration. Results of the piecewise analyses indicated a main effect of time during the intervention phase, but no significant main effects during the follow-up period. Contrasts revealed that only participants in the RWE condition showed significant reductions in NPOQ scores during the intervention phase. Overall, these results indicate that participants in the RWE condition improved on the NPOQ during the intervention phase, but they did not improve further during follow-up. In contrast, participants in the WE condition showed no changes in ratings on the NPOQ.

\section{Fear of Emotion.}

$\boldsymbol{R W E} \boldsymbol{v s} . \boldsymbol{N C}$. In the RWE vs. NC comparison (Table 25), there was a main effect of time over the total study duration, but no main effect of condition, or interaction of Time $\mathrm{x}$ Condition. Contrasts revealed that only participants in the RWE condition showed significant reductions in ACS scores. Results of the piecewise analyses indicated a main effect of time during the intervention phase. Contrasts revealed that only participants in the RWE condition showed significant reductions in ACS scores during the intervention period.

$\boldsymbol{W E}$ vs. $\boldsymbol{N C}$. In the WE vs. NC comparison (Table 26), there was a main effect of time and an interaction of Time $\mathrm{x}$ Condition over the total study duration, meaning that the condition had a significant influence on the strength of the relationship between time and ACS scores. Contrasts revealed that only participants in the WE condition showed significant reductions in ACS scores. The rate of change between conditions was significantly different, with participants in the WE condition showing significantly greater changes in ACS scores $(b=-3.63, \mathrm{SE}=1.58)$ than participants in the NC condition $(b=0.95, \mathrm{SE}=1.41)$. Results of the piecewise analyses indicated a main effect of time during the intervention phase. Contrasts revealed that only 
participants in the WE condition showed significant reductions in ACS scores during the intervention period. Overall, these results indicate that participants in the WE condition showed significantly greater reductions in fear of emotion and attempts to control emotional experiences compared to participants in the NC condition, who showed no improvement.

$\boldsymbol{R} \boldsymbol{W E} \boldsymbol{v s}$. WE. In the RWE vs. WE comparison (Table 27), there was a main effect of time over the total study duration, but no main effect of condition, or interaction of Time $\mathrm{x}$ Condition. Contrasts revealed that participants in both exposure conditions showed significant reductions on ACS scores of the total study duration, but the magnitude of change was greater for participants in the WE condition $(b=-3.61, \mathrm{SE}=1.54)$ than the RWE condition $(b=-2.60$, $\mathrm{SE}=7.73$ ). Results of the piecewise analyses indicated a main effect of time during the intervention phase. Contrasts revealed that participants in both conditions showed significant reductions in ACS scores during the intervention period, but the magnitude of change was greater for participants in the WE condition $(b=-7.27, \mathrm{SE}=2.95)$ than the RWE condition $(b=$ $-6.52, \mathrm{SE}=2.98)$.

\section{Fear of anxiety.}

$\boldsymbol{R} \boldsymbol{W} \boldsymbol{E} \boldsymbol{v} \boldsymbol{s} . \boldsymbol{N C}$. In the RWE vs. NC comparison (Table 28), there were no main effects of time, condition, interaction of Time x Condition or contrasts over the total study duration. The piecewise analyses and contrasts resulted in nonsignificant findings.

$\boldsymbol{W E} \boldsymbol{v} \boldsymbol{s} . \mathbf{N C}$. In the WE vs. NC comparison (Table 29), there were no main effects time, condition, interaction of Time x Condition or contrasts over the total study duration. The piecewise analyses and contrasts resulted in nonsignificant findings.

$\boldsymbol{R} \boldsymbol{W} \boldsymbol{E} \boldsymbol{v}$. $\boldsymbol{W E}$. In the RWE vs. WE comparison (Table 30), there were no main effects of time, condition, interaction of Time x Condition or contrasts over the total study duration. The 
piecewise analyses and contrasts resulted in nonsignificant findings.

Exploratory analyses: Fear of other emotions. Although there were no a priori hypotheses regarding change on the other subscales of the Affective Control Scale, HLM analyses were performed to determine whether fear of any other emotions improved over the course of the study. Results of the HLM analyses are reported in Tables 31 to 39.

\section{Fear of anger.}

$\boldsymbol{R W E} \boldsymbol{v s} . \boldsymbol{N C}$. In the RWE vs. WE comparison (Table 31), there was a main effect of time over the total study duration, but no main effects of condition, or interaction of Time $\mathrm{x}$ Condition. When examining change within each condition, only participants in the RWE condition showed significant reductions in fear of anger scores. Results of the piecewise analyses indicated a main effect of time during the intervention phase. Contrasts revealed that only participants in the RWE condition showed significant reductions in fear of anger during the intervention period. Overall, these results indicate significant reductions in fear of anger for participants in the RWE condition, but not the NC condition.

$\boldsymbol{W E}$ vs. $\boldsymbol{N C}$. In the WE vs. NC comparison (Table 32), there were no main effects of time, condition, interaction of Time x Condition or contrasts over the total study duration. The piecewise analyses and contrasts resulted in nonsignificant findings.

$\boldsymbol{R W E} \boldsymbol{v s}$. WE. In the RWE vs. WE comparison (Table 33), there was a main effect of time over the total study duration, but no main effects of condition, or interaction of Time $\mathrm{x}$ Condition. When examining change within each condition, only participants in the RWE condition showed significant reductions in fear of anger scores. Results of the piecewise analyses indicated a main effect of time during the intervention phase. Contrasts revealed that only participants in the RWE condition showed significant reductions in fear of anger during the 
intervention period. Overall, these results indicate significant reductions in fear of anger for participants in the RWE condition, but not the WE condition.

\section{Fear of depressed mood.}

$\boldsymbol{R W E} \boldsymbol{v}$ s. $\boldsymbol{N C}$. In the RWE vs. WE comparison (Table 34), there were no main effects of time, condition, interaction of Time x Condition or contrasts over the total study duration. The piecewise analyses and contrasts resulted in nonsignificant findings.

$\boldsymbol{W E} \boldsymbol{v} \boldsymbol{s} . \boldsymbol{N C}$. In the WE vs. NC comparison (Table 35), there were no main effects of time, condition, interaction of Time x Condition or contrasts over the total study duration. The piecewise analyses and contrasts resulted in nonsignificant findings.

$\boldsymbol{R} \boldsymbol{W} \boldsymbol{E} \boldsymbol{v}$. $\boldsymbol{W E}$. In the RWE vs. WE comparison (Table 36), there were no main effects of time, condition, interaction of Time x Condition or contrasts over the total study duration. The piecewise analyses and contrasts resulted in nonsignificant findings.

\section{Fear of positive emotion.}

$\boldsymbol{R W E} \boldsymbol{v s} . \boldsymbol{N C}$. In the RWE vs. NC comparison (Table 37), there was a main effect of time over the total study duration, but no effect of condition or interaction of Time x Condition. Contrasts revealed that only participants in the RWE condition showed significant reductions in fear of positive emotion. The results of the piecewise analyses indicated a main effect of time during the intervention phase. Contrasts revealed that only participants in the RWE condition showed significant reductions in fear of positive emotion during the intervention phase. Overall, these results indicate significant reductions in fear of positive emotion for participants in the RWE condition, but no change for those in the NC condition.

$\boldsymbol{W E} \boldsymbol{v s} . \boldsymbol{N C}$. In the WE vs. NC comparison (Table 38), there were no main effects of time condition, interaction of Time x Condition or contrasts over the total study duration. The results 
of the piecewise analyses indicated a main effect of time during the intervention phase. Contrasts revealed that only participants in the WE condition showed significant reductions in fear of positive emotion during the intervention phase.

$\boldsymbol{R} \boldsymbol{W E} \boldsymbol{v} \boldsymbol{s} . \boldsymbol{W E}$. In the RWE vs. WE comparison (Table 39), there were no main effects of time, condition, interaction of Time $\mathrm{x}$ Condition or contrasts over the total study duration. The results of the piecewise analyses indicated a main effect of time during the intervention phase. Contrasts revealed that participants in both conditions reported significant reductions in fear of positive emotion during the intervention phase, although the magnitude of change was greater for participants in the RWE condition $b=-3.70, \mathrm{SE}=1.48$, than in the WE condition, $b=-3.17, \mathrm{SE}=$ 1.42.

Perceived probability. Results of the HLM analyses for perceived probability are reported in Tables 39 to 41 .

$\boldsymbol{R} \boldsymbol{W E} \boldsymbol{v s}$. $\boldsymbol{N C}$. In the RWE vs. WE comparison (Table 40), there were no main effects of time, condition, interaction of Time $\mathrm{x}$ Condition or contrasts over the total study duration. The piecewise analyses and contrasts resulted in nonsignificant findings.

$\boldsymbol{W E}$ vs. $\boldsymbol{N C}$. In the WE vs. NC comparison (Table 41), there were no main effects of time or condition, but there was a significant interaction of Time $\mathrm{x}$ Condition over the total study duration, indicating that the rate of change between conditions was significantly different. Contrasts revealed that only participants in the WE condition showed significant reductions in perceived probability scores (i.e., perceived probability of the worst fear coming true). The rate of change between conditions was significantly different, with participants in the WE condition showing significantly greater reductions in perceived probability $(b=-0.14, \mathrm{SE}=0.09)$, compared to participants in the NC condition, $(b=0.12, \mathrm{SE}=0.008)$. The piecewise analyses and contrasts 
resulted in nonsignificant findings. Overall, results indicate that participants in the WE condition showed significantly greater reductions in perceived probability of the worst fear coming true, compared to participants in the NC condition, who showed no improvement.

$\boldsymbol{R W E} \boldsymbol{v s}$. WE. In the RWE vs. WE comparison (Table 42), there were no main effects of time, condition, interaction of Time $\mathrm{x}$ Condition or contrasts over the total study duration. The piecewise analyses and contrasts resulted in nonsignificant findings.

Perceived cost. Results of the HLM analyses for perceived cost are reported in Tables 42 to 44 .

$\boldsymbol{R W E} \boldsymbol{v s}$. NC. In the RWE vs. NC comparison (Table 43), there was a main effect of time over the total study duration, but no main effect of condition or Time $\mathrm{x}$ Condition interaction. When examining change within each condition, only participants in the RWE condition showed significant reductions in perceived cost scores. The results of the piecewise analyses indicated a main effect of time and a significant Time $\mathrm{x}$ Condition interaction during the intervention phase. Contrasts revealed that only participants in the RWE condition showed significant reductions in perceived cost scores during the intervention phase. The rate of change between conditions was significantly different, with participants in the RWE condition showing significantly greater reductions in perceived cost scores $(b=-0.47, \mathrm{SE}=0.23)$ than participants in the NC condition $(b=0.19, \mathrm{SE}=0.25)$ during the intervention phase. Overall, results indicate that participants in the RWE condition showed improvements in perceived cost scores (i.e., they perceived their worst case scenario as less "bad"), and that reductions in these scores were significantly greater for participants in the RWE condition versus the NC condition during the intervention phase of the study.

$\boldsymbol{W E} \boldsymbol{v s} . \boldsymbol{N C}$. In the WE vs. NC comparison (Table 44), there were no main effects of time, 
condition, interaction of Time $\mathrm{x}$ Condition or contrasts over the total study duration. The piecewise analyses and contrasts resulted in nonsignificant findings.

$\boldsymbol{R W E} \boldsymbol{v s}$. WE. In the RWE vs. WE comparison (Table 45), there was a main effect of time over the total study duration, but no main effect of condition, or interaction of Time $\mathrm{x}$ Condition. When examining change within each condition, only participants in the RWE condition showed significant reductions in perceived cost scores. The results of the piecewise analyses indicated a main effect of time during the intervention phase, but no significant main effects during the follow-up period. Contrasts revealed that only participants in the RWE condition showed significant reductions in perceived cost scores during the intervention period. Overall, these results indicate that at the end of the intervention period, individuals assigned to the RWE condition appraised their worst case scenario as less "bad" relative to pre-intervention; however, there were no further changes at follow up. In contrast, those assigned to the WE condition reported no change in the perception of the "badness" of their worst-case scenario following the intervention or at follow-up.

Perceived coping. Results of the HLM analyses for perceived coping are reported in Tables 46 to 48 .

$\boldsymbol{R W E} \boldsymbol{v s} . \boldsymbol{N C}$. In the RWE vs. NC comparison (Table 46), there was no main effect of time, condition, Time $\mathrm{x}$ Condition interaction or contrasts over the total study duration. The results of the piecewise analyses indicated a main effect of time, and a significant Time $\mathrm{x}$ Condition interaction during the intervention phase. Contrasts revealed that only participants in the RWE condition showed significant increases in perceived coping scores during the intervention phase. The rate of change between conditions was significantly different, with participants in the RWE condition showing significantly greater increases in perceived coping 
scores $(b=1.08, \mathrm{SE}=0.34)$ than participants in the $\mathrm{NC}$ condition $(b=-0.25, \mathrm{SE}=0.38)$ during the intervention phase. Overall, results indicate that only participants in the RWE condition report increases in their perceived ability to cope with their worst fear coming true during the intervention period, and that improvements in these scores were significantly greater for participants in the RWE condition versus the NC condition during the intervention phase of the study.

$\boldsymbol{W E}$ vs. $\boldsymbol{N C}$. In the WE vs. NC comparison (Table 47), there were no main effects of time, condition, interaction of Time $\mathrm{x}$ Condition or contrasts over the total study duration. The piecewise analyses and contrasts resulted in nonsignificant findings.

$\boldsymbol{R} \boldsymbol{W} \boldsymbol{E} \boldsymbol{v s} . \boldsymbol{W E}$. In the RWE vs. WE comparison (Table 48), there were no main effects of time, condition, interaction of Time $\mathrm{x}$ Condition or contrasts over the total study duration. The results of the piecewise analyses indicated a main effect of time during both the intervention phase and follow-up period. Contrasts revealed that only participants in the RWE condition showed significant increases in perceived coping scores during the intervention phase, whereas participants in the WE condition showed significant decreases in perceived coping scores during the follow-up period. Overall, results indicate that participants in the RWE condition report improvements in their perceived ability to cope with their worst fear coming true during the intervention period. In contrast, participants in the WE condition report significant decreases in their perceived coping abilities during the follow-up phase.

Objective 3: Intolerance of Uncertainty and Negative Problem Orientation as Mediators of Change in Worry 
Intolerance of uncertainty. Tests of mediation were not conducted with IU as a mediator in the RWE and WE conditions, as there were no changes in IUS scores from baseline to follow-up.

Negative problem orientation. Although participants in the RWE condition displayed significant reductions in NPO scores during the intervention period, there were no significant reductions during the follow-up period. In studies with two distinct time periods (e.g., intervention, follow-up), as is the case for the current study, the growth of the mediator and the outcome process is modeled using a two-stage parallel process model, allowing mediated effects to be investigated at different periods (Cheon, MacKinnon \& Khoo, 2003). However, the intervention period consisted of only pre and post assessments (i.e., at baseline and at the end of the third writing session), which is considered inadequate in estimating change over time (Kraemer, Wilson, Fairburn \& Agras, 2002; MacKinnon 2008; Maric, Wiers \& Prins, 2012; Rogosa, 1988). Thus, tests of mediation could not be conducted. 


\section{Discussion}

\section{Purpose of the Study}

The first objective of the present study was to determine whether written exposure with rescripting (RWE) is more efficacious in reducing worry and GAD symptoms than worst-case scenario exposure (WE) or neutral writing (NC). The second objective was to determine the degree to which GAD-related cognitive processes improve following each of the exposure interventions. Lastly, this study sought to examine how the written exposure interventions result in reductions in worry.

\section{Summary of Main Findings}

Results did not support the hypotheses that (1) participants in the exposure conditions would show significantly greater decreases in worry, GAD symptoms, and depressive symptoms compared to participants in the neutral writing condition and (2), that improvements would be most pronounced for participants in the written exposure with rescripting condition. Multilevel models showed that worry significantly decreased in all three conditions during the intervention period, to a similar degree. During the follow-up period, only participants in the worst-case scenario exposure condition continued to show significant reductions in worry. Regarding changes in GAD symptoms, participants in both exposure conditions showed significant decreases on GADQ-IV scores over the total study duration, whereas individuals who engaged in neutral writing did not. There were no significant within condition changes on depressive symptoms in any of the conditions.

Regarding GAD-related cognitive processes, results partially supported the hypotheses that (1) participants in the exposure conditions would show significantly greater improvements in these processes than participants in the neutral control condition and (2), that improvements 
would be most pronounced for participants in the written exposure with rescripting condition. With regard to intolerance of uncertainty, there were no significant changes or between group differences. There were, however, significant improvements on measures of cognitive avoidance, fear of emotion, negative problem orientation, and perceived probability, cost, and coping. Contrary to hypotheses, only participants in the worst-case scenario exposure condition showed significant reductions in cognitive avoidance, and during the follow-up period only. In addition, only participants in the written exposure with rescripting condition showed significant reductions in negative problem orientation scores during the intervention period only. Regarding fear of emotion, participants in both exposure conditions showed significant reductions over the total study duration, whereas participants who engaged in neutral writing did not. In addition, the rate of change for fear of emotion was significantly different between conditions; participants in the worst-case scenario exposure condition reported significantly greater reductions in fear of emotion than those in the neutral condition. However, contrary to the hypothesis, there were no changes or differences in fear of anxiety subscale scores in any of the conditions, thus overall change on fear of emotion does not reflect a change in fear of anxiety. Finally, regarding ratings of perceived probability, cost, and coping, results were mixed, in some cases favoring written exposure with rescripting and in others favoring worst-case scenario exposure. Regarding ratings of perceived probability of the worst fear coming true, there were no changes in ratings of participants in the written exposure with rescripting or neutral conditions. However, participants in the worst-case scenario exposure condition reported significantly greater reductions in perceived probability of the worst fear coming true over the total study duration compared to participants in the neutral condition. Regarding ratings of the perceived cost of the worst fear coming true, only participants in the written exposure with rescripting condition perceived their 
worst-case scenario as less costly at the end of the intervention period, with reductions in these scores being significantly greater for participants in the written exposure with rescripting condition compared to the neutral condition. Finally, regarding ratings of perceived ability to cope with the worst fear coming true, only participants in the written exposure with rescripting condition showed significant improvements over the total study duration, with significantly greater improvements compared to the neutral condition during the intervention period.

Lastly, the hypotheses that change in worry in the exposure conditions would be mediated by changes in intolerance of uncertainty and negative problem orientation could not be tested. There were no changes in intolerance of uncertainty in any of the exposure conditions, and changes in negative problem orientation only during the intervention period meant there were insufficient assessment points to establish mediation.

\section{Past Research on Written Exposure}

Although some of the current findings are in line with prior work on written exposure for worry, there are some discrepancies as well. First, consistent with Fracalanza et al. (2014) and Goldman et al. (2007), the results of the current study also showed that individuals who wrote repeatedly about the same worst fear coming true reported large, significant decreases on the PSWQ from baseline to 1-week and 1-month follow-up $(d=.98$ and $d=.91)$. The effect sizes reported by Fracalanza and colleagues (2014) and Goldman and colleagues (2007) were similar, ( $d=.91$ and $d=1.27$, respectively). In the current study, individuals in the written exposure with rescripting condition also reported significant decreases on the PSWQ from baseline to 1-week and 1-month follow-up, although the effect sizes were smaller $(d=.60$ and $d=.69)$. An unexpected finding in the current study was that participants in the neutral condition experienced significant reductions in worry from baseline to 1-week follow-up $(d=.57)$. Neutral writing 
conditions in other written exposure studies have also resulted in unexpected findings. For example, Goldman et al. (2007) unexpectedly found that participants in their control condition experienced significant reductions in GAD somatic symptoms, and speculated that the decrease may have been due to the rationale they provided to participants, that writing leads to improvement in physical health. Goldman et al. (2007) also suggested that perhaps the reductions in GAD symptoms were due to repeated administration of self-report measures; when repeatedly administered over a short time period, self-report measures have been shown to lead to decreases in mean scores on the measure (e.g., Sharpe \& Gilbert, 1998). Like Goldman et al. (2007), Fracalanza et al. (2014) also found that participants in their control condition showed significant reductions in GAD-associated symptoms, and examined the content of their participants' scripts to elucidate this result. They found that participants in the neutral writing condition wrote about pleasurable, goal-directed activities that they would like to engage in if they had free time. Given the link between engaging in pleasurable, goal-directed activities and reduced depressive symptomatology (Dimidjian, Barrera, Martell, Munoz \& Lewinsohn, 2011), and the overlap between GAD and depressive symptoms, they suggested that perhaps participants in the neutral writing condition actually engaged in the activities they wrote about, which in turn may have improved GAD-associated symptoms. The explanations offered by both Goldman et al. (2007) and Fracalanza et al. (2014) may also be applicable to the current study given the similar methodology (i.e., similar writing instructions, repeated administration of the PSWQ over a short time period). For example, participants in the current study may have expected that their worrying would improve, given they were provided the following rationale: "although research has shown that writing leads to positive health and psychological outcomes, the relationship between writing and worry has not been thoroughly investigated." In other 
words, participants may have perceived the neutral condition to be beneficial. In line with this suggestion, prior work on written disclosure has shown that participants rate neutral writing (i.e., writing about time management and future plans) as a credible technique for reducing stress (Lumley \& Provenzano, 2003; Radcliffe, Lumley, Kendall, Stevenson \& Beltran, 2007). Therefore in the current study, participants' expectancies to change may have played a role in the observed effects of the neutral condition. In line with expectancy to change, other nonspecific factors, such as positive contact with the experimenter, or motivation to change may have also contributed to improved outcome for participants in the neutral condition. It is also important to note that these nonspecific factors were shared by all conditions, and therefore may have accounted for improved outcomes for all participants. This last point is consistent with a large body of research which suggests that nonspecific factors are significant mediators of change in treatment (e.g., Blease, Lilienfeld \& Kelley, 2016; Cuijpers, 2016).

To further elucidate why participants in the neutral condition of the current study reported reduced worrying, the content of neutral scripts was examined. Even though participants were instructed to write about what they would do with the day off in a factual, unemotional way, all participants in the neutral condition wrote about pleasurable or goal-directed activities that they would like to engage in if they had the day off. Some examples of goal-directed activities participants wrote about were finishing personal or school projects, studying, organizing, cleaning living spaces or exercising. Research has shown that goal-directed activities have positive psychological consequences because they help people organize, prioritize and manage motives (Shah \& Kruglanski, 2008). More specific to the current study, research has shown that writing about goals may be beneficial because it promotes a sense of accomplishment (King, 2001). Participants also wrote about engaging in pleasurable activities, such as cooking a 
favorite meal, watching favorite television shows, and seeing friends. Many of these topics are inherently positive experiences and research has shown that individuals who write about positive experiences show enhanced psychological well being (Burton \& King, 2004).

In line with this, examination of Self-Assessment Manikin (SAM) ratings for participants in the neutral condition showed that these participants did in fact find the writing task relaxing and moderately pleasurable. On the SAM arousal scale (ranging from 1 "very calm" to 9 "very aroused"), participants in the neutral condition had a mean rating of $3.57(S D=1.26)$. On the SAM affect scale (ranging from 1 "very pleasant" to 9 "very unpleasant"), participants in the neutral condition had a mean rating of $4.40(S D=1.01)$.

Taken together, these findings suggest that the neutral condition may have in fact been more positive than neutral. In a recent study, researchers asked participants with GAD to either (1) practice replacing the usual form of worry with images of possible positive outcomes, (2) with the same positive outcomes represented verbally or (3) a control condition which involved generating any positive images (Eagleson, Hayes, Mathews, Perman \& Hirsch, 2016).

Interestingly, they found that worrying was reduced in all three conditions. That is, any positive ideation reduced the frequency of worry. Therefore, perhaps in the current study, the positive content in the neutral scripts served a similar function.

In summary, writing about the way one would spend one's time on a day off may have influenced scores on the PSWQ for the following reasons: (1) participant may have expected to improve, (2) it may have facilitated goal-setting and attainment, (3) it may have been experienced as a pleasurable activity and (4) it may have encouraged the promotion of positive ideation. Finally, the current findings and those of prior studies (e.g., Fracalanza et al., 2014; Goldman et al., 2007) also raise questions as to whether the neutral writing condition is truly 
neutral in its effects; future studies on written exposure may wish to vary instructions in the neutral condition as to not encourage writing about potentially positive experiences.

The current study also had some key differences compared to prior work on written exposure. Both Fracalanza et al. (2014) and Goldman et al. (2007) found significant improvements in intolerance of uncertainty from baseline to follow-up $(d=0.72$ and $d=0.44$, respectively), whereas in the current study there was improvement neither in the worst-case scenario exposure condition nor in the written exposure with rescripting condition $(d=0.03$ and $d=0.19$, respectively). There are a few potential explanations for this discrepancy. First, the sample in Goldman et al.'s (2007) study was not a clinical sample, and instead was a sample of individuals high in worry (mean PSWQ score $=58.26, S D=7.04$ ). In addition, participants in Goldman et al.'s study received five, 30-minute sessions of written exposure to the worst-case scenario, whereas participants in the current study only received three. Finally, findings from Goldman et al. (2007) must be interpreted with caution, as there were significant baseline differences between conditions on the Intolerance of Uncertainty Scale, with participants in the written exposure condition scoring significantly higher than participants in the neutral control condition. Therefore, it is unclear whether improvement was due to the written exposure intervention, or because participants in the exposure condition had more room for improvement. These initial differences between conditions limit conclusions regarding the efficacy of written exposure on intolerance of uncertainty, as they obscure whether there are any real differences in the outcome. Second, although participants in Fracalanza et al.'s (2014) study and the current study reported symptoms that were consistent with a principal diagnosis of GAD, mean PSWQ and IUS scores in the current sample $(M=69.13, S D=4.98 ; M=89.11, S D=18.97$, respectively) were higher than those in Fracalanza et al.'s (2014) $(M=63.09, S D=8.07 ; M=$ 
$84.03, S D=20.66)$. Research has shown that intolerance of uncertainty and excessive worry are highly correlated (e.g., Buhr \& Dugas, 2006; Dugas, Freeston \& Ladouceur, 1997), and that individuals who do not improve following $\mathrm{CBT}$ for GAD continue to endorse elevated levels of intolerance of uncertainty (e.g., Donegan \& Dugas, 2012; Dugas, Brillon, Savard et al., 2010). This suggests that at higher tendencies to worry excessively and uncontrollably, as was the case for participants in the current study, intolerance of uncertainty may be less likely to change.

\section{Changes in Fear of Emotion}

Results showed that over the total study duration, participants in the worst-case scenario exposure condition reported significantly greater improvements on the Affective Control Scale compared to participants in the neutral control condition. These findings suggest that repeatedly writing about the same feared scenario is associated with decreased fear of emotions, and are in line with several theories of worry which suggest that individuals with GAD experience negative reactions to emotions and prefer to avoid them. For example, according to Borkovec's Avoidance Theory of Worry, worry is a verbal-linguistic maladaptive strategy used to avoid emotional arousal associated with images of the worst-fear (Borkovec et al., 2004). The Avoidance Theory has served as a foundation for subsequent theories of GAD, such as the Emotion Dysregulation Model, which extended it to suggest that worry enables emotional avoidance. This model states that individuals with GAD experience heightened intensity of emotions, experience emotions as threatening, and in turn attempt to avoid or suppress them (Mennin et al., 2005). Heightened intensity and perceived uncontrollability of emotions have been shown to be especially strong predictors of GAD (e.g., Mennin, Holaway, Fresco, Moore \& Heimberg, 2007; Mennin, McLaughlin \& Flanagan, 2009; Stapinski, Abbott \& Rapee, 2010; Turk, Heimberg, Luterek, Mennin \& Fresco, 2005). From the perspective of these theories, 
repeatedly writing about the same worst fear may have led to reduced attempts at avoiding feared emotions via worrying. This possibility was investigated by conducting a posthoc regression analysis to examine whether a change in fear of emotion predicted change in worry in the worstcase scenario exposure condition. As noted, both worry and fear of emotions were significantly reduced over the course of the study for participants who engaged in worst-case scenario exposure. However, there was a nonsignificant indirect effect of worst-case scenario exposure on worry through fear of emotion, $b=0.10$, BCa CI $[-0.05,0.58]$. Therefore, change in fear of emotion did not significantly predict change in worry in the worst-case scenario exposure condition. Despite this, the findings offer novel insights. Prior studies have not examined the effects of the written exposure intervention on fear of emotions, and these new findings suggest that writing repeatedly about a worst-fear coming true is associated with decreased fear of emotional states.

Results of the current study also showed that participants in the written exposure with rescripting condition reported reductions in fear of emotions over the total study duration, albeit to a lesser degree than did participants in the worst-case scenario exposure condition; possible explanations are proposed to explain this result. First, the smaller degree of change on the Affective Control Scale for participants in the written exposure with rescripting condition could be understood from the context of Foa and Kozak's (1986) Emotional Processing Theory. This theory explains that for emotional processing of a feared situation to occur, it must involve activation of the feared stimulus, and corrective information about maladaptive beliefs about the feared stimulus. If emotions are the feared stimuli, then accessing, experiencing, and expressing such emotions is fundamental for successful emotional processing (Foa \& Kozak, 1986). Posthoc analyses of SAM data (arousal and affect) in the current study were conducted to examine 
whether participants in the written exposure with rescripting condition may have received a smaller "dose" of exposure to negative emotions (i.e., anxiety, negative affect) than participants in the worst-case scenario exposure condition. That is, if participants in the written exposure with rescripting condition had significantly lower ratings of anxiety and negative affect than participants in the worst-case scenario exposure condition, it may indicate that they had fewer opportunities to confront these emotions and interpret them in a less threatening way. Results showed that participants in the written exposure with rescripting condition reported significant reductions in anxious arousal from the first to the second writing session, $F(1,63)=21.44 p$ $<.01$, whereas participants in the worst-case scenario exposure condition did not $F(1,63)=0.77$, $p=.38$. Moreover, participants in the written exposure with rescripting condition reported significant reductions in negative affect across sessions, $F(1,62)=11.77, p<.01$, whereas participants in the worst-case scenario exposure condition did not $F(1,62)=1.21, p=.30$. These findings, coupled with the fact that participants in the written exposure with rescripting condition had instructions in the second and third sessions to write about how to move forward and change their attitude about the worst fear, suggest that perhaps participants in this condition received a smaller "dose" of exposure to negative emotions. In line with this, Fracalanza et al. (2014) suggested that emotional processing was more pronounced for individuals who wrote repeatedly about the same feared scenario on three consecutive days, compared to individuals who wrote about different feared scenarios on each of three days. Taken together, these findings support Foa and Kozak's (1986) notion that for fear reduction to occur, maladaptive beliefs must be disconfirmed through repeated confrontation of the feared stimulus.

In summary, the smaller magnitude of change on the Affective Control Scale for participants in the written exposure with rescripting condition may be because they did not have 
enough time to confront feared emotions, thus emotional processing may have been less pronounced.

Thus far, results suggest that the written exposure interventions may be able to target fear of emotions. Yet results of the current study also raise questions as to which emotions are most feared. Although research suggests that anxiety is the most prominent emotion that is feared in individuals with GAD (Mennin et al., 2004), there were no changes on the fear of anxiety subscale in the current study. Research has also shown that individuals with GAD report greater fear of depression than do nonpsychiatric controls (Roemer et al., 2005). Given that the Affective Control Scale also assesses fear of depressed mood, fear of anger, and fear of positive emotions in addition to fear of anxiety, a posthoc HLM analysis was conducted to determine whether there were any improvements on the other subscales that could have accounted for the observed decline on the total score on the Affective Control Scale. In the written exposure with rescripting condition, there was a significant reduction in fear of anger scores and fear of positive emotion scores over the course of the study; there was also a significant reduction in fear of positive emotion scores for participants in the worst-case scenario exposure condition during the intervention period, although the magnitude of change was greater in the written exposure with rescripting condition. These results are interesting, as they suggest that there may be a variety of emotions that individuals with GAD are afraid of and trying to suppress, including anger and positive emotion. Prior research has shown that a heightened level of internalized anger is a strong predictor of GAD, over and above other forms of anger (e.g., physical aggression, verbal aggression (Deschenes, Dugas, Fracalanza \& Koerner, 2012). In addition, Foa, Riggs, Massie and Yarczower (1995) have shown that high levels of anger at the start of imaginal exposure predict a negative outcome. Therefore, it seems important to afford individuals the opportunity to 
express this emotion, particularly because it may have been internalized. It may be that the instructions in the written exposure with rescripting condition provided people with the opportunity to express and reevaluate feelings of anger towards the worst fear. In line with these findings, research in the PTSD literature has shown that imaginal exposure enhanced with rescripting leads to improved outcomes for nonfear PTSD emotions such as anger, guilt, and shame, whereas imaginal exposure alone does not (Arntz, Kindt \& Tiesema, 2007).

The findings pertaining to reductions in fear of positive emotion are also in line with research suggesting that individuals with GAD have an elevated fear of consequences of positive emotion (Mennin et al., 2007). Different explanations can account for the reductions in fear of positive emotion in each of the exposure conditions. Although qualitative analysis of scripts was beyond the scope of this study, closer examination of participants' scripts in the worst-case scenario exposure condition revealed that $16 \%$ of individuals engaged in spontaneous reappraisal of their worst fear. That is, some individuals in the worst-case scenario condition arrived at a more balanced view of their feared situation without being guided to do so, and thus may have been exposed to some positive emotions. However, the results suggest that individuals in the written exposure with rescripting condition showed greater reductions in fear of positive emotion. There are a few reasons why this may be the case. First, the manipulation check showed that participants in the written exposure with rescripting condition indeed referenced positive emotions significantly more than participants assigned to the other conditions, suggesting a greater "dose" of exposure to positive emotion. Second, results can be understood from the perspective of the Contrast Avoidance Model of worry, which explains that individuals with GAD fear positive emotion because they do not want to feel vulnerable and experience a sudden negative shift in emotion (Newman \& Llera, 2011). Perhaps explicitly asking individuals 
in the written exposure with rescripting condition to write about how to move forward and change their attitude toward the worst fear allowed for targeting the belief that positive emotion is threatening or unpleasant, and in turn, fear of positive emotion was decreased.

In summary, the current findings have important clinical implications. First, exposure has been focused exclusively on anxiety reduction. However the current results suggest that it may be especially important for exposure to target a range of feared emotions, especially anger and positive emotion, to help individuals learn that these emotions are tolerable and not dangerous. The findings also highlight the value of written exposure enhanced with rescripting in targeting emotions other than anxiety.

\section{Changes in Negative Problem Orientation}

Results of the current study showed that only participants in the written exposure with rescripting condition improved on negative problem orientation, ratings of the perceived cost of the worst fear coming true, and perceived ability to cope with the worst fear. Negative problem orientation has an important role in Dugas' Cognitive Model of GAD (Dugas et al., 1998), and is one of the most significant predictors of GAD severity, relative to other components of the model (Dugas et al., 2007). Specifically, individuals with high levels of worry report lower problem-solving ability, problem-solving confidence and perceived control over problems than individuals with low levels of worry (Davey, 1994; Dugas et al., 1995; Ladouceur, Blais, Freeston \& Dugas, 1998). Dugas and Robichaud (2007) also note that individuals with GAD do not have an inability to find solutions to problems; rather, they have difficulties arriving at a solution because of their negative problem orientation (i.e., they view problems as threats, doubt their coping abilities, and are pessimistic about problems). In line with this, individuals with GAD tend to overestimate the magnitude of their perceived threats (Berenbaum et al., 2007). In 
particular, individuals with GAD perceive the outcomes of feared scenarios as more costly. If individuals believe their concerns are costly, it may be because they believe the outcomes are less manageable (Provencher, Freeston, Dugas \& Ladouceur, 2000). In turn, they also experience decreased confidence in their ability to effectively cope with problems. In line with these findings, it is not surprising that the written exposure with rescripting condition was better able to target maladaptive cognitions about problems and the capacity to deal with problems, while also changing people's perceptions of the "badness" of their feared situation and their perception of the degree to which they could cope in the event of the worst-case scenario. In fact, a tenet of exposure, as it is delivered in many CBT protocols for GAD (e.g., Craske \& Barlow, 2006; Dugas \& Robichaud, 2007), is that after exposure to a feared scenario, individuals are encouraged to spend time thinking of alternative outcomes. That is, individuals are to list other possible explanations or outcomes for the most feared situation (van der Heiden \& ten Broeke, 2009). Therefore, theoretically, in worry exposure the most effective element may be the reevaluation of the most feared expectation, because it enables individuals to realize that even if their worst fear came true, they would be able to cope (van der Heiden \& ten Broeke, 2009).

In summary, written exposure with rescripting (i.e., explicitly instructing individuals to write about how to move forward from and change their attitude about their worst fear) may be especially important for targeting maladaptive cognitions about problems, versus simply having individuals engage in repeated exposure to the same feared scenario.

\section{Understanding Mechanisms of Change}

The variable effects of each of the exposure interventions suggest that the mechanisms may vary as a function of writing instructions. In order to maximize the beneficial effects of the written exposure interventions, it is essential to understand their unique or common potential 
mechanisms of action.

Cognitive avoidance. Exposure purportedly works by decreasing cognitive avoidance. The worst-case scenario exposure condition was the only one in which participants demonstrated significant reductions in cognitive avoidance. Individuals in the worst-case scenario exposure condition showed significant reductions on the Cognitive Avoidance Questionnaire during the follow-up period only, and a trend toward lower cognitive avoidance over the total study duration $(p=.07)$. Therefore, it is possible that worst-case scenario exposure does target cognitive avoidance, and that improvements in worry over time were facilitated by reductions cognitive avoidance, but that the current sample was not large enough to detect an effect. Lack of significant findings may also reflect a problem with the way cognitive avoidance was measured. The Cognitive Avoidance Questionnaire, used in the current study, is a measure of general tendency to engage in strategies to avoid distressing thoughts and mental images and thus may not have been sensitive to detect changes in acute cognitive avoidance. For example, Fracalanza et al. (2014) also included a measure of acute cognitive avoidance, defined as fear and avoidance of an imagined worst-case scenario, and found that participants who wrote repeatedly about the same worst fear coming true experienced significantly less fear and avoidance of imagining that worst fear. Therefore, perhaps participants in the current study did experience reductions in their desire to avoid imagining their specific worst-case scenario, but the CAQ was unable to capture this change. For participants in the written exposure with rescripting condition, The Emotional Processing Theory may help explain the lack of change on the Cognitive Avoidance Questionnaire. As discussed earlier, it may be that participants in the written exposure with rescripting condition were less able to emotionally process their worst fear, because they had less time than participants in the worst-case scenario exposure condition to confront several aspects 
of the feared scenario. In turn, avoidance was maintained.

Cognitive reappraisal. Cognitive reappraisal can be defined as an emotion regulation strategy that entails positive modification or change in the evaluation of a stressor or of the self (for a review, see Gross and Thompson, 2007). Imagery rescripting is a technique that also involves modifications, but to emotion-inducing mental imagery. In imagery rescripting, images are modified during treatment to increase mastery and change associated negative thoughts, feelings and behaviours (Holmes et al., 2007). For example, in the treatment of depression, imagery rescripting is thought to be an experiential technique with a cognitive rationale; it has been suggested that imagery rescripting may lead to reappraisals of negative experiences, in turn reducing their emotional salience (e.g., Moscovitch, Chiupka \& Gavric 2013; Wheatley \& Hackmann, 2011). Individuals with GAD make several problematic appraisals associated with their worst fears. As discussed previously, individuals with GAD tend to make overestimations of the perceived probability and cost of their worst fears, and doubt their ability to cope with or manage their worst fears (Berenbaum et al., 2007; Dugas et al., 1995; Provencher et al., 2000). Thus, it may be that rescripting one's response to the worst fear coming true gave individuals the opportunity to obtain a new perspective on their worst fear. This is evidenced by the fact that only participants in the written exposure with rescripting condition displayed significant reductions in negative problem orientation, perceived costliness of the feared scenario, as well as reported improvement in their coping ability. It has also been suggested that repeated exposure to an aversive stimulus may lead to its reappraisal (Foa \& Rothbaum, 1998). In the current study, repeated exposure to the same worst-case scenario did lead to rating the feared scenario as less probable, however, ratings of the perceived cost of the scenario, ability to cope with the scenario, and negative problem orientation did not change. Thus, written exposure with rescripting may be 
better able to target a broader range of problematic appraisals associated with the worst fear compared to worst-case scenario exposure. In line with these findings, research on the mechanisms of imagery rescripting in the PTSD literature has shown that a modification of maladaptive trauma-related beliefs is responsible for an overall reduction in PTSD and related distress (Long \& Quevillon, 2009; Long et al., 2011). Results of the current study also provide preliminary evidence that one of the mechanisms of change in written exposure with rescripting may be through modification of GAD-related negative cognitions, particularly related to beliefs about costliness, coping ability, and problem orientation.

Concreteness. Lastly, given that participants in both exposure conditions showed reductions in worry, perhaps there exists a common mechanism of change across the interventions. Recall that individuals with GAD tend to worry about perceived threats in an abstract as opposed to concrete way. Abstract thinking in turn attenuates emotional arousal (Borkovec \& Roemer, 1995) and interferes with problem solving (Stöber, 1998). In contrast, concrete processing involves distinct and specific representations of the feared situation, and enables individuals to prepare for or solve expected negative consequences of problems (Stöber \& Borkovec, 2002). Thus, following CBT for GAD, the more concrete the problem elaborations, the greater the reductions in worry (Stöber and Borkovec, 2002). Writing in an abstract (as opposed to concrete) way is also associated with higher negative mood about a recent failure (Watkins, 2004). In contrast, concrete thinking is thought to encourage emotional processing by evoking emotionally charged mental imagery (Paivio \& Marschark, 1991). Concrete processing has also been shown to reduce emotional reactivity in response to a stressor (Watkins et al., 2008). Watkins et al. (2008) showed in a nonclinical sample, that individuals who were trained to imagine concrete details of emotional scenarios (i.e., imagine the details of what is happening) 
had reduced emotional reactivity to a stressor relative to individuals who engaged in abstract processing of the emotional scenario (i.e., think about the causes, meanings and implications). In an extension of this work, Watkins, Baeyens and Read (2009) trained participants with dysphoria to think concretely, and found that concreteness training resulted in significantly greater decreases in depressive symptoms and rumination, and significantly greater increases in concrete thinking than a wait-list or sham control condition.

In the current study, evidence of concrete processing in the exposure conditions can be gleaned from participants' instructions and subsequent emotional reactions during writing. For example, participants in both exposure conditions were instructed to write a detailed description of their worst fear coming true in first-person, present tense, with references to sensory and emotional reactions, which may have helped concretize the feared scenario. Concrete problem definitions are fundamental to the development of effective problem solutions (D'Zurilla \& Goldfried, 1971). The finding that individuals in the written exposure with rescripting condition experienced improvements in negative problem orientation may be indication that they were indeed able to concretize their feared scenario. However it should be noted that in the current study, scripts were not explicitly assessed for concreteness, therefore this suggestions is speculative. Another way to determine whether participants in the exposure conditions were engaging in concrete writing is through examination of SAM ratings. That is, if concrete processing is thought to be associated with reductions in emotional reactivity, then participants in the exposure conditions should demonstrate this between sessions. Supporting this claim, posthoc analysis of SAM ratings showed that participants in both exposure conditions reported decreased emotional reactivity between the first and last writing sessions. Specifically, participants in the worst-case scenario exposure condition showed significant reductions in mean 
arousal from the first to last writing session, $F(1,63)=3.90 p<.05$. Participants in the written exposure with rescripting condition showed significant reductions in mean arousal and mean negative affect from the first to last writing session, $F(1,63)=18.10 p<.01$, and $F(1,63)=$ $22.89 p<.01$, respectively. In addition, participants in both exposure conditions reported less fear of emotional reactivity (i.e., less fear of emotions) following their assigned interventions, which suggests that concrete processing may also counteract fear of emotional reactivity. In summary, both exposure conditions may have allowed individuals to better concretize their worst fear, thereby promoting emotional processing, improvements in fear of emotion, or generation of solutions to hypothetical problems.

\section{Strengths and Limitations}

This study was designed as an extension of prior research on written exposure for GAD, and had several strengths. First, the study comprised a sample of individuals with a principal diagnosis of GAD, and thus findings are relevant to clinical samples. Second, multilevel models were used to examine both the short-term and longer-term (1-month) effects of the interventions, allowing modeling of the stability of the interventions over a longer time period compared to prior work on written exposure for worry and GAD. Third, prior studies on written exposure for GAD and worry did not include negative problem orientation and fear of emotion as outcomes, despite an abundance of research on their relationship to worry and GAD. Collection of this new information allowed for extending the understanding of the written exposure interventions' impact. In addition, administering outcome measures at various time points allowed for examination of potential mechanisms by which the exposure interventions produce change over time. Finally, this was the first known study to (1) adapt rescripting for individuals with GAD and (2) provide preliminary information pointing to the potential of written exposure enhanced 
with rescripting.

The current study also had a number of limitations. First, the study was underpowered, and thus, the sample may have been too small to detect true effects or between-group differences on measures of worry and associated difficulties. In addition, it may have been difficult to detect differences between exposure conditions because the writing interventions were too similar. For example, the first writing session and part of the second writing session were identical in both exposure conditions. In addition, even though only participants in the written exposure with rescripting condition had the added instructions to write about how to move forward from and change their attitude towards their worst fear, some individuals in the worst-case scenario exposure condition also spontaneously reappraised their worst fear, making it difficult to parse out differences between conditions. Finally, whereas studies in other clinical populations that combine exposure with rescripting have shown that one to three sessions of exposure prior to rescripting can be efficacious (e.g., Arntz et al., 2007; Grunert et al., 2007), perhaps for individuals with GAD, the "dose" of exposure prior to rescripting was inadequate and may be viewed as a limitation. However, this was a proof of concept study designed to assess the efficacy of enhancing exposure with rescripting for GAD, and is an important first step to uncovering its potential for improving worry and related cognitive features, as well as informing future studies.

\section{Future Directions}

The present, proof of concept study is the first to demonstrate that written exposure enhanced with rescripting can improve worry and other GAD-related cognitive processes, including negative problem orientation, fear of emotions, perceptions of costliness of the feared scenario, and perceptions of ability to cope with the feared scenario. However, the research on 
written exposure for GAD is still in its infancy; recommendations for future research directions are offered below.

First, little remains known on the optimal parameters of the written exposure intervention in GAD. Parameters including frequency, duration, and spacing of exposure sessions are in need of further investigation in GAD. For example, in the current study, participants in the written exposure with rescripting condition received only half the "dose" of exposure to the worst-case scenario compared to participants in the worst-case scenario exposure condition. Thus, future studies can extend the number or duration of sessions of written exposure to the worst-case scenario prior to rescripting, and examine the degree to which worry and GAD-symptoms improve. This type of research is important for informing empirically supported treatment guidelines for GAD.

Another important future research direction is uncovering whether individuals with GAD may be engaging in anxiety control strategies during written exposure to limit their experience of the feared scenario. Some of the null findings (e.g., lack of change on cognitive avoidance) in the present study indicate this is a possibility. For example, it is possible that individuals with GAD use discrete strategies in an attempt to manage discomfort associated with writing about a feared scenario. In line with this, imaginal exposure treatment protocols suggest that people do engage in anxiety control strategies (i.e., strategies to control unpleasant thoughts, images and emotions), but little is known about their nature and impact on treatment outcome (Koerner \& Fracalanza, 2012). In the current study, all participants wrote about topics for which they indicated a worry rating of 9 or 10 , on a scale of 0 "no worry" to 10 "extreme worry." Thus, participants may have been especially motivated to engage in anxiety control strategies. Dugas and Robichaud (2007) note that anxiety control strategies during imaginal exposure may be 
apparent in the content of individuals' worry scripts. For example, individuals may avoid using details or emotional words in scripts, they may write a script without getting to the worst possible outcome, or they may write the feared scenario in a very factual or vague way. Further research is required to elucidate the nature and extent of these covert avoidance strategies, motivations for engaging in them, whether they suppress emotional arousal, and their association with treatment outcomes.

For example, further research is required to determine whether anxiety control strategies do indeed suppress emotional arousal. Although the current study included a measure of fear of emotion, an important future direction is a thorough investigation of which emotions become activated during exposure, and which emotions individuals with GAD are trying to suppress. For example, it is widely assumed that individuals with GAD avoid thinking about feared situations because they give rise to uncomfortable and intolerable feelings of anxiety. Yet results of the current study showed this may not be the only emotion that individuals with GAD fear and avoid.

Additionally, research is needed to determine whether sharing worry exposure scripts with the researcher may limit engagement with the writing task. For example, participants in the current study were aware that the researcher would be reading their scripts. With this knowledge in mind, it is possible participants were reluctant to explore their very deepest emotions and thoughts about the worst fear coming true. Research has shown that in writing about a stressful life event, private disclosure (i.e., not sharing one's disclosure writing with a researcher) is associated with less avoidance of the specific stressful event than sharing one's disclosure with the researcher (Radcliffe et al., 2007). Further studies can determine whether sharing worry exposure scripts with the researcher motivates cognitive avoidance. 
Another imperative future research direction is the further development and evaluation of a rescripting intervention for GAD. Despite a growing interest in imagery rescripting and its effectiveness in treating other disorders (for a review, see Morina, Lancee \& Arntz, 2017), no known studies have yet applied it to individuals with GAD, presumably because of the complexities in applying it to this population. The imagery rescripting literature posits that the best way to modify negative cognition is by eliciting new positive images to transform and counteract intrusive negative imagery (Holmes et al., 2007). Yet a recent study showed that individuals with GAD, compared to healthy controls, demonstrate less ability to engage in concrete, image-based future thinking, especially if the hypothetical event is positive (Wu, Szpunar, Godovich, Schachter \& Hoffman, 2014). This suggests that one way to optimize the rescripting intervention is to train individuals with GAD on how to engage in concrete and detailed positive future imagery. In fact, some treatment protocols recommend imagery training as an initial step prior to exposure (e.g., Craske et al., 1992). Future research could therefore examine whether the addition of imagery training prior to exposure is associated with improvements in treatment outcome. Imagery ability has also been shown to be associated with larger improvements within exposure-based treatments (e.g., McEvoy, Erceg-Hurn, Saulsman \& Thibodeau, 2015; Mota et al., 2015), therefore, future studies assessing the efficacy of imagery rescripting for GAD would also benefit from the inclusion of a measure of imagery quality as a moderator of outcomes.

Finally, an aspect of the written exposure procedure that requires further investigation is what the exposure target ought to be. Although the conventional way of conducting written exposure is to have people imagine and write about their worst fear coming true, a newer model of GAD, the Contrast Avoidance Model, proposes a different exposure target. According to this 
model, individuals with GAD use worry as a coping strategy to maintain a negative emotional state so that they can avoid an unexpected negative shift in emotion (that is, a negative emotional contrast) and be emotionally prepared for the worst possible outcome (Newman \& Llera, 2011). Therefore, it may be more potent and effective to expose people to mental images of unexpected feared events, because such events are more emotionally evocative than events that are just negative (Newman \& Llera, 2011; Sexton \& Dugas, 2009). Thus, future research could examine whether the written exposure interventions can be used to elicit negative emotional contrast experiences. This differs from prior studies of imaginal exposure and treatment manuals for GAD, where the exposure target has been mental imagery of feared scenarios and the anxious arousal that accompanies this imagery.

Given the dearth of research on both the written exposure and rescripting interventions for GAD, there are several other avenues for future research. For example, the underlying mechanisms of these interventions remain largely unknown. Results of the current study indicate that both interventions are effective for reducing worry and GAD-related cognitive difficulties, but they may work via different pathways. Future research should address the limitations discussed above and continue to investigate cognitive variables that mediate the effects of these interventions.

\section{Conclusions}

This was the first study to adapt imagery rescripting for GAD and examine whether written exposure enhanced with rescripting is more efficacious in reducing worry and related cognitive difficulties than repeated exposure to the same worst-case scenario. Although the

present study was unable to establish superiority of written exposure enhanced with rescripting over worst-case scenario exposure, it provided novel insights into the efficacy of the written 
exposure interventions, and has strong potential to lead to treatment innovations. Written exposure is a promising strategy for GAD, but little is known about how best to conduct it with people with GAD, its mechanisms, and the degree to which the benefits endure beyond one month. Further research is required to address the unanswered questions and pave way for continued improvement of the written exposure interventions, and ultimately lead to advances in GAD treatment. 
Figure 1. Example of the Catastrophizing Interview

\section{Academic Performance}

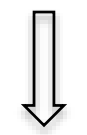

Experimenter: "What is it that worries you about academic performance?"

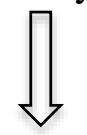

Participant: "Not getting good grades"

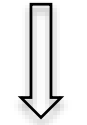

Experimenter: "What would be so bad about not getting good grades?"

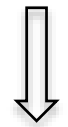

Participant: "I will not get into graduate school."

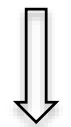

Experimenter: "What would be so bad about not getting into graduate school?"

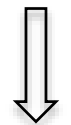

Participant: "I will not be able to pursue the career I want."

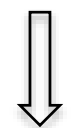

Experimenter: "What would be so bad about not pursuing the career you want?"

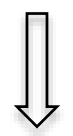

Participant: "I will be a failure."

(stop probing when worst-case scenario is identified) 
Table 1

Sample Characteristics by Condition

$$
\operatorname{RWE}(n=24) \quad \mathrm{WE}(n=23) \quad \mathrm{NC}(n=20)
$$

\begin{tabular}{|c|c|c|c|}
\hline Mean Age in years (SD) & $24.92(5.99)$ & $28.23(6.75)$ & $25.05(5.02)$ \\
\hline \multicolumn{4}{|l|}{ Sex - Frequency $(\%)^{\mathrm{a}}$} \\
\hline Male & $3(12.5 \%)$ & $5(21.7 \%)$ & $2(10 \%)$ \\
\hline Female & $21(87.5 \%)$ & $17(77.3 \%)$ & $18(90 \%)$ \\
\hline \multicolumn{4}{|l|}{ Race/Ethnicity - Frequency (\%) } \\
\hline White & $6(26.1 \%)$ & $15(65.2 \%)$ & $6(31.6 \%)$ \\
\hline East Asian & $3(13 \%)$ & $2(8.7 \%)$ & $2(10.5 \%)$ \\
\hline South Asian & $3(13 \%)$ & $1(4.3 \%)$ & $2(10.5 \%)$ \\
\hline Mixed & $3(13 \%)$ & $1(4.3 \%)$ & $2(10.5)$ \\
\hline South East Asian & $4(16.7 \%)$ & $0(0 \%)$ & $2(10.5 \%)$ \\
\hline Latin American & $1(4.3 \%)$ & $3(13 \%)$ & $1(5.3 \%)$ \\
\hline Black & $1(4.3 \%)$ & $0(0 \%)$ & $2(10.5 \%)$ \\
\hline Arab/West Asian & $1(4.3 \%)$ & $1(4.3 \%)$ & $1(5.0 \%)$ \\
\hline Other & $2(8.7 \%)$ & $0(0 \%)$ & $2(10.5 \%)$ \\
\hline \multicolumn{4}{|l|}{ Marital Status - Frequency $(\%)^{\mathrm{b}}$} \\
\hline Married/Common Law & $3(12.5 \%)$ & $5(21.7 \%)$ & $4(22.2 \%)$ \\
\hline Single & $20(83.3 \%)$ & $15(65.2 \%)$ & $14(77.8 \%)$ \\
\hline Divorced/Widowed & $1(4.2 \%)$ & $3(13 \%)$ & $0(0 \%)$ \\
\hline \multicolumn{4}{|c|}{ Type of Education - Frequency (\%) } \\
\hline College & $2(15.4 \%)$ & $1(10 \%)$ & $0(0 \%)$ \\
\hline University & $11(84.6 \%)$ & $7(70 \%)$ & $10(90.9 \%)$ \\
\hline Adult Education & $0(0 \%)$ & $1(10 \%)$ & $1(9.1 \%)$ \\
\hline Not Applicable & $0(0 \%)$ & $1(10 \%)$ & $0(0 \%)$ \\
\hline \multicolumn{4}{|c|}{ Highest Education - Frequency $(\%)^{\mathfrak{c}}$} \\
\hline High School Diploma & $0(0 \%)$ & $0(0 \%)$ & $0(0 \%)$ \\
\hline College Diploma & $0(0 \%)$ & $2(15.4 \%)$ & $0(0 \%)$ \\
\hline Undergraduate Degree & $7(53.8 \%)$ & $5(38.5 \%)$ & $3(37.5 \%)$ \\
\hline Master's Degree & $1(7.7 \%)$ & $4(30.8 \%)$ & $2(25 \%)$ \\
\hline Doctoral Degree & $3(23.1 \%)$ & $2(15.4 \%)$ & $3(37.5 \%)$ \\
\hline
\end{tabular}




\begin{tabular}{cccc}
\hline & RWE $(n=24)$ & WE $(n=23)$ & NC $(n=20)$ \\
\hline Employment Status - Frequency $(\%)^{\mathrm{d}}$ & & & \\
Unemployed & $8(34.8 \%)$ & $8(34.8 \%)$ & $7(36.8 \%)$ \\
Employed - full time & $4(17.4 \%)$ & $11(47.8 \%)$ & $7(36.8 \%)$ \\
Employed - part-time & $11(47.8 \%)$ & $4(17.4 \%)$ & $5(26.3 \%)$ \\
DSM-5 Diagnoses - Frequency (\%) & & & \\
GAD & $17(70.8 \%)$ & $17(73.9 \%)$ & $12(60 \%)$ \\
Comorbid Mood Disorder & $2(8.3 \%)$ & $2(8.7 \%)$ & $4(20 \%)$ \\
Comorbid Anxiety Disorder & $3(12.5 \%)$ & $3(13.04 \%)$ & $3(15 \%)$ \\
Other Comorbid Disorder ${ }^{\mathrm{a}}$ & $2(8.3 \%)$ & $1(4.3 \%)$ & $1(5 \%)$ \\
\hline
\end{tabular}

Note. $\mathrm{RWE}=$ Rescripted Written Exposure. $\mathrm{WE}=$ Worst Case Scenario Exposure. $\mathrm{NC}=$ Neutral Control. $\mathrm{GAD}=$ Generalized Anxiety Disorder.

${ }^{a}$ One participant in the WE condition chose not to report sex

${ }^{\mathrm{b}}$ Two participants in the NC condition chose not to report marital status

${ }^{\mathrm{c}}$ One participant in the NC condition chose not to report highest education

${ }^{\mathrm{d}}$ One participant in the WE condition chose not to report employment status 
Table 2

Self-Assessment Manikin (SAM) Ratings Separated by Condition

$$
\operatorname{RWE}(n=24) \quad \mathrm{WE}(n=23) \quad \mathrm{NC}(n=20)
$$

Baseline

$\begin{array}{llll}\text { Arousal } & 4.96(2.03) & 4.95(1.88) & 4.10(1.86) \\ \text { Affect } & 4.34(1.31) & 4.10(1.34) & 4.15(1.04)\end{array}$

Writing Session 1

$\begin{array}{llll}\text { Peak Arousal }^{\mathrm{a}} & 6.17(1.92) & 5.76(2.18) & 4.60(1.98) \\ \text { Peak Affect }^{\mathrm{b}} & 6.50(1.93) & 6.52(1.50) & 4.80(1.64)\end{array}$

Writing Session 2

$\begin{array}{llll}\text { Peak Arousal }^{\mathrm{a}} & 4.33(2.42) & 5.47(2.33) & 3.45(1.46) \\ \text { Peak Affect }^{\mathrm{b}} & 5.08(1.91) & 6.05(1.77) & 4.55(1.43)\end{array}$

Writing Session 3

$\begin{array}{llll}\text { Peak Arousal }^{\mathrm{a}} & 4.29(2.25) & 5.00(2.41) & 3.80(1.64) \\ \text { Peak Affect }^{\mathrm{b}} & 4.62(2.06) & 5.95(1.86) & 5.15(1.49)\end{array}$

Note. $\mathrm{RWE}=$ Rescripted Written Exposure. $\mathrm{WE}=$ Worst Case Scenario Exposure $. \mathrm{NC}=$ Neutral Control.

${ }^{\text {a }}$ Peak arousal was defined as the highest of the SAM arousal ratings following baseline

${ }^{\mathrm{b}}$ Peak affect was defined as the highest of the SAM negative affect ratings following baseline 
Table 3

Study 1- Means and Standard Deviations for Measures of Symptoms at Baseline, Visit 3, 1-Week, and 1-month Follow-up by

Condition

\begin{tabular}{|c|c|c|c|c|c|c|}
\hline \multirow[t]{2}{*}{ Measure } & RWE $(n=24)$ & Within & $\mathrm{WE}(n=23)$ & Within & $\mathrm{NC}(n=20)$ & Within \\
\hline & & Cohen's $d$ & \multicolumn{3}{|c|}{ Cohen's $d$} & Cohen's $d$ \\
\hline \multicolumn{7}{|l|}{ PSWQ } \\
\hline Baseline & $68.33(5.35)$ & - & $69.96(4.34)$ & - & $69.10(5.26)$ & - \\
\hline Visit 3 & 64.37 (11.59) & 0.47 & $67.09(6.58)$ & 0.53 & $66.30(7.79)$ & 0.43 \\
\hline 1-week & $63.94(9.32)$ & 0.60 & $65.09(5.59)$ & 0.98 & $63.96(12.78)$ & 0.57 \\
\hline 1-month & $62.95(10.27)$ & 0.69 & $63.10(10.81)$ & 0.91 & $65.20(7.76)$ & 0.60 \\
\hline \multicolumn{7}{|l|}{ PSWQ-PW } \\
\hline Baseline & 67.73 (11.97) & - & $66.91(9.14)$ & - & $66.85(10.10)$ & - \\
\hline Visit 3 & 63.25 (15.09) & 0.33 & $66.74(12.47)$ & 0.02 & $67.15(13.52)$ & -0.03 \\
\hline 1-week & $61.89(15.43)$ & 0.43 & $61.00(10.30)$ & 0.61 & $61.86(12.43)$ & 0.44 \\
\hline 1-month & $62.60(16.61)$ & 0.36 & $59.30(13.05)$ & 0.69 & $58.24(13.38)$ & 0.73 \\
\hline \multicolumn{7}{|l|}{ GAD-Q-IV } \\
\hline Baseline & $9.23(2.59)$ & - & 9.55 (1.72) & - & 9.70 (2.94) & - \\
\hline Visit 3 & $8.78(2.82)$ & 0.17 & 9.44 (1.92) & 0.06 & 8.98 (3.72) & 0.22 \\
\hline 1-week & $8.90(2.52)$ & 0.13 & 9.57 (1.97) & -0.01 & $8.91(3.51)$ & 0.24 \\
\hline 1-month & $8.80(2.85)$ & 0.16 & $8.96(2.23)$ & 0.30 & $8.06(3.83)$ & 0.48 \\
\hline
\end{tabular}




\begin{tabular}{|c|c|c|c|c|c|c|}
\hline Measure & RWE $(n=24)$ & Within & $\mathrm{WE}(n=23)$ & Within & $\mathrm{NC}(n=20)$ & Within \\
\hline & & Cohen's $d$ & & Cohen's $d$ & & Cohen's $d$ \\
\hline \multicolumn{7}{|l|}{ IUS } \\
\hline Baseline & $89.38(18.03)$ & - & $89.91(15.16)$ & - & $88.05(23.72)$ & - \\
\hline Visit 3 & $90.98(22.02)$ & -0.08 & $89.17(14.34)$ & 0.05 & $85.00(21.25)$ & 0.14 \\
\hline 1-week & $88.82(23.75)$ & 0.03 & $86.59(19.31)$ & 0.19 & $84.29(21.74)$ & 0.17 \\
\hline 1-month & $86.81(22.80)$ & 0.13 & $90.40(15.96)$ & -0.03 & $78.46(23.49)$ & 0.41 \\
\hline \multicolumn{7}{|l|}{ CAQ } \\
\hline Baseline & $70.25(16.46)$ & - & $70.86(17.11)$ & - & $73.80(27.58)$ & - \\
\hline Visit 3 & $67.91(19.64)$ & 0.13 & $72.59(16.71)$ & -0.10 & $75.80(22.63)$ & -0.08 \\
\hline 1-week & $65.22(19.94)$ & 0.23 & $66.00(17.86)$ & 0.28 & $78.12(26.85)$ & -0.16 \\
\hline 1-month & $65.30(18.37)$ & 0.28 & $68.45(17.38)$ & 0.14 & $71.54(30.79)$ & 0.08 \\
\hline \multicolumn{7}{|l|}{ NPOQ } \\
\hline Baseline & $42.38(7.60)$ & - & $38.48(8.91)$ & - & 38.65 (13.39) & - \\
\hline Visit 3 & $39.62(9.56)$ & 0.32 & $38.17(9.45)$ & 0.03 & $39.50(11.25)$ & -0.07 \\
\hline 1-week & $40.61(10.46)$ & 0.20 & $38.63(9.90)$ & -0.02 & $40.53(11.46)$ & -0.15 \\
\hline 1-month & $39.95(9.82)$ & 0.28 & $39.54(8.06)$ & -0.12 & 36.85 (13.76) & 0.13 \\
\hline \multicolumn{7}{|l|}{ ACS } \\
\hline Baseline & $166.51(30.39)$ & - & $165.15(20.52)$ & - & $162.40(34.85)$ & - \\
\hline Visit 3 & $159.50(36.72)$ & 0.21 & $158.94(21.26)$ & 0.30 & $162.15(34.32)$ & 0.007 \\
\hline 1-week & $157.78(40.46)$ & 0.25 & $156.27(22.41)$ & 0.41 & $162.18(38.61)$ & 0.006 \\
\hline 1-month & $157.40(23.73)$ & 0.40 & $151.81(43.72)$ & 0.35 & $154.33(35.57)$ & 0.23 \\
\hline
\end{tabular}




\begin{tabular}{|c|c|c|c|c|c|c|}
\hline \multirow[t]{2}{*}{ Measure } & $\operatorname{RWE}(n=24)$ & Within & $\mathrm{WE}(n=23)$ & Within & $\mathrm{NC}(n=20)$ & Within \\
\hline & & Cohen's $d$ & \multicolumn{3}{|c|}{ Cohen's $d$} & Cohen's $d$ \\
\hline \multicolumn{7}{|l|}{ ACS Anxiety } \\
\hline Baseline & $55.27(12.11)$ & - & $60.80(8.17)$ & - & $55.75(11.74)$ & - \\
\hline Visit 3 & $55.21(11.42)$ & 0.005 & $59.65(7.12)$ & 0.15 & $55.40(12.81)$ & 0.03 \\
\hline 1-week & $53.65(14.31)$ & 0.12 & $58.73(7.98)$ & 0.26 & $57.12(14.72)$ & -0.10 \\
\hline 1-month & $52.00(15.22)$ & 0.24 & $60.28(6.80)$ & 0.07 & $56.15(14.35)$ & -0.03 \\
\hline \multicolumn{7}{|l|}{ STICSA-S } \\
\hline Baseline & $45.33(10.58)$ & - & $41.02(9.94)$ & - & 43.15 (14.17) & - \\
\hline Visit 3 & $43.04(13.15)$ & 0.19 & $41.00(8.99)$ & 0.002 & $42.27(11.69)$ & 0.08 \\
\hline 1-week & $41.48(13.11)$ & 0.33 & $38.86(10.46)$ & 0.21 & $41.71(13.09)$ & 0.13 \\
\hline 1-month & $41.55(15.01)$ & 0.30 & $39.00(11.96)$ & 0.18 & $37.38(11.75)$ & 0.53 \\
\hline \multicolumn{7}{|l|}{ STICSA-T } \\
\hline Baseline & $48.67(9.20)$ & - & $45.65(9.87)$ & - & $47.50(13.27)$ & - \\
\hline Visit 3 & $48.54(10.45)$ & 0.01 & $43.69(7.55)$ & 0.22 & $47.00(10.65)$ & 0.04 \\
\hline 1-week & $46.08(13.06)$ & 0.23 & $42.45(9.51)$ & 0.33 & $45.23(13.15)$ & 0.17 \\
\hline 1-month & $46.75(13.49)$ & 0.17 & $43.60(8.14)$ & 0.23 & $40.61(11.37)$ & 0.56 \\
\hline \multicolumn{7}{|l|}{ CES-D-R } \\
\hline Baseline & $27.16(8.07)$ & - & $26.10(5.38)$ & - & $26.06(9.62)$ & - \\
\hline Visit 3 & $26.26(8.85)$ & 0.11 & $22.54(6.68)$ & 0.59 & $27.10(8.06)$ & -0.12 \\
\hline
\end{tabular}




\begin{tabular}{|c|c|c|c|c|c|c|}
\hline Measure & RWE $(n=24)$ & Within & $\mathrm{WE}(n=23)$ & Within & $\mathrm{NC}(n=20)$ & Within \\
\hline & & Cohen's $d$ & & Cohen's $d$ & & Cohen's $d$ \\
\hline 1-week & $24.21(8.14)$ & 0.36 & $20.77(6.14)$ & 0.93 & $24.59(8.31)$ & 0.16 \\
\hline 1-month & $26.25(9.53)$ & 0.10 & $26.40(6.74)$ & -0.05 & $22.77(7.44)$ & 0.39 \\
\hline \multicolumn{7}{|l|}{ Probability } \\
\hline Baseline & $2.92(1.74)$ & - & $3.09(1.68)$ & - & $3.20(1.70)$ & - \\
\hline Visit 3 & $3.21(1.32)$ & -0.19 & $3.33(1.27)$ & -0.16 & $3.29(1.82)$ & -0.05 \\
\hline 1-week & $3.17(1.07)$ & -0.18 & $3.12(1.33)$ & -0.24 & $3.12(1.61)$ & 0.05 \\
\hline 1-month & 3.09 (1.66) & -0.10 & $2.57(0.99)$ & 0.27 & $3.45(1.78)$ & 0.36 \\
\hline \multicolumn{7}{|l|}{ Cost } \\
\hline Baseline & $5.08(1.10)$ & - & $5.00(1.21)$ & - & $5.20(0.89)$ & - \\
\hline Visit 3 & $4.54(1.10)$ & 0.35 & $4.79(1.06)$ & 0.13 & $5.19(1.21)$ & 0.007 \\
\hline 1-week & $4.78(1.13)$ & 0.19 & $5.00(0.93)$ & 0 & $5.24(1.09)$ & -0.03 \\
\hline 1-month & $4.43(1.83)$ & 0.31 & 5.05 (1.09) & -0.03 & $4.57(1.65)$ & 0.35 \\
\hline \multicolumn{7}{|l|}{ Coping } \\
\hline Baseline & $2.17(1.52)$ & - & $2.56(1.56)$ & - & $2.65(1.69)$ & - \\
\hline Visit 3 & $3.08(1.72)$ & -0.40 & 2.78 (1.69) & -0.23 & 2.29 (1.79) & 0.15 \\
\hline 1-week & $2.91(1.65)$ & -0.33 & $2.68(1.58)$ & -0.05 & $3.00(2.06)$ & -0.13 \\
\hline 1-month & $2.50(1.84)$ & -0.14 & $2.60(1.60)$ & -0.02 & $2.50(1.69)$ & 0.06 \\
\hline
\end{tabular}

Note. $\mathrm{RWE}=$ Rescripted Written Exposure. $\mathrm{WE}=$ Worst Case Scenario Exposure $. \mathrm{NC}=$ Neutral Control . PSWQ = Penn State Worry Questionnaire (Meyer, Miller, Metzger, \& Borkovec, 1990); PSWQ-PW = Penn State Worry Questionnaire Past-Week (Stöber \& Bittencourt, 1998); GADQ-IV = Generalized Anxiety Disorder Questionnaire for DSM-IV (GAD-Q-IV; Newman et al., 2002); IUS = Intolerance of Uncertainty Scale (Freeston et al., 1994; English translation, Buhr \& Dugas, 2002); CAQ = Cognitive Avoidance 
Questionnaire (Sexton \& Dugas, 2008); NPOQ = Negative Problem Orientation Questionnaire (Gosselin, Pelletier, \& Ladouceur, 2001; Robichaud \& Dugas, 2005); ACS = Affective Control Scale (Williams, Chambless, \& Ahrens, 1997); ACS Anxiety = Affective Control Scale, Fear of Anxiety Subscale; STICSA-S = State-Trait Inventory for Cognitive and Somatic Anxiety, State Scale (Ree, French, MacLeod, \& Locke, 2000); STICSA-T = State-Trait Inventory for Cognitive and Somatic Anxiety, Trait Scale (Ree, French, MacLeod, \& Locke, 2000); CESD-R = Center for Epidemiologic Studies Depression Scale-Revised (Eaton, Muntaner, Smith, Tien, $\&$ Ybarra, 2004); Probability = Perceived Probability Questions (Berenbaum et al., 2007; Butler \& Mathews, 1983); Cost = Perceived Cost Questions (Berenbaum et al., 2007; Butler \& Mathews, 1983); Coping = Perceived Coping Questions (Berenbaum et al., 2007; Butler \& Mathews, 1983). Within Cohen's $d=$ within-group Cohen's $d$ value representing the magnitude of the change in scores from Baseline to each time point. 
Table 4

Multilevel Models and Piecewise Analyses for PSWQ as Associated with Time and Condition, RWE vs. Neutral

\begin{tabular}{|c|c|c|c|}
\hline & $b$ & $S E$ & $t$ \\
\hline \multicolumn{4}{|l|}{ Total Model Analyses } \\
\hline Intercept & 69.03 & 1.69 & $40.73 * *$ \\
\hline Time & -1.61 & 0.63 & $-2.56^{*}$ \\
\hline Condition & 0.53 & 2.54 & 0.21 \\
\hline Time $\mathrm{x}$ Condition & 0.23 & 0.96 & 0.23 \\
\hline \multicolumn{4}{|l|}{ Contrasts } \\
\hline RWE & -1.61 & 0.63 & $-2.56 *$ \\
\hline Neutral & -1.38 & 0.73 & -1.90 \\
\hline \multicolumn{4}{|l|}{ Piecewise Analyses } \\
\hline Intercept & 72.11 & 2.85 & $25.31 * *$ \\
\hline Time 1 & -3.78 & 1.50 & $-2.52 *$ \\
\hline Time 2 & -0.60 & 0.83 & -0.73 \\
\hline Condition & 0.54 & 4.23 & 0.13 \\
\hline Time 1 x Condition & 0.23 & 2.23 & 0.10 \\
\hline Time $2 \mathrm{x}$ Condition & 0.24 & 1.27 & 0.19 \\
\hline \multicolumn{4}{|l|}{ Time 1 Contrasts } \\
\hline RWE & -3.78 & 1.50 & $-2.52 *$ \\
\hline Neutral & -3.55 & 1.65 & $-2.15^{*}$ \\
\hline \multicolumn{4}{|l|}{ Time 2 Contrasts } \\
\hline RWE & -0.60 & 0.83 & -0.73 \\
\hline Neutral & -0.36 & 0.96 & -0.38 \\
\hline
\end{tabular}

Note. $\mathrm{RWE}=$ Rescripted Written Exposure. $\mathrm{NC}=$ Neutral Control. $\mathrm{PSWQ}=$ Penn State Worry Questionnaire (Meyer, Miller, Metzger, \& Borkovec, 1990).

Under Contrasts, $R W E$ represents within-group changes in the RWE condition, and Neutral represents within-group changes in the control condition.

Under Piecewise Analyses, Time 1= Intervention period, during which writing sessions 1 to 3 occurred (i.e., Visits 1-3); Time 2= Follow-up period (i.e., Visits 3 to 5)

$* p<.05 ; * * p<.01$ 
Table 5

Multilevel Models and Piecewise Analyses for PSWQ as Associated with Time and Condition, WE vs. Neutral

\begin{tabular}{|c|c|c|c|}
\hline & $b$ & $S E$ & $t$ \\
\hline \multicolumn{4}{|l|}{ Total Model Analyses } \\
\hline Intercept & 72.07 & 1.35 & $53.51 * *$ \\
\hline Time & -2.32 & 0.65 & $-3.55 * *$ \\
\hline Condition & -2.55 & 2.01 & -1.23 \\
\hline Time $\mathrm{x}$ Condition & 0.96 & 0.99 & 0.96 \\
\hline \multicolumn{4}{|l|}{ Contrasts } \\
\hline WE & -2.32 & 0.65 & $-3.55^{* *}$ \\
\hline Neutral & -1.38 & 0.74 & -1.83 \\
\hline \multicolumn{4}{|l|}{ Piecewise Analyses } \\
\hline Intercept & 72.76 & 2.45 & $29.64 * *$ \\
\hline Time 1 & -3.60 & 1.52 & $-2.34 * *$ \\
\hline Time 2 & -2.11 & 0.88 & $-2.40 *$ \\
\hline Condition & -0.063 & 3.60 & -.02 \\
\hline Time 1 x Condition & -0.79 & 2.08 & -0.38 \\
\hline Time 2 x Condition & 1.89 & 1.34 & 1.41 \\
\hline \multicolumn{4}{|l|}{ Time 1 Contrasts } \\
\hline WE & -3.60 & 1.52 & $-2.34 *$ \\
\hline Neutral & -2.81 & 1.41 & $-1.99 *$ \\
\hline \multicolumn{4}{|l|}{ Time 2 Contrasts } \\
\hline WE & -2.11 & 0.88 & $-2.40^{*}$ \\
\hline Neutral & -0.21 & 1.01 & -0.21 \\
\hline
\end{tabular}

Note. $\mathrm{WE}=$ Worst-Case Scenario Exposure. $\mathrm{NC}=$ Neutral Control. $\mathrm{PSWQ}=$ Penn State Worry Questionnaire (Meyer, Miller, Metzger, \& Borkovec, 1990).

Under Contrasts, WE represents within-group changes in the WE condition, and Neutral represents within-group changes in the control condition.

Under Piecewise Analyses, Time 1= Intervention period, during which writing sessions 1 to 3 occurred (i.e., Visits 1-3); Time 2= Follow-up period (i.e., Visits 3 to 5)

$* p<.05 ; * * p<.01$ 
Table 6

Multilevel Models and Piecewise Analyses for PSWQ as Associated with Time and Condition, RWE vs. WE

\begin{tabular}{|c|c|c|c|}
\hline & $b$ & $S E$ & $t$ \\
\hline \multicolumn{4}{|l|}{ Total Model Analyses } \\
\hline Intercept & 69.02 & 1.24 & $55.48 * *$ \\
\hline Time & -1.60 & 0.66 & $-2.42 *$ \\
\hline Condition & 3.05 & 1.77 & 1.73 \\
\hline Time $\mathrm{x}$ Condition & -0.72 & 0.94 & -0.76 \\
\hline \multicolumn{4}{|l|}{ Contrasts } \\
\hline RWE & -1.60 & 0.66 & $-2.42 *$ \\
\hline WE & -2.32 & 0.87 & $-3.45^{*}$ \\
\hline \multicolumn{4}{|l|}{ Piecewise Analyses } \\
\hline Intercept & 72.11 & 2.33 & $30.87 * *$ \\
\hline Time 1 & -3.78 & 1.25 & $-3.00 * *$ \\
\hline Time 2 & -0.59 & 0.83 & -0.71 \\
\hline Condition & 0.64 & 3.34 & 0.19 \\
\hline Time $1 \times$ Condition & 0.97 & 1.80 & 0.54 \\
\hline Time $2 \mathrm{x}$ Condition & -1.52 & 1.18 & -1.28 \\
\hline \multicolumn{4}{|l|}{ Time 1 Contrasts } \\
\hline RWE & -3.78 & 1.25 & $-3.00 * *$ \\
\hline WE & -3.60 & 1.52 & $-2.34 *$ \\
\hline \multicolumn{4}{|l|}{ Time 2 Contrasts } \\
\hline RWE & -0.59 & 0.83 & -0.71 \\
\hline WE & -2.10 & 0.84 & $-2.51 *$ \\
\hline
\end{tabular}

Note. RWE $=$ Rescripted Written Exposure. WE = Worst-Case Scenario Exposure. PSWQ = Penn State Worry Questionnaire (Meyer, Miller, Metzger, \& Borkovec, 1990).

Under Contrasts, $R W E$ represents within-group changes in the RWE condition, and $W E$ represents within-group changes in the WE condition.

Under Piecewise Analyses, Time 1= Intervention period, during which writing sessions 1 to 3 occurred (i.e., Visits 1-3); Time 2= Follow-up period (i.e., Visits 3 to 5)

$* p<.05 ; * * p<.01$ 
Table 7

Study 2- Multilevel Models and Piecewise Analyses for GADQ-IV as Associated with Time and Condition, RWE vs. Neutral

\begin{tabular}{|c|c|c|c|}
\hline & $b$ & $S E$ & $t$ \\
\hline \multicolumn{4}{|l|}{ Total Model Analyses } \\
\hline Intercept & 9.10 & 1.12 & $8.10 * *$ \\
\hline Time & -.04 & 0.66 & -.06 \\
\hline Condition & 2.21 & 1.70 & 1.30 \\
\hline Time $\mathrm{x}$ Condition & -1.54 & 0.99 & -1.55 \\
\hline \multicolumn{4}{|l|}{ Contrasts } \\
\hline RWE & -1.58 & 0.75 & $-2.11 *$ \\
\hline Neutral & -.04 & 0.66 & -.06 \\
\hline \multicolumn{4}{|l|}{ Piecewise Analyses } \\
\hline Intercept & 9.69 & 0.75 & $12.87 * *$ \\
\hline Time 1 & -0.46 & 0.31 & -1.51 \\
\hline Time 2 & 0.24 & 2.29 & 0.10 \\
\hline Condition & 0.79 & 1.11 & 0.71 \\
\hline Time 1 x Condition & -0.32 & 0.46 & -0.69 \\
\hline Time $2 \times$ Condition & -4.06 & 3.44 & -1.18 \\
\hline \multicolumn{4}{|l|}{ Time 1 Contrasts } \\
\hline RWE & -0.78 & 0.34 & -2.27 \\
\hline Neutral & -0.46 & 0.31 & -1.51 \\
\hline \multicolumn{4}{|l|}{ Time 2 Contrasts } \\
\hline RWE & -3.82 & 2.57 & -1.49 \\
\hline Neutral & 0.24 & 2.29 & 0.10 \\
\hline
\end{tabular}

Note. $\mathrm{RWE}=$ Rescripted Written Exposure. $\mathrm{NC}=$ Neutral Control. GADQ-IV = Generalized Anxiety Disorder Questionnaire for DSM-IV (GAD-Q-IV; Newman et al., 2002).

Under Contrasts, $R W E$ represents within-group changes in the RWE condition, and Neutral represents within-group changes in the control condition.

Under Piecewise Analyses, Time 1= Intervention period, during which writing sessions 1 to 3 occurred (i.e., Visits 1-3); Time 2= Follow-up period (i.e., Visits 3 to 5)

$* p<.05 ; * * p<.01$ 
Table 8

Multilevel Models and Piecewise Analyses for GADQ-IV as Associated with Time and Condition, WE vs. Neutral

\begin{tabular}{|c|c|c|c|}
\hline & $b$ & $S E$ & $t$ \\
\hline \multicolumn{4}{|l|}{ Total Model Analyses } \\
\hline Intercept & 9.76 & 1.14 & $8.58 * *$ \\
\hline Time & -0.15 & 0.66 & -0.22 \\
\hline Condition & 1.55 & 1.70 & 0.91 \\
\hline Time $\mathrm{x}$ Condition & -1.44 & 0.99 & -1.44 \\
\hline \multicolumn{4}{|l|}{ Contrasts } \\
\hline WE & -1.58 & 0.75 & $-2.12 *$ \\
\hline Neutral & -0.15 & 0.66 & -0.22 \\
\hline \multicolumn{4}{|l|}{ Piecewise Analyses } \\
\hline Intercept & 9.58 & 0.71 & $13.51 * *$ \\
\hline Time 1 & -0.02 & 0.30 & -0.08 \\
\hline Time 2 & -0.19 & 2.36 & -0.08 \\
\hline Condition & 0.89 & 1.03 & 0.86 \\
\hline Time 1 x Condition & -0.75 & 0.44 & -1.69 \\
\hline Time $2 \times$ Condition & -3.63 & 3.52 & -1.03 \\
\hline \multicolumn{4}{|l|}{ Time 1 Contrasts } \\
\hline WE & -0.78 & 0.32 & -2.37 \\
\hline Neutral & -0.02 & 0.30 & -0.08 \\
\hline \multicolumn{4}{|l|}{ Time 2 Contrasts } \\
\hline WE & -3.82 & 2.60 & -1.46 \\
\hline Neutral & -0.19 & 2.36 & -0.08 \\
\hline
\end{tabular}

Note. $\mathrm{WE}=$ Worst Case Scenario Exposure. $\mathrm{NC}=$ Neutral Control. GADQ-IV = Generalized Anxiety Disorder Questionnaire for DSM-IV (GAD-Q-IV; Newman et al., 2002). Under Contrasts, WE represents within-group changes in the WE condition, and Neutral represents within-group changes in the control condition.

Under Piecewise Analyses, Time 1= Intervention period, during which writing sessions 1 to 3 occurred (i.e., Visits 1-3); Time 2= Follow-up period (i.e., Visits 3 to 5)

$* p<.05 ; * * p<.01$ 
Table 9

Multilevel Models and Piecewise Analyses for GADQ-IV as Associated with Time and Condition, RWE vs. WE

$b \quad S E$

\begin{tabular}{lccc}
\hline Total Model Analyses & & & \\
Intercept & 9.14 & 0.49 & $18.30^{* *}$ \\
Time & -.06 & 0.13 & -0.46 \\
Condition & 0.64 & 0.71 & 0.90 \\
Time x Condition & -.09 & 0.19 & -0.51 \\
Contrasts & & & -1.18 \\
RWE & -0.16 & 0.13 & -0.46 \\
WE & -.06 & 0.13 &
\end{tabular}

Piecewise Analyses

$\begin{array}{lccc}\text { Intercept } & 9.65 & 0.63 & 15.23^{* *} \\ {\text { Time } 1^{\mathrm{a}}}^{\mathrm{a}} & -0.42 & 0.28 & -1.51 \\ \text { Time } 2^{\mathrm{b}} & 0.13 & 0.24 & 0.52 \\ \text { Condition } & -0.04 & 0.91 & -0.04 \\ \text { Time 1 x Condition } & 0.28 & 0.41 & 0.35 \\ \text { Time 2 x Condition } & -0.34 & 0.34 & -0.98 \\ \text { me 1 Contrasts } & & & \\ \text { RWE } & -0.42 & 0.28 & -1.51 \\ \text { WE } & -0.04 & 0.29 & -0.14 \\ \text { me 2 Contrasts } & & & 0.52 \\ \text { RWE } & 0.13 & 0.24 & -0.87 \\ \text { WE } & -0.21 & 0.24 & \end{array}$

Note. $\mathrm{RWE}=$ Rescripted Written Exposure. WE = Worst-Case Scenario Exposure. GADQ-IV = Generalized Anxiety Disorder Questionnaire for DSM-IV (GAD-Q-IV; Newman et al., 2002). Under Contrasts, $R W E$ represents within-group changes in the RWE condition, and $W E$ represents within-group changes in the WE condition.

Under Piecewise Analyses, Time 1= Intervention period, during which writing sessions 1 to 3 occurred (i.e., Visits 1-3); Time 2= Follow-up period (i.e., Visits 3 to 5) $* p<.05 ; * * p<.01$ 
Table 10

Multilevel Models and Piecewise Analyses for STICSA-T as Associated with Time and Condition, RWE vs. NC

\begin{tabular}{|c|c|c|c|}
\hline & $b$ & $S E$ & $t$ \\
\hline \multicolumn{4}{|l|}{ Total Model Analyses } \\
\hline Intercept & 49.55 & 2.29 & $21.62 * *$ \\
\hline Time & -0.81 & 0.59 & 0.18 \\
\hline Condition & -1.08 & 3.42 & 0.75 \\
\hline Time $\mathrm{x}$ Condition & -0.13 & 0.92 & -0.15 \\
\hline \multicolumn{4}{|l|}{ Contrasts } \\
\hline RWE & -0.81 & 0.59 & 0.18 \\
\hline $\mathrm{NC}$ & -0.94 & 0.70 & -1.35 \\
\hline \multicolumn{4}{|l|}{ Piecewise Analyses } \\
\hline Intercept & 49.28 & 2.92 & $16.84 * *$ \\
\hline Time 1 & -0.62 & 1.24 & -0.49 \\
\hline Time 2 & -0.91 & 0.92 & -0.99 \\
\hline Condition & -0.92 & 4.34 & -0.21 \\
\hline Time 1 x Condition & -0.25 & 1.85 & -0.13 \\
\hline Time $2 \times$ Condition & -0.08 & 1.43 & -0.06 \\
\hline \multicolumn{4}{|l|}{ Time 1 Contrasts } \\
\hline RWE & -0.62 & 1.24 & -0.49 \\
\hline $\mathrm{NC}$ & -0.86 & 1.37 & -0.63 \\
\hline \multicolumn{4}{|l|}{ Time 2 Contrasts } \\
\hline RWE & -0.91 & 0.92 & -0.99 \\
\hline $\mathrm{NC}$ & -0.99 & 1.09 & -0.90 \\
\hline
\end{tabular}

Note. $\mathrm{RWE}=$ Rescripted Written Exposure. $\mathrm{NC}=$ Neutral Control. STICSA-T $=$ State-Trait Inventory for Cognitive and Somatic Anxiety, Trait Scale (Ree, French, MacLeod, \& Locke, 2000). Under Contrasts, $R W E$ represents within-group changes in the RWE condition, and Neutral represents within-group changes in the control condition. Under Piecewise Analyses, Time 1= Intervention period, during which writing sessions 1 to 3 occurred (i.e., Visits 1-3); Time 2= Follow-up period (i.e., Visits 3 to 5) $* p<.05 ; * * p<.01$ 
Table 11

Multilevel Models and Piecewise Analyses for STICSA-T as Associated with Time and Condition, WE vs. NC

\begin{tabular}{|c|c|c|c|}
\hline & $b$ & $S E$ & $t$ \\
\hline \multicolumn{4}{|l|}{ Total Model Analyses } \\
\hline Intercept & 45.74 & 2.30 & $19.89 * *$ \\
\hline Time & -0.75 & 0.47 & -1.56 \\
\hline Condition & 2.74 & 3.38 & 0.81 \\
\hline Time $\mathrm{x}$ Condition & -0.20 & 0.73 & -0.28 \\
\hline \multicolumn{4}{|l|}{ Contrasts } \\
\hline WE & -0.75 & -0.47 & -1.56 \\
\hline $\mathrm{NC}$ & -0.95 & -0.74 & -1.70 \\
\hline \multicolumn{4}{|l|}{ Piecewise Analyses } \\
\hline Intercept & 47.97 & 2.62 & $18.30 * *$ \\
\hline Time 1 & -2.32 & 1.00 & $-2.31 *$ \\
\hline Time 2 & 0.03 & 0.82 & 0.04 \\
\hline Condition & 0.39 & 3.85 & 0.10 \\
\hline Time $1 \mathrm{x}$ Condition & 1.44 & 1.47 & 0.98 \\
\hline Time $2 \times$ Condition & -0.99 & 1.26 & -0.78 \\
\hline \multicolumn{4}{|l|}{ Time 1 Contrasts } \\
\hline WE & -2.32 & 1.00 & $-2.31 *$ \\
\hline $\mathrm{NC}$ & -0.87 & 1.08 & -0.81 \\
\hline \multicolumn{4}{|l|}{ Time 2 Contrasts } \\
\hline WE & 0.03 & 0.82 & 0.04 \\
\hline $\mathrm{NC}$ & -0.95 & 0.96 & -0.99 \\
\hline
\end{tabular}

Note. WE $=$ Worst-Case Scenario Exposure. $\mathrm{NC}=$ Neutral Control. STICSA-T = State-Trait Inventory for Cognitive and Somatic Anxiety, Trait Scale (Ree, French, MacLeod, \& Locke, 2000). Under Contrasts, WE represents within-group changes in the WE condition, and Neutral represents within-group changes in the control condition. Under Piecewise Analyses, Time 1= Intervention period, during which writing sessions 1 to 3 occurred (i.e., Visits 1-3); Time 2= Follow-up period (i.e., Visits 3 to 5) $* p<.05 ; * * p<.01$ 
Table 12

Multilevel Models and Piecewise Analyses for STICSA-T as Associated with Time and Condition, RWE vs. WE

\begin{tabular}{|c|c|c|c|}
\hline & $b$ & $S E$ & $t$ \\
\hline \multicolumn{4}{|l|}{ Total Model Analyses } \\
\hline Intercept & 49.55 & 1.99 & $24.85^{* *}$ \\
\hline Time & -0.81 & 0.59 & -1.36 \\
\hline Condition & -3.82 & 2.85 & -1.34 \\
\hline Time $\mathrm{x}$ Condition & 0.06 & 0.84 & 0.08 \\
\hline \multicolumn{4}{|l|}{ Contrasts } \\
\hline RWE & -0.73 & 0.60 & -1.32 \\
\hline WE & -0.81 & 0.59 & -1.36 \\
\hline \multicolumn{4}{|l|}{ Piecewise Analyses } \\
\hline Intercept & 49.27 & 2.63 & $18.70 * *$ \\
\hline Time 1 & -0.61 & 1.21 & -0.50 \\
\hline Time 2 & -0.92 & 0.92 & -0.99 \\
\hline Condition & -1.31 & 3.76 & -0.35 \\
\hline Time 1 x Condition & -1.70 & 1.73 & -0.98 \\
\hline Time $2 \times$ Condition & 0.94 & 1.31 & 0.71 \\
\hline \multicolumn{4}{|l|}{ Time 1 Contrasts } \\
\hline RWE & -0.61 & 1.21 & -0.50 \\
\hline WE & -2.31 & 1.23 & -1.87 \\
\hline \multicolumn{4}{|l|}{ Time 2 Contrasts } \\
\hline RWE & 0.01 & 0.93 & 0.01 \\
\hline WE & -0.92 & 0.92 & -0.99 \\
\hline
\end{tabular}

Note. $\mathrm{RWE}=$ Rescripted Written Exposure. WE = Worst-Case Scenario Exposure.

STICSA-T = State-Trait Inventory for Cognitive and Somatic Anxiety, Trait Scale (Ree et al., 2000). Under Contrasts, $R W E$ represents within-group changes in the RWE condition, and WE represents within-group changes in the WE condition.

Under Piecewise Analyses, Time 1= Intervention period, during which writing sessions 1 to 3 occurred (i.e., Visits 1-3); Time 2= Follow-up period (i.e., Visits 3 to 5)

$* p<.05 ; * * p<.01$ 
Table 13

Multilevel Models and Piecewise Analyses for CESD-R as Associated with Time and Condition, RWE vs. NC

\begin{tabular}{|c|c|c|c|}
\hline & $b$ & $S E$ & $t$ \\
\hline \multicolumn{4}{|l|}{ Total Model Analyses } \\
\hline Intercept & 27.45 & 1.84 & $14.90 * *$ \\
\hline Time & -0.63 & 0.51 & -1.24 \\
\hline Condition & 3.93 & 2.75 & 1.42 \\
\hline Time $\mathrm{x}$ Condition & -1.32 & 0.79 & -1.67 \\
\hline \multicolumn{4}{|l|}{ Contrasts } \\
\hline RWE & -0.63 & 0.51 & -1.24 \\
\hline $\mathrm{NC}$ & -0.99 & 0.60 & -1.32 \\
\hline \multicolumn{4}{|l|}{ Piecewise Analyses } \\
\hline Intercept & 28.61 & 2.41 & $11.88 * *$ \\
\hline Time 1 & -1.44 & 1.16 & -1.24 \\
\hline Time 2 & -0.24 & 0.85 & -0.28 \\
\hline Condition & 4.92 & 3.57 & 1.38 \\
\hline Time 1 x Condition & -2.03 & 1.73 & -1.17 \\
\hline Time $2 \times$ Condition & -0.95 & 1.33 & -0.71 \\
\hline \multicolumn{4}{|l|}{ Time 1 Contrasts } \\
\hline RWE & -1.44 & 1.16 & -1.24 \\
\hline $\mathrm{NC}$ & -2.02 & 1.74 & -1.16 \\
\hline \multicolumn{4}{|l|}{ Time 2 Contrasts } \\
\hline RWE & -0.24 & 0.85 & -0.28 \\
\hline $\mathrm{NC}$ & -1.19 & 1.02 & -1.16 \\
\hline
\end{tabular}

Note. $\mathrm{RWE}=$ Rescripted Written Exposure. $\mathrm{NC}=$ Neutral Control. $\mathrm{CESD}-\mathrm{R}=$ Center for Epidemiologic Studies Depression Scale-Revised (Eaton et al., 2004).

Under Contrasts, $R W E$ represents within-group changes in the RWE condition, and Neutral represents within-group changes in the control condition.

Under Piecewise Analyses, Time 1= Intervention period, during which writing sessions 1 to 3 occurred (i.e., Visits 1-3); Time 2= Follow-up period (i.e., Visits 3 to 5)

$* p<.05 ; * * p<.01$ 
Table 14

Multilevel Models and Piecewise Analyses for CESD-R as Associated with Time and Condition, WE vs. NC

\begin{tabular}{|c|c|c|c|}
\hline & $b$ & $S E$ & $t$ \\
\hline \multicolumn{4}{|l|}{ Total Model Analyses } \\
\hline Intercept & 22.32 & 1.62 & $13.76^{* *}$ \\
\hline Time & -0.17 & 0.43 & -0.39 \\
\hline Condition & 9.07 & 2.39 & 3.78 \\
\hline Time $\mathrm{x}$ Condition & -1.79 & 0.66 & -2.69 \\
\hline \multicolumn{4}{|l|}{ Contrasts } \\
\hline WE & -0.17 & 0.43 & -0.39 \\
\hline $\mathrm{NC}$ & -1.96 & 0.50 & -3.88 \\
\hline \multicolumn{4}{|l|}{ Piecewise Analyses } \\
\hline Intercept & 22.14 & 2.02 & $10.92 * *$ \\
\hline Time 1 & -0.03 & 0.92 & -0.04 \\
\hline Time 2 & -0.28 & 0.73 & -0.39 \\
\hline Condition & 11.39 & 2.97 & 3.83 \\
\hline Time 1 x Condition & -3.44 & 1.36 & -2.51 \\
\hline Time $2 \times$ Condition & -0.90 & 1.14 & -0.79 \\
\hline \multicolumn{4}{|l|}{ Time 1 Contrasts } \\
\hline WE & -0.03 & 0.92 & -0.04 \\
\hline $\mathrm{NC}$ & -1.47 & 1.00 & -3.45 \\
\hline \multicolumn{4}{|l|}{ Time 2 Contrasts } \\
\hline WE & -0.28 & 0.73 & -0.39 \\
\hline $\mathrm{NC}$ & -1.44 & 1.36 & -2.51 \\
\hline
\end{tabular}

Note. $\mathrm{WE}=$ Worst-Case Scenario Exposure. $\mathrm{NC}=$ Neutral Control. $\mathrm{CESD}-\mathrm{R}=$ Center for Epidemiologic Studies Depression Scale-Revised (Eaton et al., 2004).

Under Contrasts, WE represents within-group changes in the WE condition, and Neutral represents within-group changes in the control condition.

Under Piecewise Analyses, Time 1= Intervention period, during which writing sessions 1 to 3 occurred (i.e., Visits 1-3); Time 2= Follow-up period (i.e., Visits 3 to 5)

$* p<.05 ; * * p<.01$ 
Table 15

Multilevel Models and Piecewise Analyses for CESD-R as Associated with Time and Condition, RWE vs. WE

\begin{tabular}{|c|c|c|c|}
\hline & $b$ & $S E$ & $t$ \\
\hline \multicolumn{4}{|l|}{ Total Model Analyses } \\
\hline Intercept & 27.45 & 1.59 & $17.23^{* *}$ \\
\hline Time & -0.63 & 0.47 & -1.35 \\
\hline Condition & -5.14 & 2.27 & -2.26 \\
\hline Time $\mathrm{x}$ Condition & 0.46 & 0.66 & 0.69 \\
\hline \multicolumn{4}{|l|}{ Contrasts } \\
\hline RWE & -0.63 & 0.47 & -1.35 \\
\hline WE & -0.16 & 0.47 & -0.36 \\
\hline \multicolumn{4}{|l|}{ Piecewise Analyses } \\
\hline Intercept & 28.61 & 2.12 & $13.49 * *$ \\
\hline Time 1 & -1.44 & 1.05 & -1.38 \\
\hline Time 2 & -0.24 & 0.80 & -0.30 \\
\hline Condition & -6.47 & 3.03 & -2.13 \\
\hline Time 1 x Condition & 1.40 & 1.49 & 0.94 \\
\hline Time $2 \mathrm{x}$ Condition & -0.03 & 1.13 & -0.03 \\
\hline \multicolumn{4}{|l|}{ Time 1 Contrasts } \\
\hline RWE & -1.44 & 1.05 & -1.38 \\
\hline WE & -0.04 & 1.06 & -0.04 \\
\hline \multicolumn{4}{|l|}{ Time 2 Contrasts } \\
\hline RWE & -0.24 & 0.80 & -0.30 \\
\hline WE & 1.40 & 1.49 & 0.94 \\
\hline
\end{tabular}

Note. $\mathrm{RWE}=$ Rescripted Written Exposure. $\mathrm{WE}=$ Worst-Case Scenario Exposure.

CESD-R = Center for Epidemiologic Studies Depression Scale-Revised (Eaton et al., 2004). Under Contrasts, $R W E$ represents within-group changes in the RWE condition, and $W E$ represents within-group changes in the WE condition.

Under Piecewise Analyses, Time 1= Intervention period, during which writing sessions 1 to 3 occurred (i.e., Visits 1-3); Time 2= Follow-up period (i.e., Visits 3 to 5)

$* p<.05 ; * * p<.01$ 
Table 16

Multilevel Models and Piecewise Analyses for IUS as Associated with Time and Condition, RWE vs. $N C$

\begin{tabular}{|c|c|c|c|}
\hline & $b$ & $S E$ & $t$ \\
\hline \multicolumn{4}{|l|}{ Total Model Analyses } \\
\hline Intercept & 91.01 & 4.27 & $21.30 * *$ \\
\hline Time & -0.69 & 1.04 & -0.67 \\
\hline Condition & -1.26 & 1.59 & -0.80 \\
\hline \multicolumn{4}{|l|}{ Time $\mathrm{x}$ Condition } \\
\hline \multicolumn{4}{|l|}{ Contrasts } \\
\hline RWE & -0.69 & 1.04 & -0.67 \\
\hline Neutral & -1.98 & 1.03 & -0.67 \\
\hline \multicolumn{4}{|l|}{ Piecewise Analyses } \\
\hline Intercept & 87.83 & 5.54 & $15.84 * *$ \\
\hline Time 1 & 1.55 & 2.35 & 0.66 \\
\hline Time 2 & -1.82 & 1.42 & -1.27 \\
\hline Condition & 2.88 & 8.23 & 0.35 \\
\hline Time $1 \times$ Condition & -4.21 & 3.49 & -1.21 \\
\hline Time $2 \times$ Condition & 0.02 & 2.23 & 0.009 \\
\hline \multicolumn{4}{|l|}{ Time 1 Contrasts } \\
\hline RWE & 1.55 & 2.35 & 0.66 \\
\hline Neutral & -2.66 & 2.58 & -1.03 \\
\hline \multicolumn{4}{|l|}{ Time 2 Contrasts } \\
\hline RWE & -1.82 & 1.42 & -1.27 \\
\hline Neutral & -1.79 & 1.71 & -1.05 \\
\hline
\end{tabular}

Note. $\mathrm{RWE}=$ Rescripted Written Exposure. $\mathrm{NC}=$ Neutral Control. IUS $=$ Intolerance of Uncertainty Scale (Freeston et al., 1994; English translation, Buhr \& Dugas, 2002). Under Contrasts, $R W E$ represents within-group changes in the RWE condition, and Neutral represents within-group changes in the control condition.

Under Piecewise Analyses, Time 1= Intervention period, during which writing sessions 1 to 3 occurred (i.e., Visits 1-3); Time 2= Follow-up period (i.e., Visits 3 to 5)

$* p<.05 ; * * p<.01$ 
Table 17

Multilevel Models and Piecewise Analyses for IUS as Associated with Time and Condition, WE vs. NC

\begin{tabular}{lccc}
\hline & $b$ & $S E$ & $t$ \\
\hline Total Model Analyses & & & \\
Intercept & 90.08 & 4.02 & $22.39 * *$ \\
Time & -0.58 & 0.85 & -0.68 \\
Condition & -0.29 & 5.93 & -0.048 \\
Time x Condition & -1.43 & 1.30 & -1.1 \\
Contrasts & & & \\
WE & -0.58 & 0.85 & -0.68 \\
Neutral & -2.01 & 0.98 & -2.04 \\
Piecewise Analyses & & & \\
Intercept & 91.44 & 4.72 & $19.36 * *$ \\
Time 1 & -1.53 & 1.83 & -0.83 \\
Time 2 & -0.18 & 1.35 & -0.13 \\
Condition & -0.68 & 6.92 & -0.098 \\
Time 1 x Condition & -1.18 & 2.69 & -0.44 \\
Time 2 x Condition & -1.45 & 2.09 & -0.69 \\
Time 1 Contrasts & & & -0.83 \\
WE & -1.53 & 1.83 & -1.37 \\
Neutral & -2.71 & 1.98 & -1.02 \\
Time 2 Contrasts & & & \\
WE & -0.18 & 1.35 & \\
Neutral & -1.63 & & \\
\hline
\end{tabular}

Note. $\mathrm{WE}=$ Worst Case Scenario Exposure. $\mathrm{NC}=$ Neutral Control. $\mathrm{IUS}=$ Intolerance of Uncertainty Scale (Freeston et al., 1994; English translation, Buhr \& Dugas, 2002). Under Contrasts, WE represents within-group changes in the WE condition, and Neutral represents within-group changes in the control condition.

Under Piecewise Analyses, Time 1= Intervention period, during which writing sessions 1 to 3 occurred (i.e., Visits 1-3); Time 2= Follow-up period (i.e., Visits 3 to 5)

$* p<.05 ; * * p<.01$ 
Table 18

Multilevel Models and Piecewise Analyses for IUS as Associated with Time and Condition, RWE vs. WE

\begin{tabular}{|c|c|c|c|}
\hline & $b$ & $S E$ & $t$ \\
\hline \multicolumn{4}{|l|}{ Total Model Analyses } \\
\hline Intercept & 91.00 & 3.51 & 25.90 \\
\hline Time & -0.69 & 1.05 & -0.66 \\
\hline Condition & -0.88 & 5.01 & -0.18 \\
\hline Time $\mathrm{x}$ Condition & 0.09 & 1.49 & 0.06 \\
\hline \multicolumn{4}{|l|}{ Contrasts } \\
\hline RWE & -0.69 & 1.05 & -0.66 \\
\hline WE & -0.59 & 1.06 & -0.56 \\
\hline \multicolumn{4}{|l|}{ Piecewise Analyses } \\
\hline Intercept & 87.85 & 4.93 & $17.82 * *$ \\
\hline Time 1 & 1.53 & 2.32 & 0.66 \\
\hline Time 2 & -1.79 & 1.41 & -1.27 \\
\hline Condition & 3.58 & 7.04 & 0.51 \\
\hline Time 1 x Condition & -3.05 & 3.32 & -0.92 \\
\hline Time $2 \mathrm{x}$ Condition & 1.57 & 2.00 & 0.78 \\
\hline \multicolumn{4}{|l|}{ Time 1 Contrasts } \\
\hline RWE & 1.53 & 2.32 & 0.66 \\
\hline WE & -1.51 & 2.37 & -0.64 \\
\hline \multicolumn{4}{|l|}{ Time 2 Contrasts } \\
\hline RWE & -1.79 & 1.41 & -1.27 \\
\hline WE & -0.22 & 1.42 & -0.15 \\
\hline
\end{tabular}

Note. $\mathrm{RWE}=$ Rescripted Written Exposure. WE = Worst-Case Scenario Exposure. IUS = Intolerance of Uncertainty Scale (Freeston et al., 1994; English translation, Buhr \& Dugas, 2002).

Under Contrasts, $R W E$ represents within-group changes in the RWE condition, and WE represents within-group changes in the WE condition.

Under Piecewise Analyses, Time 1= Intervention period, during which writing sessions 1 to 3 occurred (i.e., Visits 1-3); Time 2= Follow-up period (i.e., Visits 3 to 5)

$* p<.05 ; * * p<.01$ 
Table 19

Multilevel Models and Piecewise Analyses for CAQ as Associated with Time and Condition, RWE vs. NC

\begin{tabular}{|c|c|c|c|}
\hline & $b$ & $S E$ & $t$ \\
\hline \multicolumn{4}{|l|}{ Total Model Analyses } \\
\hline Intercept & 71.37 & 4.54 & $15.72 * *$ \\
\hline Time & -1.48 & 0.98 & -1.49 \\
\hline Condition & 2.55 & 6.75 & 0.38 \\
\hline Time $\mathrm{x}$ Condition & 2.08 & 1.52 & 1.37 \\
\hline \multicolumn{4}{|l|}{ Contrasts } \\
\hline RWE & -1.48 & 0.98 & -1.49 \\
\hline $\mathrm{NC}$ & 0.61 & 1.15 & 0.53 \\
\hline \multicolumn{4}{|l|}{ Piecewise Analyses } \\
\hline Intercept & 72.59 & 5.21 & $13.93 * *$ \\
\hline Time 1 & -2.34 & 1.87 & -1.25 \\
\hline Time 2 & -1.10 & 1.51 & -0.73 \\
\hline Condition & -1.16 & 7.73 & -0.15 \\
\hline Time 1 x Condition & 4.71 & 2.79 & 1.69 \\
\hline Time $2 \times$ Condition & 0.73 & 2.35 & 0.31 \\
\hline \multicolumn{4}{|l|}{ Time 1 Contrasts } \\
\hline RWE & -2.34 & 1.87 & -1.25 \\
\hline $\mathrm{NC}$ & 2.37 & 2.06 & 1.15 \\
\hline \multicolumn{4}{|l|}{ Time 2 Contrasts } \\
\hline RWE & -1.10 & 1.51 & -0.73 \\
\hline $\mathrm{NC}$ & -0.36 & 1.80 & -0.20 \\
\hline
\end{tabular}

Note. $\mathrm{RWE}=$ Rescripted Written Exposure. $\mathrm{NC}=$ Neutral Control. $\mathrm{CAQ}=$ Cognitive Avoidance Questionnaire (Sexton \& Dugas, 2008).

Under Contrasts, $R W E$ represents within-group changes in the RWE condition, and Neutral represents within-group changes in the control condition.

Under Piecewise Analyses, Time 1= Intervention period, during which writing sessions 1 to 3 occurred (i.e., Visits 1-3); Time 2= Follow-up period (i.e., Visits 3 to 5)

$* p<.05 ; * * p<.01$ 
Table 20

Multilevel Models and Piecewise Analyses for CAQ as Associated with Time and Condition, WE vs. $N C$

\begin{tabular}{|c|c|c|c|}
\hline & $b$ & $S E$ & $t$ \\
\hline \multicolumn{4}{|l|}{ Total Model Analyses } \\
\hline Intercept & 73.82 & 4.61 & $16.02 * *$ \\
\hline Time & -1.81 & 1.01 & $-1.80 * * *$ \\
\hline Condition & 0.24 & 6.79 & 0.03 \\
\hline Time $\mathrm{x}$ Condition & 2.34 & 1.54 & 1.52 \\
\hline \multicolumn{4}{|l|}{ Contrasts } \\
\hline WE & -1.81 & 1.01 & $-1.80 * * *$ \\
\hline $\mathrm{NC}$ & -0.53 & 1.16 & 0.45 \\
\hline \multicolumn{4}{|l|}{ Piecewise Analyses } \\
\hline Intercept & 69.94 & 5.25 & $13.31 * *$ \\
\hline Time 1 & 0.94 & 1.95 & 0.47 \\
\hline Time 2 & -3.22 & 1.65 & $-1.95^{*}$ \\
\hline Condition & 1.51 & 7.71 & 0.20 \\
\hline Time $1 \times$ Condition & 1.42 & 2.88 & 0.49 \\
\hline Time $2 \mathrm{x}$ Condition & 2.93 & 2.55 & 1.15 \\
\hline \multicolumn{4}{|l|}{ Time 1 Contrasts } \\
\hline WE & 0.94 & 1.95 & 0.47 \\
\hline $\mathrm{NC}$ & 2.35 & 2.11 & 1.11 \\
\hline \multicolumn{4}{|l|}{ Time 2 Contrasts } \\
\hline WE & -3.22 & 1.65 & $-1.95 *$ \\
\hline $\mathrm{NC}$ & -0.29 & 1.94 & -0.15 \\
\hline
\end{tabular}

Note. $\mathrm{WE}=$ Worst-Case Scenario Exposure. $\mathrm{NC}=$ Neutral Control. $\mathrm{CAQ}=$ Cognitive Avoidance Questionnaire (Sexton \& Dugas, 2008).

Under Contrasts, WE represents within-group changes in the WE condition, and Neutral represents within-group changes in the control condition.

Under Piecewise Analyses, Time 1= Intervention period, during which writing sessions 1 to 3 occurred (i.e., Visits 1-3); Time 2= Follow-up period (i.e., Visits 3 to 5)

$* p<.05 ; * * p<.01, * * * p=.07$ 


\section{Table 21}

Multilevel Models and Piecewise Analyses for CAQ as Associated with Time and Condition, RWE vs. WE

\begin{tabular}{lccc}
\hline & $b$ & $S E$ & $t$ \\
\hline Total Model Analyses & 71.35 & 3.69 & $19.33^{* *}$ \\
Intercept & -1.47 & 0.99 & -1.47 \\
Time & 2.49 & 5.27 & 0.47 \\
Condition & -0.36 & 1.42 & -0.25 \\
Time x Condition & & & \\
Contrasts & -1.47 & 0.99 & -1.47 \\
RWE & -1.83 & 1.01 & -1.81 \\
WE & & & \\
Piecewise Analyses & 72.59 & 4.51 & $16.08^{* *}$ \\
Intercept & -2.35 & 1.90 & -1.23 \\
Time 1 & -1.11 & 1.56 & -0.71 \\
Time 2 & -2.64 & 6.45 & -0.41 \\
Condition & 3.26 & 2.72 & 1.20 \\
Time 1 x Condition & -2.09 & 2.22 & -0.94 \\
Time 2 x Condition & & & -1.23 \\
Time 1 Contrasts & -2.35 & 1.90 & 0.47 \\
RWE & 0.91 & 1.93 & $-2.02^{*}$ \\
WE & & &
\end{tabular}

Note. $\mathrm{RWE}=$ Rescripted Written Exposure. $\mathrm{WE}=$ Worst-Case Scenario Exposure.

$\mathrm{CAQ}=$ Cognitive Avoidance Questionnaire (Sexton \& Dugas, 2008). Under Contrasts, $R W E$ represents within-group changes in the RWE condition, and WE represents within-group changes in the WE condition.

Under Piecewise Analyses, Time 1= Intervention period, during which writing sessions 1 to 3 occurred (i.e., Visits 1-3); Time 2= Follow-up period (i.e., Visits 3 to 5) $* p<.05 ; * * p<.01$ 
Table 22

Multilevel Models and Piecewise Analyses for NPOQ as Associated with Time and Condition, RWE vs. NC

\begin{tabular}{|c|c|c|c|}
\hline & $b$ & $S E$ & $t$ \\
\hline \multicolumn{4}{|l|}{ Total Model Analyses } \\
\hline Intercept & 42.28 & 2.15 & $19.67 * *$ \\
\hline Time & -0.67 & 0.55 & -1.20 \\
\hline Condition & -3.22 & 3.21 & -1.00 \\
\hline Time $\mathrm{x}$ Condition & 0.65 & 0.86 & 0.76 \\
\hline \multicolumn{4}{|l|}{ Contrasts } \\
\hline RWE & -0.67 & 0.55 & -1.20 \\
\hline $\mathrm{NC}$ & -0.017 & 0.65 & -0.03 \\
\hline \multicolumn{4}{|l|}{ Piecewise Analyses } \\
\hline Intercept & 44.87 & 2.93 & $15.26 * *$ \\
\hline Time 1 & -2.49 & 1.37 & $-1.82 *$ \\
\hline Time 2 & 0.18 & 0.77 & 0.24 \\
\hline Condition & -7.19 & 4.36 & -1.65 \\
\hline Time 1 x Condition & 3.47 & 2.04 & 1.69 \\
\hline Time 2 x Condition & -0.67 & 1.21 & -0.56 \\
\hline \multicolumn{4}{|l|}{ Time 1 Contrasts } \\
\hline RWE & -2.49 & 1.37 & $-1.82 *$ \\
\hline $\mathrm{NC}$ & 0.96 & 1.51 & 0.64 \\
\hline \multicolumn{4}{|l|}{ Time 2 Contrasts } \\
\hline RWE & 0.18 & 0.77 & 0.24 \\
\hline $\mathrm{NC}$ & -0.49 & 0.29 & -0.53 \\
\hline
\end{tabular}

Note. $\mathrm{RWE}=$ Rescripted Written Exposure. $\mathrm{NC}=$ Neutral Control. $\mathrm{NPOQ}=$ Negative Problem Orientation Questionnaire (Gosselin, Pelletier, \& Ladouceur, 2001; Robichaud \& Dugas, 2005). Under Contrasts, $R W E$ represents within-group changes in the RWE condition, and Neutral represents within-group changes in the control condition.

Under Piecewise Analyses, Time 1= Intervention period, during which writing sessions 1 to 3 occurred (i.e., Visits 1-3); Time 2= Follow-up period (i.e., Visits 3 to 5)

$* p<.05 ; * * p<.01$ 
Table 23

Multilevel Models and Piecewise Analyses for NPOQ as Associated with Time and Condition, WE vs. NC

\begin{tabular}{|c|c|c|c|}
\hline & $b$ & $S E$ & $t$ \\
\hline \multicolumn{4}{|l|}{ Total Model Analyses } \\
\hline Intercept & 38.23 & 2.26 & $16.80 * *$ \\
\hline Time & 0.06 & 0.49 & 0.14 \\
\hline Condition & 0.84 & 3.33 & 0.25 \\
\hline Time $\mathrm{x}$ Condition & -0.09 & 0.76 & -0.12 \\
\hline \multicolumn{4}{|l|}{ Contrasts } \\
\hline WE & 0.06 & 0.49 & 0.14 \\
\hline $\mathrm{NC}$ & -0.02 & 0.57 & -0.04 \\
\hline \multicolumn{4}{|l|}{ Piecewise Analyses } \\
\hline Intercept & 38.83 & 2.83 & $13.74 * *$ \\
\hline Time 1 & -0.36 & 1.22 & -0.29 \\
\hline Time 2 & 0.29 & 0.71 & 0.41 \\
\hline Condition & -1.14 & 4.15 & -0.27 \\
\hline Time $1 \times$ Condition & 1.31 & 1.79 & 0.73 \\
\hline Time $2 \mathrm{x}$ Condition & -0.75 & 1.11 & -0.68 \\
\hline \multicolumn{4}{|l|}{ Time 1 Contrasts } \\
\hline WE & -0.36 & 1.22 & -0.29 \\
\hline $\mathrm{NC}$ & 0.95 & 1.31 & 0.72 \\
\hline \multicolumn{4}{|l|}{ Time 2 Contrasts } \\
\hline WE & 0.29 & 0.71 & 0.41 \\
\hline $\mathrm{NC}$ & -0.46 & 0.85 & -0.55 \\
\hline
\end{tabular}

Note. WE = Worst-Case Scenario Exposure. $\mathrm{NC}=$ Neutral Control. $\mathrm{NPOQ}=$ Negative Problem Orientation Questionnaire (Gosselin, Pelletier, \& Ladouceur, 2001; Robichaud \& Dugas, 2005). Under Contrasts, WE represents within-group changes in the WE condition, and Neutral represents within-group changes in the control condition.

Under Piecewise Analyses, Time 1= Intervention period, during which writing sessions 1 to 3 occurred (i.e., Visits 1-3); Time 2= Follow-up period (i.e., Visits 3 to 5)

$* p<.05 ; * * p<.01$ 
Table 24

Multilevel Models and Piecewise Analyses for NPOQ as Associated with Time and Condition, RWE vs. WE

\begin{tabular}{|c|c|c|c|}
\hline & $b$ & $S E$ & $t$ \\
\hline \multicolumn{4}{|l|}{ Total Model Analyses } \\
\hline Intercept & 42.30 & 1.77 & $23.82 * *$ \\
\hline Time & -0.68 & 0.56 & -1.20 \\
\hline Condition & -4.03 & 2.54 & -1.59 \\
\hline Time $\mathrm{x}$ Condition & 0.73 & 0.81 & 0.89 \\
\hline \multicolumn{4}{|l|}{ Contrasts } \\
\hline RWE & -0.68 & 0.56 & -1.20 \\
\hline WE & 0.04 & 0.57 & 0.08 \\
\hline \multicolumn{4}{|l|}{ Piecewise Analyses } \\
\hline Intercept & 44.87 & 2.55 & $17.59 * *$ \\
\hline Time 1 & -2.49 & 1.22 & $-2.04 *$ \\
\hline Time 2 & 0.17 & 0.77 & 0.22 \\
\hline Condition & -6.04 & 3.65 & -1.66 \\
\hline Time 1 x Condition & 2.14 & 1.74 & 1.22 \\
\hline Time $2 \times$ Condition & 0.12 & 1.10 & 0.11 \\
\hline \multicolumn{4}{|l|}{ Time 1 Contrasts } \\
\hline RWE & -2.49 & 1.22 & $-2.04 *$ \\
\hline WE & -0.36 & 1.25 & -0.29 \\
\hline \multicolumn{4}{|l|}{ Time 2 Contrasts } \\
\hline RWE & 0.17 & 0.77 & 0.22 \\
\hline WE & 0.29 & 0.78 & 0.37 \\
\hline
\end{tabular}

Note. $\mathrm{RWE}=$ Rescripted Written Exposure. $\mathrm{WE}=$ Worst-Case Scenario Exposure. $\mathrm{NPOQ}=$ Negative Problem Orientation Questionnaire (Gosselin, Pelletier, \& Ladouceur, 2001; Robichaud \& Dugas, 2005). Under Contrasts, $R W E$ represents within-group changes in the RWE condition, and WE represents withingroup changes in the WE condition.

Under Piecewise Analyses, Time 1= Intervention period, during which writing sessions 1 to 3 occurred (i.e., Visits 1-3); Time $2=$ Follow-up period (i.e., Visits 3 to 5)

$* p<.05 ; * * p<.01$ 
Table 25

Multilevel Models and Piecewise Analyses for ACS as Associated with Time and Condition, RWE vs. $N C$

\begin{tabular}{|c|c|c|c|}
\hline & $b$ & $S E$ & $t$ \\
\hline \multicolumn{4}{|l|}{ Total Model Analyses } \\
\hline Intercept & 168.97 & 6.65 & $25.43 * *$ \\
\hline Time & -2.68 & 1.23 & $-2.18 *$ \\
\hline Condition & -8.16 & 9.87 & -0.83 \\
\hline Time $\mathrm{x}$ Condition & -4.63 & 2.42 & $1.92 * * *$ \\
\hline \multicolumn{4}{|l|}{ Contrasts } \\
\hline RWE & -2.68 & 1.23 & $-2.18^{*}$ \\
\hline $\mathrm{NC}$ & 0.99 & 1.82 & 0.54 \\
\hline \multicolumn{4}{|l|}{ Piecewise Analyses } \\
\hline Intercept & 174.24 & 8.18 & $21.29 * *$ \\
\hline Time 1 & -7.30 & 2.93 & $-2.49 * *$ \\
\hline Time 2 & -1.91 & 2.13 & -0.89 \\
\hline Condition & -11.26 & 12.09 & -0.93 \\
\hline Time 1 x Condition & 6.73 & 4.33 & 1.55 \\
\hline Time $2 \mathrm{x}$ Condition & 4.22 & 3.03 & 1.23 \\
\hline \multicolumn{4}{|l|}{ Time 1 Contrasts } \\
\hline RWE & -7.30 & 2.93 & $-2.49 * *$ \\
\hline $\mathrm{NC}$ & -0.57 & 3.18 & -0.18 \\
\hline \multicolumn{4}{|l|}{ Time 2 Contrasts } \\
\hline RWE & -1.91 & 2.13 & -0.89 \\
\hline $\mathrm{NC}$ & 2.31 & 2.52 & 0.92 \\
\hline
\end{tabular}

Note. $\mathrm{RWE}=$ Rescripted Written Exposure. $\mathrm{NC}=$ Neutral Control. ACS $=$ Affective Control Scale (Williams, Chambless, \& Ahrens, 1997).

Under Contrasts, $R W E$ represents within-group changes in the RWE condition, and Neutral represents within-group changes in the control condition.

Under Piecewise Analyses, Time 1= Intervention period, during which writing sessions 1 to 3 occurred (i.e., Visits 1-3); Time 2= Follow-up period (i.e., Visits 3 to 5)

$* p<.05 ; * * p<.01, * * * p=.08$ 
Table 26

Multilevel Models and Piecewise Analyses for ACS as Associated with Time and Condition, WE vs. $N C$

\begin{tabular}{|c|c|c|c|}
\hline & $b$ & $S E$ & $t$ \\
\hline \multicolumn{4}{|l|}{ Total Model Analyses } \\
\hline Intercept & 166.22 & 5.95 & $27.90 * *$ \\
\hline Time & -3.63 & 1.58 & $-2.29 *$ \\
\hline Condition & -5.35 & 8.77 & -0.61 \\
\hline Time $\mathrm{x}$ Condition & -3.64 & 1.87 & $1.94 *$ \\
\hline \multicolumn{4}{|l|}{ Contrasts } \\
\hline WE & -3.63 & 1.58 & $-2.29 *$ \\
\hline $\mathrm{NC}$ & 0.95 & 1.41 & 0.68 \\
\hline \multicolumn{4}{|l|}{ Piecewise Analyses } \\
\hline Intercept & 171.69 & 7.03 & 24.41 \\
\hline Time 1 & -6.53 & 2.61 & $-2.51 * *$ \\
\hline Time 2 & -0.87 & 1.87 & -0.47 \\
\hline Condition & -8.89 & 10.32 & -0.86 \\
\hline Time $1 \times$ Condition & 6.14 & 3.84 & 1.60 \\
\hline Time $2 \times$ Condition & 2.44 & 2.87 & 0.85 \\
\hline \multicolumn{4}{|l|}{ Time 1 Contrasts } \\
\hline WE & -6.53 & 2.61 & $-2.51 * *$ \\
\hline $\mathrm{NC}$ & -0.39 & 2.81 & -0.14 \\
\hline \multicolumn{4}{|l|}{ Time 2 Contrasts } \\
\hline WE & -0.87 & 1.87 & -0.47 \\
\hline $\mathrm{NC}$ & 1.56 & 2.18 & 0.72 \\
\hline
\end{tabular}

Note. $\mathrm{WE}=$ Worst-Case Scenario Exposure. $\mathrm{NC}=$ Neutral Control. $\mathrm{ACS}=$ Affective Control Scale (Williams, Chambless, \& Ahrens, 1997).

Under Contrasts, WE represents within-group changes in the WE condition, and Neutral represents within-group changes in the control condition.

Under Piecewise Analyses, Time 1= Intervention period, during which writing sessions 1 to 3 occurred (i.e., Visits 1-3); Time 2= Follow-up period (i.e., Visits 3 to 5)

$* p<.05 ; * * p<.01$ 
Table 27

Multilevel Models and Piecewise Analyses for ACS as Associated with Time and Condition, RWE vs. WE

\begin{tabular}{lccc}
\hline & $b$ & $S E$ & $t$ \\
\hline Total Model Analyses & 168.93 & 5.42 & $31.17^{* *}$ \\
Intercept & -3.61 & 1.54 & $-2.35^{*}$ \\
Time & -2.60 & 7.73 & -0.34 \\
Condition & 0.87 & 2.19 & 0.39 \\
Time x Condition & & & \\
Contrasts & -2.73 & 1.56 & $-1.75^{*}$ \\
RWE & -3.61 & 1.54 & $-2.35^{*}$ \\
WE & & & \\
Piecewise Analyses & 174.17 & 7.18 & $24.23^{* *}$ \\
Intercept & -7.27 & 2.95 & $-2.45^{*}$ \\
Time 1 & -1.90 & 2.01 & -0.94 \\
Time 2 & -2.50 & 10.21 & -0.25 \\
Condition & 0.75 & 4.21 & 0.18 \\
Time 1 x Condition & 0.97 & 2.88 & 0.34 \\
Time 2 x Condition & & & $-2.18^{*}$ \\
Time 1 Contrasts & -6.52 & 2.98 & $-2.45^{*}$ \\
RWE & -7.27 & 2.95 & -0.45 \\
WE & & & \\
Time 2 Contrasts & -0.93 & 2.06 & \\
RWE & -1.90 & 501 & \\
WE & & & \\
\hline
\end{tabular}

Note. $\mathrm{RWE}=$ Rescripted Written Exposure. $\mathrm{WE}=$ Worst-Case Scenario Exposure.

ACS = Affective Control Scale (Williams, Chambless, \& Ahrens, 1997).

Under Contrasts, $R W E$ represents within-group changes in the RWE condition, and $W E$ represents within-group changes in the WE condition.

Under Piecewise Analyses, Time 1= Intervention period, during which writing sessions 1 to 3 occurred (i.e., Visits 1-3); Time 2= Follow-up period (i.e., Visits 3 to 5)

$* p<.05 ; * * p<.01$ 
Table 28

Multilevel Models and Piecewise Analyses for ACS Anxiety as Associated with Time and Condition, RWE vs. NC

$b \quad S E$

\begin{tabular}{lccc}
\hline Total Model Analyses & & & \\
Intercept & 56.22 & 2.44 & $22.96^{* *}$ \\
Time & -0.76 & 0.67 & -1.14 \\
Condition & -1.69 & 3.65 & -0.46 \\
Time x Condition & 1.52 & 1.03 & 1.48 \\
Contrasts & & & -1.14 \\
RWE & -0.76 & 0.67 & 0.97 \\
NC & 0.75 & 0.78 &
\end{tabular}

Piecewise Analyses

$\begin{array}{lccc}\text { Intercept } & 55.57 & 3.23 & 17.22 * * \\ \text { Time } 1 & -0.29 & 1.35 & -0.21 \\ \text { Time } 2 & -1.00 & 0.94 & -1.07 \\ \text { Condition } & 0.55 & 4.79 & 0.11 \\ \text { Time 1 x Condition } & -0.07 & 2.02 & -0.03 \\ \text { Time 2 x Condition } & 2.37 & 1.46 & 1.62 \\ \text { me 1 Contrasts } & & & \\ \text { RWE } & -0.29 & 1.35 & -0.21 \\ \text { NC } & -0.36 & 1.49 & -0.25 \\ \text { me 2 Contrasts } & & & -1.07 \\ \text { RWE } & -1.00 & 0.94 & 1.22 \\ \text { NC } & 1.36 & 1.12 & \end{array}$

Note. $\mathrm{RWE}=$ Rescripted Written Exposure. $\mathrm{NC}=$ Neutral Control. ACS Anxiety $=$ Affective Control Scale, Fear of Anxiety Subscale (Williams, Chambless, \& Ahrens, 1997).

Under Contrasts, $R W E$ represents within-group changes in the RWE condition, and Neutral represents within-group changes in the control condition.

Under Piecewise Analyses, Time 1= Intervention period, during which writing sessions 1 to 3 occurred (i.e., Visits 1-3); Time 2= Follow-up period (i.e., Visits 3 to 5)

$* p<.05 ; * * p<.01$ 
Table 29

Multilevel Models and Piecewise Analyses for ACS Anxiety as Associated with Time and Condition, WE vs. NC

\begin{tabular}{|c|c|c|c|}
\hline & $b$ & $S E$ & $t$ \\
\hline \multicolumn{4}{|l|}{ Total Model Analyses } \\
\hline Intercept & 60.91 & 2.14 & $28.44 * *$ \\
\hline Time & -0.49 & 0.50 & -0.98 \\
\hline Condition & -6.39 & 3.16 & $-2.02 *$ \\
\hline Time $\mathrm{x}$ Condition & 1.25 & 0.77 & 1.63 \\
\hline \multicolumn{4}{|l|}{ Contrasts } \\
\hline WE & -0.49 & 0.50 & -0.98 \\
\hline $\mathrm{NC}$ & 0.75 & 0.58 & 1.29 \\
\hline \multicolumn{4}{|l|}{ Piecewise Analyses } \\
\hline Intercept & 62.23 & 2.77 & $22.47 * *$ \\
\hline Time 1 & -1.43 & 1.21 & -1.18 \\
\hline Time 2 & -0.07 & 0.73 & -0.09 \\
\hline Condition & -6.14 & 4.06 & -1.51 \\
\hline Time $1 \times$ Condition & 1.08 & 1.78 & 0.60 \\
\hline Time $2 \times$ Condition & 1.39 & 1.13 & 1.23 \\
\hline \multicolumn{4}{|l|}{ Time 1 Contrasts } \\
\hline WE & -1.43 & 1.21 & -1.18 \\
\hline $\mathrm{NC}$ & -0.35 & 1.31 & -0.27 \\
\hline \multicolumn{4}{|l|}{ Time 2 Contrasts } \\
\hline WE & -0.07 & 0.73 & -0.09 \\
\hline $\mathrm{NC}$ & 1.32 & 0.86 & 1.53 \\
\hline
\end{tabular}

Note. $\mathrm{WE}=$ Worst Case Scenario Exposure. $\mathrm{NC}=$ Neutral Control. ACS Anxiety $=$ Affective Control Scale, Fear of Anxiety Subscale (Williams, Chambless, \& Ahrens, 1997). Under Contrasts, WE represents within-group changes in the WE condition, and Neutral represents within-group changes in the control condition.

Under Piecewise Analyses, Time 1= Intervention period, during which writing sessions 1 to 3 occurred (i.e., Visits 1-3); Time 2= Follow-up period (i.e., Visits 3 to 5)

$* p<.05 ; * * p<.01$ 
Table 30

Multilevel Models and Piecewise Analyses for ACS Anxiety as Associated with Time and Condition, RWE vs. WE

\begin{tabular}{lccc}
\hline & $b$ & $S E$ & $t$ \\
\hline Total Model Analyses & & & \\
Intercept & 56.20 & 2.07 & $27.11^{* *}$ \\
Time & -0.75 & 0.66 & -1.13 \\
Condition & 4.72 & 2.95 & 1.59 \\
Time x Condition & 0.25 & 0.94 & 0.26 \\
Contrasts & & & \\
RWE & -0.49 & 0.67 & -0.74 \\
WE & -0.75 & 0.66 & -1.13 \\
Piecewise Analyses & & & $18.59^{* *}$ \\
Intercept & 55.56 & 2.98 & -0.20 \\
Time 1 & -0.29 & 1.45 & -1.05 \\
Time 2 & -1.02 & 0.97 & 1.56 \\
Condition & 6.67 & 4.27 & -0.55 \\
Time 1 x Condition & -1.14 & 2.08 & 0.70 \\
Time 2 x Condition & 0.97 & 1.37 & -0.05 \\
Time 1 Contrasts & & & -1.05 \\
RWE & -1.43 & 1.48 & -0.20 \\
WE & -0.29 & 1.45 & \\
Time 2 Contrasts & & 0.98 & \\
RWE & -0.05 & 0.97 & \\
WE & -1.02 & & \\
\hline
\end{tabular}

Note. $\mathrm{RWE}=$ Rescripted Written Exposure. $\mathrm{WE}=$ Worst-Case Scenario Exposure. ACS Anxiety= Affective Control Scale, Fear of Anxiety Subscale (Williams, Chambless, \& Ahrens, 1997). Under Contrasts, $R W E$ represents within-group changes in the RWE condition, and $W E$ represents within-group changes in the WE condition.

Under Piecewise Analyses, Time 1= Intervention period, during which writing sessions 1 to 3 occurred (i.e., Visits 1-3); Time 2= Follow-up period (i.e., Visits 3 to 5) $* p<.05 ; * * p<.01$ 
Table 31

Multilevel Models and Piecewise Analyses for ACS Anger as Associated with Time and Condition, RWE vs. NC

\begin{tabular}{lccc}
\hline & $b$ & $S E$ & $t$ \\
\hline Total Model Analyses & & & \\
Intercept & 35.51 & 1.87 & $18.94^{* *}$ \\
Time & -1.23 & 0.44 & $-2.81^{* *}$ \\
Condition & -4.00 & 2.76 & -1.45 \\
Time x Condition & 0.84 & 0.67 & 1.25 \\
Contrasts & & & \\
RWE & -1.23 & 0.44 & $-2.81^{* *}$ \\
NC & -0.39 & 0.51 & -0.79 \\
Piecewise Analyses & & & \\
Intercept & 37.26 & 2.25 & $16.54^{* *}$ \\
Time 1 & -2.47 & 0.79 & $-3.11^{* *}$ \\
Time 2 & -0.59 & 0.64 & -0.92 \\
Condition & -5.42 & 3.29 & -1.65 \\
Time 1 x Condition & 1.84 & 1.16 & 1.57 \\
Time 2 x Condition & 0.22 & 0.99 & 0.22 \\
Time 1 Contrasts & & & -0.92 \\
RWE & -2.47 & 0.79 & $-3.11^{* *}$ \\
NC & -0.63 & 0.84 & -0.75 \\
Time 2 Contrasts & & & \\
RWE & -0.59 & 0.64 & -16 \\
NC & -0.37 & 50.76 & \\
\hline
\end{tabular}

Note. $\mathrm{RWE}=$ Rescripted Written Exposure. $\mathrm{NC}=$ Worst-Case Scenario Exposure. ACS Anger $=$ Affective Control Scale, Fear of Anger Subscale (Williams, Chambless, \& Ahrens, 1997). Under Contrasts, $R W E$ represents within-group changes in the RWE condition, and $N C$ represents within-group changes in the NC condition.

Under Piecewise Analyses, Time 1= Intervention period, during which writing sessions 1 to 3 occurred (i.e., Visits 1-3); Time 2= Follow-up period (i.e., Visits 3 to 5)

$* p<.05 ; * * p<.01$ 
Table 32

Multilevel Models and Piecewise Analyses for ACS Anger as Associated with Time and

Condition, WE vs. NC

\begin{tabular}{|c|c|c|c|}
\hline & $b$ & $S E$ & $t$ \\
\hline \multicolumn{4}{|l|}{ Total Model Analyses } \\
\hline Intercept & 31.67 & 1.73 & $18.31 * *$ \\
\hline Time & -0.77 & 0.44 & -1.75 \\
\hline Condition & -0.16 & 2.58 & -0.06 \\
\hline Time $\mathrm{x}$ Condition & 0.37 & 0.67 & 0.55 \\
\hline \multicolumn{4}{|l|}{ Contrasts } \\
\hline WE & -0.77 & 0.44 & -1.75 \\
\hline $\mathrm{NC}$ & -0.40 & 051 & -0.79 \\
\hline \multicolumn{4}{|l|}{ Piecewise Analyses } \\
\hline Intercept & 31.75 & 2.17 & $14.64 * *$ \\
\hline Time 1 & -0.84 & 0.92 & 0.15 \\
\hline Time 2 & -0.66 & 0.66 & -0.99 \\
\hline Condition & 0.09 & 3.22 & 0.03 \\
\hline Time 1 x Condition & 0.20 & 1.36 & 0.15 \\
\hline Time $2 \times$ Condition & 0.28 & 1.01 & 0.28 \\
\hline \multicolumn{4}{|l|}{ Time 1 Contrasts } \\
\hline WE & -0.84 & 0.92 & 0.15 \\
\hline $\mathrm{NC}$ & -0.63 & 1.01 & -0.63 \\
\hline \multicolumn{4}{|l|}{ Time 2 Contrasts } \\
\hline WE & -0.66 & 0.66 & -0.99 \\
\hline $\mathrm{NC}$ & -0.38 & 0.77 & -0.49 \\
\hline
\end{tabular}

Note. WE = Worst-Case Scenario Exposure. $\mathrm{NC}=$ Neutral Control. ACS Anger = Affective Control Scale, Fear of Anger Subscale (Williams, Chambless, \& Ahrens, 1997).

Under Contrasts, WE represents within-group changes in the WE condition, and $N C$ represents within-group changes in the NC condition.

Under Piecewise Analyses, Time 1= Intervention period, during which writing sessions 1 to 3 occurred (i.e., Visits 1-3); Time 2= Follow-up period (i.e., Visits 3 to 5)

$* p<.05 ; * * p<.01$ 
Table 33

Multilevel Models and Piecewise Analyses for ACS Anger as Associated with Time and

Condition, RWE vs. WE

\begin{tabular}{|c|c|c|c|}
\hline & $b$ & $S E$ & $t$ \\
\hline \multicolumn{4}{|l|}{ Total Model Analyses } \\
\hline Intercept & 35.53 & 1.74 & $20.35^{* *}$ \\
\hline Time & -1.24 & 0.44 & $-2.82 * *$ \\
\hline Condition & -3.82 & 2.44 & -1.56 \\
\hline Time $\mathrm{x}$ Condition & 0.45 & 0.62 & 0.72 \\
\hline \multicolumn{4}{|l|}{ Contrasts } \\
\hline RWE & -1.24 & 0.44 & $-2.82 * *$ \\
\hline WE & -0.79 & 0.44 & -1.79 \\
\hline \multicolumn{4}{|l|}{ Piecewise Analyses } \\
\hline Intercept & 37.24 & 2.25 & $16.49^{* *}$ \\
\hline Time 1 & -2.45 & 3.14 & $-1.74 * *$ \\
\hline Time 2 & -0.65 & 0.60 & -1.07 \\
\hline Condition & -5.48 & 3.14 & -1.75 \\
\hline Time $1 \times$ Condition & 1.61 & 1.27 & 1.26 \\
\hline Time $2 \times$ Condition & -0.005 & 0.86 & -0.006 \\
\hline \multicolumn{4}{|l|}{ Time 1 Contrasts } \\
\hline RWE & -2.45 & 3.14 & $-1.74 * *$ \\
\hline WE & -0.84 & 0.88 & -0.95 \\
\hline \multicolumn{4}{|l|}{ Time 2 Contrasts } \\
\hline RWE & -0.65 & 0.60 & -1.07 \\
\hline WE & -0.65 & 0.62 & -1.05 \\
\hline
\end{tabular}

Note. $\mathrm{RWE}=$ Rescripted Written Exposure. $\mathrm{WE}=$ Worst-Case Scenario Exposure. ACS Anger = Affective Control Scale, Fear of Anger Subscale (Williams, Chambless, \& Ahrens, 1997). Under Contrasts, $R W E$ represents within-group changes in the RWE condition, and $W E$ represents within-group changes in the WE condition.

Under Piecewise Analyses, Time 1= Intervention period, during which writing sessions 1 to 3 occurred (i.e., Visits 1-3); Time 2= Follow-up period (i.e., Visits 3 to 5)

$* p<.05 ; * * p<.01$ 
Table 34

Multilevel Models and Piecewise Analyses for ACS Depressed as Associated with Time and Condition, RWE vs. NC

\begin{tabular}{|c|c|c|c|}
\hline & $b$ & $S E$ & $t$ \\
\hline \multicolumn{4}{|l|}{ Total Model Analyses } \\
\hline Intercept & 32.44 & 2.23 & $14.53 * *$ \\
\hline Time & -0.41 & 0.37 & -1.13 \\
\hline Condition & 2.03 & 3.28 & 0.62 \\
\hline Time $\mathrm{x}$ Condition & 0.75 & 0.56 & 1.34 \\
\hline \multicolumn{4}{|l|}{ Contrasts } \\
\hline RWE & -0.41 & 0.37 & -1.13 \\
\hline $\mathrm{NC}$ & 0.33 & 0.42 & 0.79 \\
\hline \multicolumn{4}{|l|}{ Piecewise Analyses } \\
\hline Intercept & 32.22 & 2.45 & $13.11 * *$ \\
\hline Time 1 & -0.23 & 0.66 & -0.36 \\
\hline Time 2 & -0.50 & 0.55 & -0.90 \\
\hline Condition & 2.37 & 3.60 & 0.66 \\
\hline Time 1 x Condition & 0.49 & 0.98 & 0.50 \\
\hline Time $2 \mathrm{x}$ Condition & 0.92 & 0.86 & 1.06 \\
\hline \multicolumn{4}{|l|}{ Time 1 Contrasts } \\
\hline RWE & -0.23 & 0.66 & -0.36 \\
\hline $\mathrm{NC}$ & 0.24 & 0.71 & 0.35 \\
\hline \multicolumn{4}{|l|}{ Time 2 Contrasts } \\
\hline RWE & -0.50 & 0.55 & -0.90 \\
\hline $\mathrm{NC}$ & 0.41 & 0.66 & 0.62 \\
\hline
\end{tabular}

Note. $\mathrm{RWE}=$ Rescripted Written Exposure. $\mathrm{NC}=$ Worst -Case Scenario Exposure. ACS Depressed = Affective Control Scale, Fear of Depressed Mood Subscale (Williams, Chambless, \& Ahrens, 1997). Under Contrasts, $R W E$ represents within-group changes in the RWE condition, and $N C$ represents within-group changes in the NC condition. Under Piecewise Analyses, Time 1= Intervention period, during which writing sessions 1 to 3 occurred (i.e., Visits 1-3); Time 2= Follow-up period (i.e., Visits 3 to 5) $* p<.05 ; * * p<.01$ 
Table 35

Multilevel Models and Piecewise Analyses for ACS Depressed Mood as Associated with Time and Condition, WE vs. NC

\begin{tabular}{|c|c|c|c|}
\hline & $b$ & $S E$ & $t$ \\
\hline \multicolumn{4}{|l|}{ Total Model Analyses } \\
\hline Intercept & 31.65 & 2.13 & $14.87^{* *}$ \\
\hline Time & -0.32 & 0.42 & -0.77 \\
\hline Condition & 2.81 & 3.17 & 0.88 \\
\hline Time $\mathrm{x}$ Condition & 0.66 & 0.64 & 1.04 \\
\hline \multicolumn{4}{|l|}{ Contrasts } \\
\hline WE & -0.32 & 0.42 & -0.77 \\
\hline $\mathrm{NC}$ & 0.34 & 0.48 & 0.71 \\
\hline \multicolumn{4}{|l|}{ Piecewise Analyses } \\
\hline Intercept & 32.45 & 2.39 & $13.53^{* *}$ \\
\hline Time 1 & -0.89 & 0.73 & -1.22 \\
\hline Time 2 & -0.03 & 0.65 & -0.05 \\
\hline Condition & 2.14 & 3.56 & 0.60 \\
\hline Time 1 x Condition & 1.14 & 1.08 & 1.05 \\
\hline Time $2 \times$ Condition & 0.44 & 0.99 & 0.45 \\
\hline \multicolumn{4}{|l|}{ Time 1 Contrasts } \\
\hline WE & -0.89 & 0.73 & -1.22 \\
\hline $\mathrm{NC}$ & 0.25 & 0.80 & 0.31 \\
\hline \multicolumn{4}{|l|}{ Time 2 Contrasts } \\
\hline WE & -0.03 & 0.65 & -0.05 \\
\hline $\mathrm{NC}$ & 0.41 & 0.75 & 0.55 \\
\hline
\end{tabular}

Note. $\mathrm{WE}=$ Worst -Case Scenario Exposure. $\mathrm{NC}=$ Neutral Control. ACS Depressed $=$ Affective Control Scale, Fear of Depressed Mood Subscale (Williams, Chambless, \& Ahrens, 1997). Under Contrasts, $W E$ represents within-group changes in the WE condition, and $N C$ represents within-group changes in the $\mathrm{NC}$ condition.

Under Piecewise Analyses, Time 1= Intervention period, during which writing sessions 1 to 3 occurred (i.e., Visits 1-3); Time 2= Follow-up period (i.e., Visits 3 to 5)

$* p<.05 ; * * p<.01$ 
Table 36

Multilevel Models and Piecewise Analyses for ACS Depressed as Associated with Time and Condition, RWE vs. WE

\begin{tabular}{|c|c|c|c|}
\hline & $b$ & $S E$ & $t$ \\
\hline \multicolumn{4}{|l|}{ Total Model Analyses } \\
\hline Intercept & 32.43 & 2.12 & $15.26^{* *}$ \\
\hline Time & -0.41 & 0.41 & -0.99 \\
\hline Condition & -0.79 & 2.97 & -0.27 \\
\hline Time $\mathrm{x}$ Condition & 0.09 & 0.58 & 0.16 \\
\hline \multicolumn{4}{|l|}{ Contrasts } \\
\hline RWE & -0.41 & 0.41 & -0.99 \\
\hline WE & -0.32 & 0.41 & -0.76 \\
\hline \multicolumn{4}{|l|}{ Piecewise Analyses } \\
\hline Intercept & 32.25 & 2.52 & $12.79 * *$ \\
\hline Time 1 & -0.26 & 0.82 & -0.32 \\
\hline Time 2 & -0.48 & 0.59 & -0.82 \\
\hline Condition & 0.22 & 3.51 & 0.06 \\
\hline Time $1 \mathrm{x}$ Condition & -0.64 & 1.15 & -0.56 \\
\hline Time $2 \mathrm{x}$ Condition & 0.49 & 0.85 & 0.59 \\
\hline \multicolumn{4}{|l|}{ Time 1 Contrasts } \\
\hline RWE & -0.26 & 0.82 & -0.32 \\
\hline WE & -0.90 & 0.79 & -1.14 \\
\hline \multicolumn{4}{|l|}{ Time 2 Contrasts } \\
\hline RWE & -0.48 & 0.59 & -0.82 \\
\hline WE & 0.009 & 0.60 & 0.02 \\
\hline
\end{tabular}

Note. $\mathrm{RWE}=$ Rescripted Written Exposure. WE = Worst Case Scenario Exposure. ACS Depressed = Affective Control Scale, Fear of Depressed Mood Subscale (Williams, Chambless, \& Ahrens, 1997). Under Contrasts, $R W E$ represents within-group changes in the RWE condition, and $W E$ represents within-group changes in the WE condition.

Under Piecewise Analyses, Time 1= Intervention period, during which writing sessions 1 to 3 occurred (i.e., Visits 1-3); Time 2= Follow-up period (i.e., Visits 3 to 5)

$* p<.05 ; * * p<.01$ 
Table 37

Multilevel Models and Piecewise Analyses for ACS Positive as Associated with Time and Condition, RWE vs. NC

\begin{tabular}{|c|c|c|c|}
\hline & $b$ & $S E$ & $t$ \\
\hline \multicolumn{4}{|l|}{ Total Model Analyses } \\
\hline Intercept & 43.62 & 3.33 & $13.07 * *$ \\
\hline Time & -1.21 & 0.76 & $-1.58 * *$ \\
\hline Condition & -3.95 & 4.91 & -0.81 \\
\hline Time $\mathrm{x}$ Condition & 1.61 & 1.17 & 1.38 \\
\hline \multicolumn{4}{|l|}{ Contrasts } \\
\hline RWE & -1.21 & 0.76 & $-1.58 * *$ \\
\hline $\mathrm{NC}$ & 0.40 & 0.88 & 0.45 \\
\hline \multicolumn{4}{|l|}{ Piecewise Analyses } \\
\hline Intercept & 46.97 & 4.01 & $11.71 * *$ \\
\hline Time 1 & -3.54 & 1.61 & $-2.19 *$ \\
\hline Time 2 & -0.22 & 1.15 & -0.19 \\
\hline Condition & -6.82 & 5.86 & -1.16 \\
\hline Time $1 \mathrm{x}$ Condition & 3.59 & 2.35 & 1.53 \\
\hline Time $2 \times$ Condition & 0.79 & 1.79 & 0.44 \\
\hline \multicolumn{4}{|l|}{ Time 1 Contrasts } \\
\hline RWE & -3.54 & 1.61 & $-2.19 *$ \\
\hline $\mathrm{NC}$ & 0.05 & 1.71 & 0.03 \\
\hline \multicolumn{4}{|l|}{ Time 2 Contrasts } \\
\hline RWE & -0.22 & 1.15 & -0.19 \\
\hline $\mathrm{NC}$ & 0.62 & 0.92 & 0.53 \\
\hline
\end{tabular}

Note. $\mathrm{RWE}=$ Rescripted Written Exposure. $\mathrm{NC}=$ Neutral Control. ACS Positive $=$ Affective Control Scale, Fear of Positive Emotion Subscale (Williams, Chambless, \& Ahrens, 1997). Under Contrasts, $R W E$ represents within-group changes in the RWE condition, and $N C$ represents within-group changes in the NC condition.

Under Piecewise Analyses, Time 1= Intervention period, during which writing sessions 1 to 3 occurred (i.e., Visits 1-3); Time 2= Follow-up period (i.e., Visits 3 to 5)

$* p<.05 ; * * p<.01$ 
Table 38

Multilevel Models and Piecewise Analyses for ACS Positive as Associated with Time and Condition, WE vs. NC

\begin{tabular}{|c|c|c|c|}
\hline & $b$ & $S E$ & $t$ \\
\hline \multicolumn{4}{|l|}{ Total Model Analyses } \\
\hline Intercept & 41.93 & 2.63 & $15.91 * *$ \\
\hline Time & -1.01 & 0.49 & -2.06 \\
\hline Condition & -2.26 & 3.93 & -0.58 \\
\hline Time $\mathrm{x}$ Condition & 1.41 & 0.76 & 1.86 \\
\hline \multicolumn{4}{|l|}{ Contrasts } \\
\hline WE & -1.01 & 0.49 & -2.06 \\
\hline $\mathrm{NC}$ & 0.39 & 0.57 & 0.69 \\
\hline \multicolumn{4}{|l|}{ Piecewise Analyses } \\
\hline Intercept & 45.15 & 3.12 & $14.46^{* *}$ \\
\hline Time 1 & -3.23 & 1.23 & $-2.66 * *$ \\
\hline Time 2 & 0.09 & 0.77 & 0.11 \\
\hline Condition & -4.99 & 4.64 & -1.08 \\
\hline Time 1 x Condition & 3.34 & 1.84 & 1.82 \\
\hline Time $2 \times$ Condition & 0.48 & 1.19 & 0.41 \\
\hline \multicolumn{4}{|l|}{ Time 1 Contrasts } \\
\hline WE & -3.23 & 1.23 & $-2.66 * *$ \\
\hline $\mathrm{NC}$ & 0.05 & 1.36 & 0.04 \\
\hline \multicolumn{4}{|l|}{ Time 2 Contrasts } \\
\hline WE & 0.09 & 0.77 & 0.11 \\
\hline $\mathrm{NC}$ & 0.57 & 0.90 & 0.64 \\
\hline
\end{tabular}

Note. $\mathrm{WE}=$ Worst -Case Scenario Exposure. $\mathrm{NC}=$ Neutral Control. ACS Positive $=$ Affective Control Scale, Fear of Positive Emotion Subscale (Williams, Chambless, \& Ahrens, 1997). Under Contrasts, $W E$ represents within-group changes in the WE condition, and $N C$ represents within-group changes in the NC condition.

Under Piecewise Analyses, Time 1= Intervention period, during which writing sessions 1 to 3 occurred (i.e., Visits 1-3); Time 2= Follow-up period (i.e., Visits 3 to 5)

$* p<.05 ; * * p<.01$ 
Table 39

Multilevel Models and Piecewise Analyses for ACS Positive as Associated with Time and Condition, RWE vs. WE

\begin{tabular}{|c|c|c|c|}
\hline & $b$ & $S E$ & $t$ \\
\hline \multicolumn{4}{|l|}{ Total Model Analyses } \\
\hline Intercept & 43.62 & 3.00 & $14.53 * *$ \\
\hline Time & -1.20 & 0.74 & -1.62 \\
\hline Condition & -1.51 & 4.20 & -0.36 \\
\hline Time $\mathrm{x}$ Condition & 0.08 & 1.05 & 0.07 \\
\hline \multicolumn{4}{|l|}{ Contrasts } \\
\hline RWE & -1.20 & 0.74 & -1.62 \\
\hline WE & -.1 .12 & 0.75 & -1.51 \\
\hline \multicolumn{4}{|l|}{ Piecewise Analyses } \\
\hline Intercept & 47.24 & 3.12 & $15.12 * *$ \\
\hline Time 1 & -3.70 & 1.48 & $-2.50 * *$ \\
\hline Time 2 & -0.08 & 2.15 & -0.04 \\
\hline Condition & -2.20 & 4.33 & -0.51 \\
\hline Time 1 x Condition & 0.53 & 2.05 & 0.26 \\
\hline Time 2 x Condition & -0.12 & 3.06 & -0.04 \\
\hline \multicolumn{4}{|l|}{ Time 1 Contrasts } \\
\hline RWE & -3.70 & 1.48 & $-2.50 * *$ \\
\hline WE & -3.17 & 1.42 & $-2.23 * *$ \\
\hline \multicolumn{4}{|l|}{ Time 2 Contrasts } \\
\hline RWE & -0.08 & 2.15 & -0.04 \\
\hline WE & -0.12 & 3.06 & -0.04 \\
\hline
\end{tabular}

Note. $\mathrm{RWE}=$ Rescripted Written Exposure. $\mathrm{WE}=$ Worst-Case Scenario Exposure. ACS Positive $=$ Affective Control Scale, Fear of Positive Emotion Subscale (Williams, Chambless, \& Ahrens, 1997). Under Contrasts, $R W E$ represents within-group changes in the RWE condition, and $W E$ represents within-group changes in the WE condition.

Under Piecewise Analyses, Time 1= Intervention period, during which writing sessions 1 to 3 occurred (i.e., Visits 1-3); Time 2= Follow-up period (i.e., Visits 3 to 5) $* p<.05 ; * * p<.01$ 
Table 40

Multilevel Models and Piecewise Analyses for Perceived Probability as Associated with Time and Condition, RWE vs. NC

\begin{tabular}{|c|c|c|c|}
\hline & $b$ & $S E$ & $t$ \\
\hline \multicolumn{4}{|l|}{ Total Model Analyses } \\
\hline Intercept & 2.92 & 0.36 & $8.18^{* *}$ \\
\hline Time & 0.05 & 0.10 & 0.51 \\
\hline Condition & 0.49 & 0.53 & 0.93 \\
\hline Time $\mathrm{x}$ Condition & -0.20 & 0.17 & -1.20 \\
\hline \multicolumn{4}{|l|}{ Contrasts } \\
\hline RWE & 0.05 & 0.10 & 0.51 \\
\hline $\mathrm{NC}$ & -0.14 & 0.13 & -1.29 \\
\hline \multicolumn{4}{|l|}{ Piecewise Analyses } \\
\hline Intercept & 2.72 & 0.53 & $5.17 * *$ \\
\hline Time 1 & 0.19 & 0.29 & 0.65 \\
\hline Time 2 & -0.005 & 0.18 & -0.03 \\
\hline Condition & 0.44 & 0.78 & 0.56 \\
\hline Time $1 \mathrm{x}$ Condition & -0.15 & 0.44 & -0.35 \\
\hline Time $2 \times$ Condition & -0.22 & 0.28 & -0.79 \\
\hline \multicolumn{4}{|l|}{ Time 1 Contrasts } \\
\hline RWE & -0.15 & 0.44 & -0.35 \\
\hline $\mathrm{NC}$ & 0.19 & 0.29 & 0.65 \\
\hline \multicolumn{4}{|l|}{ Time 2 Contrasts } \\
\hline RWE & -0.23 & 2.18 & -1.06 \\
\hline $\mathrm{NC}$ & -0.005 & 0.18 & -0.03 \\
\hline
\end{tabular}

Note. $\mathrm{RWE}=$ Rescripted Written Exposure. $\mathrm{NC}=$ Neutral Control. Perceived Probability $=$ Perceived Probability Questions (Berenbaum et al., 2007; Butler \& Mathews, 1983). Under Contrasts, $R W E$ represents within-group changes in the RWE condition, and Neutral represents within-group changes in the control condition.

Under Piecewise Analyses, Time 1= Intervention period, during which writing sessions 1 to 3 occurred (i.e., Visits 1-3); Time 2= Follow-up period (i.e., Visits 3 to 5)

$* p<.05 ; * * p<.01$ 
Table 41

Multilevel Models and Piecewise Analyses for Perceived Probability as Associated with Time and Condition, WE vs. NC

\begin{tabular}{|c|c|c|c|}
\hline & $b$ & $S E$ & $t$ \\
\hline \multicolumn{4}{|l|}{ Total Model Analyses } \\
\hline Intercept & 3.01 & 0.34 & $8.79 * *$ \\
\hline Time & -0.14 & 0.09 & $-1.45 *$ \\
\hline Condition & 0.39 & 0.50 & 0.44 \\
\hline Time $\mathrm{x}$ Condition & -0.26 & 0.12 & $-2.13^{*}$ \\
\hline \multicolumn{4}{|l|}{ Contrasts } \\
\hline WE & -0.14 & 0.09 & $-1.45^{*}$ \\
\hline $\mathrm{NC}$ & 0.12 & 0.08 & 0.78 \\
\hline \multicolumn{4}{|l|}{ Piecewise Analyses } \\
\hline Intercept & 2.84 & 0.46 & $6.17 * *$ \\
\hline Time 1 & 0.24 & 0.22 & 1.11 \\
\hline Time 2 & 0.07 & 0.14 & 0.52 \\
\hline Condition & 0.30 & 0.67 & 0.45 \\
\hline Time $1 \mathrm{x}$ Condition & -0.19 & 0.32 & -0.61 \\
\hline Time 2 x Condition & -0.32 & 0.21 & -1.51 \\
\hline \multicolumn{4}{|l|}{ Time 1 Contrasts } \\
\hline WE & 0.24 & 0.22 & 1.11 \\
\hline $\mathrm{NC}$ & 0.04 & 0.23 & 0.20 \\
\hline \multicolumn{4}{|l|}{ Time 2 Contrasts } \\
\hline WE & 0.07 & 0.14 & 0.52 \\
\hline $\mathrm{NC}$ & -0.24 & 0.16 & -1.55 \\
\hline
\end{tabular}

Note. $\mathrm{WE}=$ Worst-Case Scenario Exposure $. \mathrm{NC}=$ Neutral Control . Perceived Probability = Perceived Probability Questions (Berenbaum et al., 2007; Butler \& Mathews, 1983). Under Contrasts, WE represents within-group changes in the WE condition, and Neutral represents within-group changes in the control condition.

Under Piecewise Analyses, Time 1= Intervention period, during which writing sessions 1 to 3 occurred (i.e., Visits 1-3); Time 2= Follow-up period (i.e., Visits 3 to 5)

$* p<.05 ; * * p<.01$ 
Table 42

Multilevel Models and Piecewise Analyses for Perceived Probability as Associated with Time and Condition, RWE vs. WE

\begin{tabular}{|c|c|c|c|}
\hline & $b$ & $S E$ & $t$ \\
\hline \multicolumn{4}{|l|}{ Total Model Analyses } \\
\hline Intercept & 2.92 & 0.33 & $8.90 * *$ \\
\hline Time & 0.05 & 0.10 & 0.54 \\
\hline Condition & 0.09 & 0.47 & 0.21 \\
\hline Time $\mathrm{x}$ Condition & 0.07 & 0.15 & 0.46 \\
\hline \multicolumn{4}{|l|}{ Contrasts } \\
\hline RWE & 0.05 & 0.10 & 0.54 \\
\hline WE & 0.12 & 0.11 & 1.16 \\
\hline \multicolumn{4}{|l|}{ Piecewise Analyses } \\
\hline Intercept & 2.72 & 0.50 & $5.41 * *$ \\
\hline Time 1 & 0.19 & 0.29 & 0.67 \\
\hline Time 2 & -0.006 & 0.17 & -0.03 \\
\hline Condition & 0.11 & 0.72 & 0.15 \\
\hline Time $1 \mathrm{x}$ Condition & 0.06 & 0.42 & 0.15 \\
\hline Time 2 x Condition & 0.07 & 0.24 & 0.28 \\
\hline \multicolumn{4}{|l|}{ Time 1 Contrasts } \\
\hline RWE & 0.19 & 0.29 & 0.67 \\
\hline WE & 0.25 & 0.29 & 0.87 \\
\hline \multicolumn{4}{|l|}{ Time 2 Contrasts } \\
\hline RWE & -0.006 & 0.17 & -0.03 \\
\hline WE & 0.06 & 0.18 & 0.36 \\
\hline
\end{tabular}

Note. $\mathrm{RWE}=$ Rescripted Written Exposure. $\mathrm{WE}=$ Worst-Case Scenario Exposure. Perceived Probability = Perceived Probability Questions (Berenbaum et al., 2007; Butler \& Mathews, 1983). Under Contrasts, $R W E$ represents within-group changes in the RWE condition, and WE represents within-group changes in the WE condition. Under Piecewise Analyses, Time 1= Intervention period, during which writing sessions 1 to 3 occurred (i.e., Visits 1-3); Time 2= Follow-up period (i.e., Visits 3 to 5) $* p<.05 ; * * p<.01$ 
Table 43

Multilevel Models and Piecewise Analyses for Perceived Cost as Associated with Time and Condition, RWE vs. NC

\begin{tabular}{|c|c|c|c|}
\hline & $b$ & $S E$ & $t$ \\
\hline \multicolumn{4}{|l|}{ Total Model Analyses } \\
\hline Intercept & 5.20 & 0.24 & $22.03 * *$ \\
\hline Time & -0.22 & 0.10 & $-2.10^{*}$ \\
\hline Condition & 0.25 & 0.35 & 0.72 \\
\hline Time $\mathrm{x}$ Condition & 0.09 & 0.16 & 0.54 \\
\hline \multicolumn{4}{|l|}{ Contrasts } \\
\hline RWE & -0.22 & 0.10 & $-2.1 *$ \\
\hline $\mathrm{NC}$ & -0.13 & 0.13 & -1.10 \\
\hline \multicolumn{4}{|l|}{ Piecewise Analyses } \\
\hline Intercept & 5.56 & 0.39 & $14.29 * *$ \\
\hline Time 1 & -0.47 & 0.23 & $-2.09 *$ \\
\hline Time 2 & -0.09 & 0.18 & -0.53 \\
\hline Condition & -0.55 & 0.58 & -0.96 \\
\hline Time 1 x Condition & 0.67 & 0.34 & $1.98 *$ \\
\hline Time 2 x Condition & -0.19 & 0.28 & -0.69 \\
\hline \multicolumn{4}{|l|}{ Time 1 Contrasts } \\
\hline RWE & -0.47 & 0.23 & $-2.09 *$ \\
\hline $\mathrm{NC}$ & 0.19 & 0.25 & 0.78 \\
\hline \multicolumn{4}{|l|}{ Time 2 Contrasts } \\
\hline RWE & -0.29 & 0.22 & -1.34 \\
\hline $\mathrm{NC}$ & 0.67 & 0.34 & 1.98 \\
\hline
\end{tabular}

Note. RWE $=$ Rescripted Written Exposure. $\mathrm{NC}=$ Neutral Control. Perceived Cost $=$ Perceived Cost Questions (Berenbaum et al., 2007; Butler \& Mathews, 1983).

Under Contrasts, $R W E$ represents within-group changes in the RWE condition, and Neutral represents within-group changes in the control condition.

Under Piecewise Analyses, Time 1= Intervention period, during which writing sessions 1 to 3 occurred (i.e., Visits 1-3); Time 2= Follow-up period (i.e., Visits 3 to 5)

$* p<.05 ; * * p<.01$ 
Table 44

Multilevel Models and Piecewise Analyses for Perceived Cost as Associated with Time and

Condition, WE vs. NC

\begin{tabular}{|c|c|c|c|}
\hline & $b$ & $S E$ & $t$ \\
\hline \multicolumn{4}{|l|}{ Total Model Analyses } \\
\hline Intercept & 4.95 & 0.23 & $21.16^{* *}$ \\
\hline Time & 0.008 & 0.10 & 0.09 \\
\hline Condition & 0.52 & 0.35 & 1.50 \\
\hline Time $\mathrm{x}$ Condition & -0.14 & 0.15 & -0.95 \\
\hline \multicolumn{4}{|l|}{ Contrasts } \\
\hline WE & 0.008 & 0.10 & 0.09 \\
\hline $\mathrm{NC}$ & -0.14 & 0.11 & -1.12 \\
\hline \multicolumn{4}{|l|}{ Piecewise Analyses } \\
\hline Intercept & 5.09 & 0.41 & 12.48 \\
\hline Time $1^{\mathrm{a}}$ & -0.09 & 0.24 & -0.16 \\
\hline Time $2^{\mathrm{b}}$ & 0.06 & 0.14 & 0.44 \\
\hline Condition & -0.09 & 0.59 & -0.16 \\
\hline Time 1 x Condition & 0.29 & 0.35 & 0.86 \\
\hline Time 2 x Condition & -0.38 & 0.22 & -1.73 \\
\hline \multicolumn{4}{|l|}{ Time 1 Contrasts } \\
\hline WE & -0.09 & 0.24 & -0.16 \\
\hline $\mathrm{NC}$ & 0.20 & 0.26 & 0.78 \\
\hline \multicolumn{4}{|l|}{ Time 2 Contrasts } \\
\hline WE & 0.06 & 0.14 & 0.44 \\
\hline $\mathrm{NC}$ & -0.31 & 0.16 & -1.91 \\
\hline
\end{tabular}

Note. $\mathrm{WE}=$ Worst -Case Scenario Exposure. $\mathrm{NC}=$ Neutral Control. Perceived Cost $=$ Perceived Cost Questions (Berenbaum et al., 2007; Butler \& Mathews, 1983).

Under Contrasts, WE represents within-group changes in the WE condition, and Neutral represents within-group changes in the control condition.

Under Piecewise Analyses, Time 1= Intervention period, during which writing sessions 1 to 3 occurred (i.e., Visits 1-3); Time 2= Follow-up period (i.e., Visits 3 to 5)

$* p<.05 ; * * p<.01$ 
Table 45

Multilevel Models and Piecewise Analyses for Perceived Cost as Associated with Time and Condition, RWE vs. WE

\begin{tabular}{|c|c|c|c|}
\hline & $b$ & $S E$ & $t$ \\
\hline \multicolumn{4}{|l|}{ Total Model Analyses } \\
\hline Intercept & 5.21 & 0.22 & $23.37 * *$ \\
\hline Time & -0.22 & 0.10 & $-2.12^{*}$ \\
\hline Condition & -0.25 & 0.32 & -0.78 \\
\hline Time $\mathrm{x}$ Condition & 0.22 & 0.14 & 1.56 \\
\hline \multicolumn{4}{|l|}{ Contrasts } \\
\hline RWE & -0.22 & 0.10 & $-2.12 *$ \\
\hline WE & 0.005 & 0.10 & 0.05 \\
\hline \multicolumn{4}{|l|}{ Piecewise Analyses } \\
\hline Intercept & 5.55 & 0.38 & $14.43 * *$ \\
\hline Time 1 & -0.47 & 0.23 & $-2.1 *$ \\
\hline Time 2 & -0.09 & 0.17 & -0.57 \\
\hline Condition & -0.46 & 0.55 & -0.84 \\
\hline Time 1 x Condition & 0.38 & 0.32 & 1.17 \\
\hline Time $2 \mathrm{x}$ Condition & 0.15 & 0.24 & 0.59 \\
\hline \multicolumn{4}{|l|}{ Time 1 Contrasts } \\
\hline RWE & -0.47 & 0.23 & $-2.10^{*}$ \\
\hline WE & -0.09 & 0.23 & -0.39 \\
\hline \multicolumn{4}{|l|}{ Time 2 Contrasts } \\
\hline RWE & -0.09 & 0.17 & -0.57 \\
\hline WE & 0.04 & 0.18 & 0.27 \\
\hline
\end{tabular}

Note. $\mathrm{RWE}=$ Rescripted Written Exposure. WE = Worst-Case Scenario Exposure.

Perceived Cost $=$ Perceived Cost Questions (Berenbaum et al., 2007; Butler \& Mathews, 1983). Under Contrasts, $R W E$ represents within-group changes in the RWE condition, and $W E$ represents within-group changes in the WE condition.

Under Piecewise Analyses, Time 1= Intervention period, during which writing sessions 1 to 3 occurred (i.e., Visits 1-3); Time 2= Follow-up period (i.e., Visits 3 to 5)

$* p<.05 ; * * p<.01$ 
Table 46

Multilevel Models and Piecewise Analyses for Perceived Coping as Associated with Time and Condition, RWE vs. NC

\begin{tabular}{|c|c|c|c|}
\hline & $b$ & $S E$ & $t$ \\
\hline \multicolumn{4}{|l|}{ Total Model Analyses } \\
\hline Intercept & 2.51 & 0.35 & $7.24 * *$ \\
\hline Time & 0.07 & 0.15 & 0.49 \\
\hline Condition & -0.06 & 0.52 & -0.11 \\
\hline Time $\mathrm{x}$ Condition & -0.009 & 0.23 & -0.04 \\
\hline \multicolumn{4}{|l|}{ Contrasts } \\
\hline RWE & 0.07 & 0.15 & 0.49 \\
\hline $\mathrm{NC}$ & 0.06 & 0.18 & 0.36 \\
\hline \multicolumn{4}{|l|}{ Piecewise Analyses } \\
\hline Intercept & 1.08 & 0.59 & $1.81 * *$ \\
\hline Time 1 & 1.08 & 0.34 & $3.18 * *$ \\
\hline Time 2 & -0.37 & 0.21 & -1.81 \\
\hline Condition & 1.83 & 0.88 & 2.06 \\
\hline Time 1 x Condition & 1.34 & 0.51 & $2.63 * *$ \\
\hline Time 2 x Condition & 0.61 & 0.32 & 1.87 \\
\hline \multicolumn{4}{|l|}{ Time 1 Contrasts } \\
\hline RWE & 1.08 & 0.34 & $3.18 * *$ \\
\hline $\mathrm{NC}$ & -0.25 & 0.38 & -0.67 \\
\hline \multicolumn{4}{|l|}{ Time 2 Contrasts } \\
\hline RWE & -0.37 & 0.21 & -1.81 \\
\hline $\mathrm{NC}$ & 0.22 & 0.25 & 0.93 \\
\hline
\end{tabular}

Note. $\mathrm{RWE}=$ Rescripted Written Exposure $. \mathrm{NC}=$ Neutral Control. Perceived Coping = Perceived Coping Questions (Berenbaum et al., 2007; Butler \& Mathews, 1983).

Under Contrasts, $R W E$ represents within-group changes in the RWE condition, and Neutral represents within-group changes in the control condition.

Under Piecewise Analyses, Time 1= Intervention period, during which writing sessions 1 to 3 occurred (i.e., Visits 1-3); Time 2= Follow-up period (i.e., Visits 3 to 5)

$* p<.05 ; * * p<.01$ 
Table 47

Multilevel Models and Piecewise Analyses for Perceived Coping as Associated with Time and Condition, WE vs. NC

\begin{tabular}{|c|c|c|c|}
\hline & $b$ & $S E$ & $t$ \\
\hline \multicolumn{4}{|l|}{ Total Model Analyses } \\
\hline Intercept & 2.64 & 0.38 & $6.86^{* *}$ \\
\hline Time & 0.05 & 0.12 & 0.37 \\
\hline Condition & -0.18 & 0.57 & 0.32 \\
\hline Time $\mathrm{x}$ Condition & 0.018 & 0.18 & 0.09 \\
\hline \multicolumn{4}{|l|}{ Contrasts } \\
\hline WE & 0.05 & 0.12 & 0.37 \\
\hline $\mathrm{NC}$ & 0.04 & 0.12 & 0.45 \\
\hline \multicolumn{4}{|l|}{ Piecewise Analyses } \\
\hline Intercept & 2.21 & 0.58 & $3.77 * *$ \\
\hline Time 1 & 0.35 & 0.32 & 1.08 \\
\hline Time 2 & -0.09 & 0.20 & -0.49 \\
\hline Condition & 0.69 & 0.86 & 0.42 \\
\hline Time $1 \times$ Condition & -0.60 & 0.48 & -1.25 \\
\hline Time $2 \times$ Condition & 0.33 & 0.31 & 1.07 \\
\hline \multicolumn{4}{|l|}{ Time 1 Contrasts } \\
\hline WE & 0.35 & 0.32 & 1.08 \\
\hline $\mathrm{NC}$ & -0.25 & 0.35 & -0.70 \\
\hline \multicolumn{4}{|l|}{ Time 2 Contrasts } \\
\hline WE & -0.09 & 0.20 & -0.49 \\
\hline $\mathrm{NC}$ & 0.23 & 0.23 & 0.99 \\
\hline
\end{tabular}

Note. $\mathrm{WE}=$ Worst-Case Scenario Exposure. $\mathrm{NC}=$ Neutral Control. Perceived Coping = Perceived Coping Questions (Berenbaum et al., 2007; Butler \& Mathews, 1983). Under Contrasts, WE represents within-group changes in the WE condition, and Neutral represents within-group changes in the control condition.

Under Piecewise Analyses, Time 1= Intervention period, during which writing sessions 1 to 3 occurred (i.e., Visits 1-3); Time 2= Follow-up period (i.e., Visits 3 to 5) $* p<.05 ; * * p<.01$ 
Table 48

Multilevel Models and Piecewise Analyses for Perceived Coping as Associated with Time and Condition, RWE vs. WE

\begin{tabular}{|c|c|c|c|}
\hline & $b$ & $S E$ & $t$ \\
\hline \multicolumn{4}{|l|}{ Total Model Analyses } \\
\hline Intercept & 2.51 & 0.33 & $7.26^{* *}$ \\
\hline Time & 0.07 & 0.14 & 0.53 \\
\hline Condition & 0.12 & 0.47 & 0.26 \\
\hline Time $\mathrm{x}$ Condition & -0.02 & 0.20 & -0.12 \\
\hline \multicolumn{4}{|l|}{ Contrasts } \\
\hline RWE & 0.07 & 0.14 & 0.53 \\
\hline WE & 0.05 & 0.15 & 0.33 \\
\hline \multicolumn{4}{|l|}{ Piecewise Analyses } \\
\hline Intercept & 1.08 & 0.59 & $1.82 * *$ \\
\hline Time 1 & 1.09 & 0.34 & $3.18^{* *}$ \\
\hline Time 2 & -0.37 & 0.19 & $-1.97 *$ \\
\hline Condition & 1.13 & 0.84 & 1.34 \\
\hline Time 1 x Condition & -0.73 & 0.49 & -1.49 \\
\hline Time $2 \times$ Condition & 0.26 & 0.27 & 0.97 \\
\hline \multicolumn{4}{|l|}{ Time 1 Contrasts } \\
\hline RWE & 1.09 & 0.34 & $3.18^{* *}$ \\
\hline WE & 0.35 & 0.35 & 1.01 \\
\hline \multicolumn{4}{|l|}{ Time 2 Contrasts } \\
\hline RWE & -0.11 & 0.19 & -0.55 \\
\hline WE & -0.37 & 0.19 & $-1.97 *$ \\
\hline
\end{tabular}

Note. $\mathrm{RWE}=$ Rescripted Written Exposure. $\mathrm{WE}=$ Worst-Case Scenario Exposure. Perceived Coping = Perceived Coping Questions (Berenbaum et al., 2007; Butler \& Mathews, 1983). Under Contrasts, $R W E$ represents within-group changes in the RWE condition, and $W E$ represents within-group changes in the WE condition.

Under Piecewise Analyses, Time 1= Intervention period, during which writing sessions 1 to 3 occurred (i.e., Visits 1-3); Time 2= Follow-up period (i.e., Visits 3 to 5)

$* p<.05 ; * * p<.01$ 


\section{Appendix A}

\section{General Instructions}

This study is a very important project looking at writing. Over the next three days, you will be asked to write about a topic for 30 minutes each day. Your instructions for writing will be located on the back of the front page of the booklet to be given to you at each writing session. You will complete your writing alone in a private room. After you finish reading your writing instructions for the day, I will leave the room and close the door. The closing of the door will be your signal to start writing. Fifteen minutes into the writing, I will knock on your door and ask you to complete a few measures. When the writing session is over, I will knock on your door to let you know and you are to stop writing. The only rule we have about writing is that you write continuously for the entire time. If you run out of things to say, just repeat what you have already written. In your writing, don't worry about grammar, spelling, or sentence structure. Just write.

Your writing is completely confidential. You are identified by an ID number, which is written on the front of the booklet. Please do not write your name or any other identifying information anywhere on your writing sample. 


\section{Appendix B}

\section{WE Instructions}

Day 1: Today, please write a story about your worst fear coming true. In your writing, I want you to really let go and explore your very deepest emotions and thoughts.

- Start by describing the circumstances that lead to the situation, then describe what happens during the situation, and finally the consequences of the situation. In other words, tell a story about what happens, how it turns out, and how it makes you think and feel.

- Do not worry about grammar, spelling or sentence structure.

$\circ$ Write in first person, present tense, as if the situation is really happening in the here-andnow.

- Include your physical sensations. For example, describe how your body is reacting and what you feel, touch, taste and smell.

You may feel anxious when writing thoughts, feelings and sensations about your worst fear coming true - this is normal.

Day 2: Today please continue to write about the same situation that you wrote about yesterday your worst fear coming true.

- Remember to write in first person and in present tense, as if the situation is happening in the here-and-now.

- You may change your thoughts, feelings or description but make sure you are writing about the same worst fear coming true.

○ Today we really want you to explore your very deepest thoughts and emotions.

Day 3: Today is the last writing session. Please continue to write about the same situation that you wrote about yesterday - your worst fear coming true.

$\circ$ Remember to write in first person and in present tense, as if the situation is happening in the here-and-now.

○ You may change your thoughts, feelings or description, but make sure you are writing about the same worst fear coming true.

$\circ$ We again want you to explore your deepest thoughts and feelings about your worst fear coming true. 


\title{
RWE Instructions
}

\author{
Appendix C
}

Day 1: Today, please write a story about your worst fear coming true. In your writing, I want you to really let go and explore your very deepest emotions and thoughts.

- Start by describing the circumstances that lead to the situation, then describe what happens during the situation, and finally the consequences of the situation. In other words, tell a story about what happens, how it turns out, and how it makes you think and feel.

- Do not worry about grammar, spelling or sentence structure.

$\circ$ Write in first person, present tense, as if the situation is really happening in the here-andnow.

- Include your physical sensations. For example, describe how your body is reacting and what you feel, touch, taste and smell.

You may feel anxious when writing thoughts, feelings and sensations about your worst fear coming true- - this is normal.

Day 2: Today please continue to write about the same situation that you wrote about yesterday your worst fear coming true.

Day 2: Today please continue to write about the same situation that you wrote about yesterday your worst fear coming true.

- Start by describing the circumstances that lead to the situation, then describe what happens during the situation, and the consequences of the situation

- But today, please also write about how you move forward to improve or adapt to the situation (for example, resolve a conflict with another person, change something negative or aversive in your environment, pursue a new opportunity). What is the first step you take to improve the situation? And the next?

- Please also write about how you could change your attitude about the situation

- Again, write your whole story in first person, present tense, as if you are responding to the situation in the here-and-now.

○ Include your thoughts, feelings, and descriptions of the events.

- Today we really want you to explore your very deepest thoughts, emotions and descriptions of the events.

Day 3: Today is the last writing session. Your worst-fear coming true is happening. Please write again about how you move forward to improve or adapt to the situation.

○ You may change your thoughts, feelings or description but make sure you write about how you move forward to improve or adapt to the situation and how you could change your attitude about the situation.

- What is the first step you can take? And the next?

- Remember to write in first person, present tense, as if you are responding to the situation in the here-and-now.

- Today we really want you to explore your very deepest thoughts, emotions and descriptions of the events. 


\section{Appendix D}

\section{NC Instructions}

Day 1: What you are to write about over the next 3 days is how you use your time. Each day you will be given a writing assignment on the way you spend your time.

O In today's writing, describe what you would do with your day if you found out you had today off from school or work.

- In your writing, be as objective as possible.

- Do not write about your emotions, thoughts, opinions, or reactions. Rather, try to be completely factual.

- Feel free to be as detailed as possible.

Day 2: Today, please describe what you would do with your day if you found out you had today off from school or work.

○ Again, be as objective as possible, with no description of emotions, thoughts, opinions, or reactions.

Day 3: You have written now for two days and today is the last writing session. In your writing today, describe what you would do with your day if you found out you had today off from school or work.

○ Again, be as objective as possible, with no description of emotions, thoughts, opinions, or reactions. 


\section{Appendix E-Consent Form}

Information and Consent Form

Worry and Writing Study

You are being asked to participate in a research study. Before you give your consent to be a volunteer, it is important that you read the following information and ask as many questions as necessary to be sure you understand what you will be asked to do.

\section{Investigators:}

Melina Ovanessian, M.A. Student, Department of Psychology, Ryerson University

Naomi Koerner, Ph.D., Associate Professor, Department of Psychology, Ryerson University

Martin M. Antony, Ph.D., Professor, Department of Psychology, Ryerson University

Purpose of the Study: The purpose of this study is to assess the relationship between worry and writing. Although research has shown that writing leads to positive health and psychological outcomes, the relationship between writing and worry has not been thoroughly investigated. This research is being conducted in partial fulfilment of Melina Ovanessian's Master of Arts degree in Psychology and is being supervised by Drs. Koerner and Antony.

Description of the Study: The experiment will involve five visits to the Psychology Research and Training Centre at Ryerson University, located at 105 Bond Street. The total time commitment for the study is 4.5 hours.

Visit 1 (duration $=\mathbf{2}$ hours). After providing written informed consent, you will be asked to participate in a 30 to 45 minute interview with Melina Ovanessian in which you will be asked questions about current emotional and psychological experiences (for example, worry and anxiety). Please note that the interview will be audiorecorded to ensure accurate interpretation of your responses. The audiorecording will not have your name on it and it will be listened to only by research assistants for the purpose of this research. The audiorecording will be stored in a password protected area on the Ryerson server and will be encrypted (that is, encoded a certain way so no one can listen to the audiorecording without a password).

If you are found to be eligible for continued participation in the study following the interview, you will then complete a set of questionnaires about your thoughts, emotions, and behaviour. Next, you will be randomly assigned (like flipping a coin) to one of three types of writing tasks, each of which involves writing about a topic according to a specific set of instructions, for 3 days in a row, starting at Visit 1. You will then go onto your first writing session, which will last approximately 30 minutes. You will earn $\$ 20$ at the end of Visit 1, regardless of whether or not you are eligible for continued participation in the study after the interview.

Visit 2 (the day after Visit 1; duration $=\mathbf{3 0}$ minutes): You will be asked to return to the lab to engage in your second 30-minute writing session. You will earn $\$ 5$ at the end of Visit 2. 
Visit 3 (the day after Visit 2; duration = 1 hour). You will be asked to return to the lab again to engage in your third 30-minute writing session. After this, you will be asked to complete the same questionnaires as in Visit 1. You will earn $\$ 10$ at the end of the Visit 3.

Visit 4 (1 week after Visit 3; duration = 30 minutes). You will be asked to return to the lab to complete the questionnaires that you completed at Visits 1 and 3. You will earn $\$ 10$ for this visit.

Visit 5 (1 month after Visit 3; duration = 30 minutes): You will be asked to return to the lab to complete the questionnaires that you completed at Visits 1, 3 and 4. You will earn $\$ 10$ for this visit.

Potential Risks or Discomforts: There is minimal risk involved if you agree to take part in this study. You understand that you may experience some negative emotions when completing the questionnaires and writing tasks. You have the right to refuse or discontinue participation at any time. If you decide to stop participating, you will still be entitled to compensation for the phases of the research that you started.

Potential Benefits of the Study To You or Others: I cannot guarantee that you will receive any benefits from participating in this study. You may derive benefit from the self-assessment as it may increase your awareness of your emotions and behaviours. You may also develop a better understanding of research methodology and will be providing researchers with valuable insight.

Confidentiality: Everything you disclose in this study will remain completely confidential; however, as part of this study, we are obligated to inform everyone that there are five cases in which we might need to break confidentiality:

(1) If you intend to harm yourself;

(2) if you intend on harming someone else;

(3) If there is reasonable suspicion that a child up to the age of 16 years is at risk of neglect or abuse, we are required by law to report this to the Children's Aid Society right away;

(4) if our files are subpoenaed by the courts (records can be opened by a specific court order, but it is highly unlikely that this would ever happen); and

(5) if a regulated health professional has engaged in inappropriate sexual behaviour toward you and you provide us with the name of this individual, we are obligated to report them to their regulatory body. 
This informed consent agreement and all data with information that identifies you will be stored in locked file cabinets at the Psychology Research and Training Centre at Ryerson University. An ID number as opposed to your name will be entered on your phone screen, interview, questionnaires, your writing booklets, and in all computer files that will contain the data you generate during the study. The data you generate while participating in this study will be kept in a locked file cabinet and on a secure server, separate from this consent agreement and any data that identifies you.

Your phone screen, interview, questionnaires and writing booklets will be shredded 10 years after the final findings have been published. Any data stored in electronic files (for example, questionnaire scores, typed versions of the writing you did during the writing sessions, audiorecordings of the interview) will be kept indefinitely. Your confidentiality will be protected to the full extent allowed by law. Group findings will be reported in publications and presentations arising from this research. Quotes from your writing may be included in publications and presentations as illustrations/examples of what participants wrote about in the study; but please note that your name and any other identifying information will not appear with the quotes.

Compensation for Participation: You will earn up to $\$ 55$ depending upon the number of visits you make to the study. You are asked to arrange to transport yourself to the Psychology Research and Training Centre at Ryerson University. You will not be paid for the telephone screen that you took part in to determine eligibility.

Voluntary Nature of Participation: Participation in this study is voluntary. Your choice of whether or not to participate will not influence your future relations with Ryerson University. If you decide to participate, you are free to withdraw your consent and to stop your participation at any time without penalty or loss of benefits to which you are allowed. If you decide to withdraw from the study, we will still retain and analyze the data that you provided up until the point of discontinuation. At any particular point in the study, you may refuse to answer any particular question or stop participation altogether.

Questions about the Study: If you have any questions about the research now, please ask. If you have questions later about the research, you may contact: Melina Ovanessian, MA Student, 416979-5000 extension 2188 or Dr. Naomi Koerner, Research Supervisor, 416-979-5000 extension 2151.

If you have questions regarding your rights as a human participant in this study, you may contact the Ryerson University Research Ethics Board for information.

Research Ethics Board

c/o Office of the Vice President, Research and Innovation

Ryerson University

350 Victoria Street

Toronto, ON M5B 2K3

416-979-5042 


\section{Agreement:}

Your signature below indicates: (1) that you have read the information in this agreement and have had a chance to ask any questions you have about the Worry and Writing study; (2) that you agree that information collected from you during the telephone screen for the Worry and Writing study can be retained and analyzed and (3) that you agree to be in the Worry and Writing study as described in this consent form, and have been told that you can change your mind and withdraw your consent to participate at any time and that data that you have provided up to the point of your discontinuation will be retained and analyzed. You have been given a copy of this agreement.

You have been told that by signing this consent agreement you are not giving up any of your legal rights.

Name of Participant (please print)

Signature of Participant

Date

Signature of Researcher Who Obtained Informed Consent Date

Your signature below indicates that you have read and understood that you will be audiorecorded only for the purposes of this study. Your signature indicates that you agree to be audiorecorded and have been told that you can change your mind and withdraw this consent at any time.

Name of Participant (please print)

Signature of Participant

Date

Signature of Researcher Who Obtained Informed Consent Date 


\section{Agreement:}

Your signature below indicates: (1) that you have read the information in this agreement and have had a chance to ask any questions you have about the Worry and Writing study; (2) that you agree that information collected from you during the telephone screen for the Worry and Writing study can be retained and analyzed and (3) that you agree to be in the Worry and Writing study as described in this consent form, and have been told that you can change your mind and withdraw your consent to participate at any time and that data that you have provided up to the point of your discontinuation will be retained and analyzed. You have been given a copy of this agreement.

You have been told that by signing this consent agreement you are not giving up any of your legal rights.

Name of Participant (please print)

Signature of Participant

Date

Signature of Researcher Who Obtained Informed Consent Date

Your signature below indicates that you have read and understood that you will be audiorecorded only for the purposes of this study. Your signature indicates that you agree to be audiorecorded and have been told that you can change your mind and withdraw this consent at any time.

Name of Participant (please print)

Signature of Participant

Date

Signature of Researcher Who Obtained Informed Consent Date 


\section{Appendix F- Debriefing Form}

\section{Worry and Writing Study}

Everyone worries from time to time. But for people who engage in chronic worry, the worry occurs more days than not, and it usually concerns events that have not actually happened and that have a low possibility of occurring (for example, ending up homeless because of an error made at work). Thoughts of these hypothetical events can be experienced as frightening and intolerable. We conducted a study that showed that when people who worry chronically write repeatedly about threatening future events as if they are occurring in the here and now, this has a positive short-term impact on worry and anxiety. Now, we are looking at ways to strengthen the procedure and are also trying to understand how writing helps people who worry. For example, one possibility is that writing about feared situations helps a person "face" her/his fears head on.

In the study you took part in, we are wondering whether asking people to "rescript" their worst fear coming true may boost the effectiveness of our existing writing procedure. To address this question, people taking part in this study are assigned to one of three writing conditions: (1) a condition in which people write about the same "worst case scenario" three days in a row; (2) a condition in which people write about their "worst case scenario" and how they would move forward if it occurred; and (3) a condition in which people write about a neutral topic, which will help us figure out whether it is just the act of writing in and of itself that is the helpful ingredient.

Your willingness to participate in this study is greatly appreciated. This study will advance our understanding of ways that chronic worry can be managed and alleviated. Although we are interested in the potential of writing as an intervention for worry, please note that the procedure you took part in is not an actual therapy for worry at this time and shouldn't be treated as such. If you are interested in treatment for your worry, please turn over this page for a list of self-help and community resources

Contact Information: If you have any questions or concerns about this study or your participation in this study, you may contact:

Melina Ovanessian

Department of Psychology

Ryerson University

350 Victoria Street

Toronto, ON M5B 2K3

(416) $979-5000 \times 2188$

movaness@psych.ryerson.ca
Naomi Koerner

Department of Psychology

Ryerson University 350 Victoria Street

Toronto, ON M5B 2K3

(416) 979-5000 x2151

naomi.koerner@psych.ryerson.ca
Dr. Lynn Lavallee

Chair; Research Ethics Board

Ryerson University 350 Victoria Street

Toronto, Ontario, M5B 2K3

(416) 979-5000 x6300

If you would like any information about the results of the study once it is complete, please contact Melina Ovanessian or Dr. Naomi Koerner.

A note about disclosure: In order to maintain the integrity of this research, we ask that you not disclose the purpose of this study to others who may be interested in taking part. When participants have too much prior knowledge about the purpose of a study, this can affect how they behave in the study and the data for that person may not be usable. 


\section{References}

Alexander, M. J., Haugland, G., Lin, S. P., Bertollo, D. N., \& McCorry, F. A. (2008). Mental health screening in addiction, corrections and social service settings: Validating the MMS. International Journal of Mental Health and Addiction, 6, 105-119.

Alonso, J., Petukhova, M., Vilagut, G., Chatterji, S., Heeringa, S., Üstün, T. B., . . Kessler, R. C. (2011). Days out of role due to common physical and mental conditions: Results from the WHO World Mental Health surveys. Molecular Psychiatry, 16, 1234-1246.

American Psychiatric Association. (2013). Diagnostic and statistical manual for mental disorders (5th ed.). Arlington, VA: America Psychiatric Publishing.

Arntz, A., \& Weertman, A. (1999). Treatment of childhood memories: Theory and practice. Behaviour Research and Therapy, 37, 715-740.

Arntz, A., Kindt, M., \& Tiesema, M. (2007). Treatment of PTSD: A comparison of imaginal exposure with and without imagery rescripting. Journal of Behavior Therapy and Experimental Psychiatry, 38, 345-370.

Barr, D. J., Levy, R., Scheepers, C., \& Tily, H. J. (2013). Random effects structure for confirmatory hypothesis testing: Keep it maximal. Journal of Memory and Language, 68, $255-278$.

Beck, A. T. (1976). Cognitive therapy and the emotional disorders. Oxford, UK: International Universities Press.

Behar, E., Alcaine, O., Zuellig, A. R., \& Borkovec, T. D. (2003). Screening for generalized anxiety disorder using the Penn State Worry Questionnaire: A receiver operating characteristic analysis. Journal of Behavior Therapy and Experimental Psychiatry, 34, $25-43$. 
Berenbaum, H., Thompson, R. J., \& Bredemeier, K. (2007). Perceived threat: Exploring its association with worry and its hypothesized antecedents. Behaviour Research and Therapy, 45, 2473-2482.

Berg, C. Z., Shapiro, N., Chambless, D. L., \& Ahrens, A. H. (1998). Are emotions frightening? II: An analogue study of fear of emotion, interpersonal conflict, and panic onset. Behaviour Research and Therapy, 36, 3-15.

Blease, C. R., Lilienfeld, S. O., \& Kelley, J. M. (2016). Evidence-based practice and psychological treatments: The imperatives of informed consent. Frontiers in Psychology, 7, 1170.

Borkovec, T. D. (1985). Worry: A potentially valuable concept. Behaviour Research and Therapy, 23, 481-482.

Borkovec, T. D., \& Costello, E. (1993). Efficacy of applied relaxation and cognitive-behavioral therapy in the treatment of generalized anxiety disorder. Journal of Consulting and Clinical Psychology, 61, 611-619.

Borkovec, T. D., \& Roemer, L. (1995). Perceived functions of worry among generalized anxiety disorder subjects: Distraction from more emotionally distressing topics? Journal of Behavior Therapy and Experimental Psychiatry, 26, 25-30.

Borkovec, T. D., Alcaine, O., \& Behar, E. S. (2004). Avoidance theory of worry and generalized anxiety disorder. In R. Heimberg, D. Mennin \& C. Turk (Eds.), Generalized anxiety disorder: Advances in research and practice (pp. 77-108). New York, NY: Guilford Press.

Bradley, M. M., \& Lang, P. J. (1994). Measuring emotion: The Self-Assessment Manikin and the semantic differential. Journal of Behavior Therapy and Experimental Psychiatry, 25, 49- 
59.

Buhr, K., \& Dugas, M. J. (2002). The Intolerance of Uncertainty Scale: Psychometric properties of the English version. Behaviour Research and Therapy, 40, 931-946.

Buhr, K., \& Dugas, M. J. (2006). Investigating the construct validity of intolerance of uncertainty and its unique relationship with worry. Journal of Anxiety Disorders, 20, 222236.

Burton, C. M., \& King, L. A. (2009). The health benefits of writing about positive experiences: The role of broadened cognition. Psychology and Health, 24, 867-879.

Butler, G., \& Mathews, A. (1983). Cognitive processes in anxiety. Advances in Behaviour Research and Therapy, 5, 51-62.

Cheong, J., MacKinnon, D. P., \& Khoo, S. T. (2003). Investigation of mediational processes using parallel process latent growth curve modeling. Structural Equation Modeling: A Multidisciplinary Journal, 10, 238-262.

Cooper, M. (2011). Working with imagery to modify core beliefs in people with eating disorders: A clinical protocol. Cognitive and Behavioral Practice, 18, 454-465.

Craske, M. G., Barlow, D. H., \& O'Leary, T. A. (1992). Mastery of your anxiety and worry: Client Workbook. New York, NY: Oxford University Press.

Craske, M. G., \& Barlow, D. H. (2006). Mastery of your anxiety and panic: Therapist guide (3rd ed.). New York, NY: Oxford University Press.

Cuijpers, P. (2016). Are all psychotherapist equally effective in the treatment of adult depression? The lack of statistical power of comparative outcome studies. EvidenceBased Mental Health, 19, 39-42.

Davey, G. C., Hampton, J., Farrell, J., \& Davidson, S. (1992). Some characteristics of worrying: 
Evidence for worrying and anxiety as separate constructs. Personality and Individual Differences, 13, 133-147.

Davey, G. C. L. (1994). Worrying, social problem-solving abilities, and social problem-solving confidence. Behaviour Research and Therapy, 32, 327-330.

Davey, G. C. L., \& Levy, S. (1998). Catastrophic worrying: Personal inadequacy and a perseverative iterative style as features of the catastrophizing process. Journal of Abnormal Psychology, 107, 576-586.

Deschenes, S., Dugas, M. J., Fracalanza, K., \& Koerner, N. (2012). The role of anger in generalized anxiety disorder. Cognitive Behaviour Therapy, 41, $261-271$.

Decker, M. L., Turk, C. L., Hess, B., \& Murray, C. E. (2008). Emotion regulation among individuals classified with and without generalized anxiety disorder. Journal of Anxiety Disorders, 22, 485-494.

Dimidjian, S., Barrera, J., Manuel, Martell, C., Muñoz, R. F., \& Lewinsohn, P. M. (2011). The origins and current status of behavioral activation treatments for depression. Annиаl Review of Clinical Psychology, 7, 1-38.

Di Nardo, P. A., Brown, T. A. \& Barlow D. H. (1994). Anxiety Disorder Interview Schedule for DSM-IV: Lifetime version (ADIS-IV-L). New York, NY: Oxford University Press.

Donegan, E., \& Dugas, M. J. (2012). Generalized anxiety disorder: A comparison of symptom change in adults receiving cognitive-behavioral therapy or applied relaxation. Journal of Consulting and Clinical Psychology, 80, 490-496.

Dugas, M. J., Brillon, P., Savard, P., Turcotte, J., Gaudet, A., Ladouceur, R., . . Gervais, N. J. (2010). A randomized clinical trial of cognitive-behavioral therapy and applied relaxation for adults with generalized anxiety disorder. Behavior Therapy, 41, 46-58. 
Dugas, M. J., \& Koerner, N. (2005). Cognitive-behavioral treatment for generalized anxiety disorder: Current status and future directions. Journal of Cognitive Psychotherapy, 19, $61-81$.

Dugas, M. J., \& Robichaud, M. (2007). Cognitive-behavioral treatment for generalized anxiety disorder: From science to practice. New York, NY: Routledge.

Dugas, M. J., Buhr, K., \& Ladouceur, R. (2004). The role of intolerance of uncertainty in etiology and maintenance. In R. G. Heimberg, C. L. Turk, \& D. S. Mennin (Eds.), Generalized anxiety disorder: Advances in research and practice (pp. 143-163). New York, NY: Guilford Press.

Dugas, M. J., Freeston, M. H., \& Ladouceur, R. (1997). Intolerance of uncertainty and problem orientation in worry. Cognitive Therapy and Research, 21, 593-606.

Dugas, M. J., Freeston, M. H., Ladouceur, R., Rhéaume, J., Provencher, M., \& Boisvert, J. (1998). Worry themes in primary GAD, secondary GAD, and other anxiety disorders. Journal of Anxiety Disorders, 12, 253-261.

Dugas, M. J., Gagnon, F., Ladouceur, R., \& Freeston, M. H. (1998). Generalized anxiety disorder: A preliminary test of a conceptual model. Behaviour Research and Therapy, 36, $215-226$.

Dugas, M. J., Letarte, H., Rhéaume, J., Freeston, M. H., \& Ladouceur, R. (1995). Worry and problem solving: Evidence of a specific relationship. Cognitive Therapy and Research, 19, 109-120.

Dugas, M. J., Savard, P., Gaudet, A., Turcotte, J., Laugesen, N., Robichaud, M., . . Koerner, N. (2007). Can the components of a cognitive model predict the severity of generalized anxiety disorder? Behavior Therapy, 38, 169-178. 
D'Zurilla, T. J., \& Goldfried, M. R. (1971). Problem solving and behavior modification. Journal of Abnormal Psychology, 78, 107-126.

Eagleson, C., Hayes, S., Mathews, A., Perman, G., \& Hirsch, C. R. (2016). The power of positive thinking: Pathological worry is reduced by thought replacement in generalized anxiety disorder. Behaviour Research and Therapy, 78, 13-18.

Eaton, W. W., Muntaner, C., Smith, C., Tien, A., \& Ybarra, M. (2004). Center for Epidemiologic Studies Depression Scale: Review and revision (CESD and CESD-R). In M. E. Maruish (Ed.), The use of psychological testing for treatment planning and outcomes assessment (pp. 363-377). Mahwah, NJ: Lawrence Erlbaum Associates.

Fisher, A. J., \& Nordberg, S. S. (2008). An open trial of integrative therapy for generalized anxiety disorder. Psychotherapy: Theory, Research, Practice, Training, 45, 135-147.

Foa, E. B., \& Kozak, M. J. (1986). Emotional processing of fear: Exposure to corrective information. Psychological Bulletin, 99, 20-35.

Foa, E. B., Riggs, D. S., Massie, E. D., \& Yarczower, M. (1995). The impact of fear activation and anger on the efficacy of exposure treatment for posttraumatic stress disorder. Behavior Therapy, 26, 487-499.

Foa, E. B., \& Rothbaum, B. A. (1998). Treating the trauma of rape: Cognitive behavioral therapy for PTSD. New York, NY: Guilford Press.

Fracalanza, K. (2010). The impact of exposure on generalized anxiety disorder-related symptoms and cognitive processes: The role of stimulus variation. (Master's thesis, Ryerson University, Toronto, Canada). 
Fracalanza, K., Koerner, N., \& Antony, M. M. (2014). Testing a procedural variant of written imaginal exposure for generalized anxiety disorder. Journal of Anxiety Disorders, 28, 559-569.

Frattaroli, J. (2006). Experimental disclosure and its moderators: A meta-analysis. Psychological Bulletin, 132, 823-865.

Freeston, M. H., Dugas, M. J., \& Ladouceur, R. (1996). Thoughts, images, worry, and anxiety. Cognitive Therapy and Research, 20, 265-273.

Freeston, M. H., Rhéaume, J., Letarte, H., Dugas, M. J., \& Ladouceur, R. (1994). Why do people worry? Personality and Individual Differences, 17, 791-802.

Furr, R. M. (2008). A contrast analysis approach to change. Educational Research and Evaluation, 14, 335-362.

Gallop, R., \& Tasca, G. A. (2009). Multilevel modeling of longitudinal data for psychotherapy researchers: II. the complexities. Psychotherapy Research, 19, 438-452.

Goldman, N., Dugas, M. J., Sexton, K. A., \& Gervais, N. J. (2007). The impact of written exposure on worry: A preliminary investigation. Behavior Modification, 31, 512-538.

Gosselin, P., Pelletier, O., \& Ladouceur, R. (2001, July). The Negative Problem Orientation Questionnaire (NPOQ): Development and validation among a non-clinical sample. Poster presented at the meeting of the World Congress of Behavioural and Cognitive Therapies, Vancouver, BC.

Gould, R. A., Safren, S. A., Washington, D. O., \& Otto, M. W. (2004). A meta-analytic review of cognitive-behavioral treatments. In R. G. Heimberg, C. L. Turk, \& D. S. Mennin (Eds.), Generalized anxiety disorder: Advances in research and practice (pp. 248-264). New York, NY: Guilford Press. 
Grös, D. F., Antony, M. M., Simms, L. J., \& McCabe, R. E. (2007). Psychometric properties of the State-Trait Inventory for Cognitive and Somatic Anxiety (STICSA): Comparison to the State-Trait Anxiety Inventory (STAI). Psychological Assessment, 19, 369-381.

Gross, J. J., \& Thompson, R. (2007). Emotion regulation: Conceptual foundations. In J. J. Gross (Ed.), Handbook of emotion regulation (pp. 3 - 24). New York, NY: Guilford Press.

Grunert, B. K., Weis, J. M., Smucker, M. R., \& Christianson, H. (2007). Imagery rescripting and reprocessing therapy after failed prolonged imaginal exposure for posttraumatic stress disorder following industrial injury. Journal of Behavior Therapy and Experimental Psychiatry, 7, 173-182.

Grunnert, B. K., Smucker, M. R., Weis, J. M., \& Rusch, M. D. (2003). When prolonged exposure fails: Adding an imagery-based cognitive restructuring component in the treatment of industrial accident victims suffering from PTSD. Cognitive and Behavioral Practice, 10, 333-346.

Hagenaars, M. A., \& Arntz, A. (2012). Reduced Intrusion Development after Post-Trauma Imagery Rescripting: An experimental study. Journal of Behavior Therapy and Experimental Psychiatry, 43, 808-814.

Hayes, S. C., Wilson, K. G., Gifford, E. V., Follette, V. M., \& Strosahl, K. (1996). Experiential avoidance and behavioral disorders: A functional dimensional approach to diagnosis and treatment. Journal of Consulting and Clinical Psychology, 64, 1152-1168.

Hancock, G. R., \& Klockars, A. J. (1996). The quest for $\alpha$ : Developments in multiple comparison procedures in the quarter century since games (1971). Review of Educational Research, 66, 269-306.

Hanrahan, F., Field, A. P., Jones, F. W., \& Davey, G. C. L. (2013). A meta-analysis of cognitive 
therapy for worry in generalized anxiety disorder. Clinical Psychology Review, 33, 120132.

Henning, E. R., Turk, C. L., Mennin, D. S., Fresco, D. M., \& Heimberg, R. G. (2007). Impairment and quality of life in individuals with generalized anxiety disorder. Depression and Anxiety, 24, 342-349.

Hesser, H. (2015). Modeling individual differences in randomized experiments using growth models: Recommendations for design, statistical analysis and reporting of results of internet interventions. Internet Interventions, 2, 110-120.

Hirsch, C. R., \& Mathews, A. (2012). A cognitive model of pathological worry. Behaviour Research and Therapy, 50, 636-646.

Hirsch, C. R., Mathews, A., Lequertier, B., Perman, G., \& Hayes, S. (2013). Characteristics of worry in generalized anxiety disorder. Journal of Behavior Therapy and Experimental Psychiatry, 44, 388-395.

Holmes, E. A., \& Mathews, A. (2005). Mental imagery and emotion: A special relationship? Emotion, 5, 489-497.

Holmes, E. A., Arntz, A., \& Smucker, M. R. (2007). Imagery rescripting in cognitive behaviour therapy: Images, treatment techniques and outcomes. Journal of Behavior Therapy and Experimental Psychiatry, 38, 297-305.

Holmes, E. A., \& Mathews, A. (2010). Mental imagery in emotion and emotional disorders. Clinical Psychology Review, 30, 349-362.

Hoyer, J., Beesdo, K., Gloster, A. T., Runge, J., Höfler, M., \& Becker, E. S. (2009). Worry exposure versus applied relaxation in the treatment of generalized anxiety disorder. Psychotherapy and Psychosomatics, 78, 106-115. 
Hunt, M. G., \& Fenton, M. (2007). Imagery rescripting versus in vivo exposure in the treatment of snake fear. Journal of Behavior Therapy and Experimental Psychiatry, 38, 329-344.

Keller, M. B., McCullough, J. P., Klein, D. N., Arnow, B., Dunner, D. L., Gelenberg, A. J., . . . Zajecka, J. (2000). A comparison of nefazodone, the cognitive behavioral-analysis system of psychotherapy, and their combination for the treatment of chronic depression. New England Journal of Medicine, 342, 1462-1470.

Kessler, R. C., Berglund, P., Demler, O, Jin, R., Merikandas, K. R., \& Walters, E. E. (2005). Lifetime prevalence and age-of-onset distributions of DSM-IV disorders in the National Comorbidity Survey Replication. Archives of General Psychiatry 62, 593-602.

Kessler, R. C., Chiu, W. T., Demler, O., Merikangas, K. R., \& Walters, E. E. (2005). Prevalence, severity, and comorbidity of 12-month DSM-IV disorders in the National Comorbidity Survey Replication. Archives of General Psychiatry 62, 617-627.

King, L. A. (2001). The health benefits of writing about life goals. Personality and Social Psychology Bulletin, 27, 798-807.

Koerner, N., \& Dugas, M. J. (2006). A cognitive model of generalized anxiety disorder: the role of intolerance of uncertainty. In G. Davey \& A. Wells (Eds.), Worry and its psychological disorders: Theory, assessment and treatment (pp. 201-216). West Sussex, UK: John Wiley and Sons.

Koerner, N., \& Fracalanza, K. (2012). The role of anxiety control strategies in imaginal exposure. In P. Neudeck \& H-U Wittchen (Eds.), Exposure therapy: Rethinking the model-Refining the method (pp. 197-216). New York, NY: Springer Press. 
Kraemer, H. C., Wilson, G. T., Fairburn, C. G., \& Agras, W. S. (2002). Mediators and moderators of treatment effects in randomized clinical trials. Archives of General Psychiatry, 59, 877-883.

Ladouceur, R., Blais, F., Freeston, M. H., \& Dugas, M. J. (1998). Problem solving and problem orientation in generalized anxiety disorder. Journal of Anxiety Disorders, 12, 139-152.

Lecrubier, Y., Sheehan, D. V., Weiller, E., Amorim, P., Bonora, I., Sheehan, K. H.,...Dunbar, G. C. (1997). The Mini International Neuropsychiatric Interview (MINI): A short diagnostic structured interview: Reliability and validity according to the CIDI. European Psychiatry, 12, 224-231.

Lang, P. J., Levin, D. N., Miller, G. A., \& Kozak, M. J. (1983). Fear behavior, fear imagery, and the psychophysiology of emotion: The problem of affective response integration. Journal of Abnormal Psychology, 92, 276-306.

Long, M. E., Hammons, M. E., Davis, J. L., Frueh, B. C., Khan, M. M., Elhai, J. D., \& Teng, E. J. (2011). Imagery rescripting and exposure group treatment of posttraumatic nightmares in veterans with PTSD. Journal of Anxiety Disorders, 25, 531-535.

Long, M. E., \& Quevillon, R. P. (2009). Imagery rescripting in the treatment of posttraumatic stress disorder. Journal of Cognitive Psychotherapy. 23, 67-76.

Lumley, M. A., \& Provenzano, K. M. (2003). Stress management through written emotional disclosure improves academic performance among college students with physical symptoms. Journal of Educational Psychology, 95, 641-649.

MacKinnon, D. P. (2008). Introduction to statistical mediation analysis Lawrence Erlbaum Associates. 
MacLeod, A. K., Williams, J. M., \& Bekerian, D. A. (1991). Worry is reasonable: The role of explanation in pessimism about future personal events. Journal of Abnormal Psychology, $100,478-486$.

Maric, M., Wiers, R. W., \& Prins, P. J. M. (2012). Ten ways to improve the use of statistical mediation analysis in the practice of child and adolescent treatment research. Clinical Child and Family Psychology Review, 15, 177-191.

Martens, E. J., De Jonge, P.,Na, B., Cohen, B. E., Lett, H., \& Whooley, M. A. (2010). Scared to death? Generalized anxiety disorder and cardiovascular events in patients with stable coronary heart disease: The Heart and Soul Study. Archives of General Psychiatry 67, $750-758$.

McEvoy, P. M., Erceg-Hurn, D. M., Saulsman, L. M., \& Thibodeau, M. A. (2015). Imagery enhancements increase the effectiveness of cognitive behavioural group therapy for social anxiety disorder: A benchmarking study. Behaviour Research and Therapy, 65, 42-51.

Mennin, D. S. (2004). Emotion regulation therapy for generalized anxiety disorder. Clinical Psychology \& Psychotherapy, 11, 17-29.

Mennin, D. S., Heimberg, R. G., Turk, C. L., \& Fresco, D. M. (2005). Preliminary evidence for an emotion dysregulation model of generalized anxiety disorder. Behaviour Research and Therapy, 43, 1281-1310.

Mennin, D. S., Holaway, R. M., Fresco, D. M., Moore, M. T., \& Heimberg, R. G. (2007). Delineating components of emotion and its dysregulation in anxiety and mood psychopathology. Behavior Therapy, 38, 284-302. 
Mennin, D. S., McLaughlin, K. A., \& Flanagan, T. J. (2009). Emotion regulation deficits in generalized anxiety disorder, social anxiety disorder, and their co-occurrence. Journal of Anxiety Disorders, 23, 866-871.

Mennin, D. S., Turk, C. L., Heimberg, R. G., \& Carmin, C. (2004). Focusing on the regulation of emotion: A new direction for conceptualizing generalized anxiety disorder. In M. A. Reinecke \& D. A. Clark (Eds.), Cognitive therapy over the lifespan: Evidence and practice (pp. 60-89). New York, NY: Cambridge University Press.

Metzger, R. L., Miller, M. L., Cohen, M., \& Sofka, M. (1990). Worry changes decision making: The effect of negative thoughts on cognitive processing. Journal of Clinical Psychology, $46,78-88$.

Meyer, T. J., Miller, M. L., Metzger, R. L., \& Borkovec, T. D. (1990). Development and validation of the Penn State Worry Questionnaire. Behaviour Research and Therapy, 28, 487-495.

Molina, S., \& Borkovec, T. D. (1994). The Penn State Worry Questionnaire: Psychometric properties and associated characteristics. In G. C. L. Davey, \& F. Tallis (Eds.), Worrying: Perspectives on theory, assessment and treatment. (pp. 265-283). Oxford, UK: John Wiley and Sons.

Moore, M. T., Anderson, N. L., Barnes, J. M., Haigh, E. A. P., \& Fresco, D. M. (2014). Using the GAD-Q-IV to identify generalized anxiety disorder in psychiatric treatment seeking and primary care medical samples. Journal of Anxiety Disorders, 28, 25-30.

Morina, N., Lancee, J., \& Arntz, A. (2017). Imagery rescripting as a clinical intervention for aversive memories: A meta-analysis. Journal of Behavior Therapy and Experimental Psychiatry, 55, 6-15. 
Moscovitch, D. A., Chiupka, C. A., \& Gavric, D. L. (2013). Within the mind's eye: Negative mental imagery activates different emotion regulation strategies in high versus low socially anxious individuals. Journal of Behavior Therapy and Experimental Psychiatry, $44,426-432$.

Mota, N. P., Schaumberg, K., Vinci, C., Sippel, L. M., Jackson, M., Schumacher, J. A., \& Coffey, S. F. (2015). Imagery vividness ratings during exposure treatment for posttraumatic stress disorder as a predictor of treatment outcome. Behaviour Research and Therapy, 69, 22-28.

Newman, M. G., Castonguay, L. G., Borkovec, T. D., \& Molnar, C. (2004). Integrative psychotherapy. In R. G. Heimberg, C. L. Turk, D. S. Mennin (Eds.), Generalized Anxiety Disorder: Advances in research and practice (pp. 320-350). New York, NY: Guilford Press.

Newman, M. G., \& Llera, S. J. (2011). A novel theory of experiential avoidance in generalized anxiety disorder: A review and synthesis of research supporting a contrast avoidance model of worry. Clinical Psychology Review, 31, 371-382.

Newman, M. G., Zuellig, A. R., Kachin, K. E., Constantino, M. J., Przeworski, A., Erickson, T., \& Cashman-McGrath, L. (2002). Preliminary reliability and validity of the Generalized Anxiety Disorder Questionnaire-IV: A revised self-report diagnostic measure of generalized anxiety disorder. Behavior Therapy, 33, 215-233.

Paivio, A., \& Marschark, M. (1991). Integrative processing of concrete and abstract sentences. In A. Paivio (Ed.), Images in the mind: The evolution of a theory (pp. 134-154). New York, NY: Harvester Wheatsheaf. 
Pawluk, E. J., Koerner, N., \& Antony, M. M. (2011, November). Impact of concreteness training on worry, intolerance of uncertainty, and problem solving. Poster presented at the meeting of the Association for Behavioral and Cognitive Therapies Toronto, ON.

Pennebaker, J. W., Booth, R. J., \& Francis, M. E. (2007). Linguistic Inquiry and Word Count (LIWC): LIWC2007. Austin, TX: LIWC.net.

Preacher, K. J., \& Hayes, A. F. (2004). SPSS and SAS procedures for estimating indirect effects in simple mediation models. Behavior Research Methods, Instruments, and Computers, $36,717-731$.

Provencher, M. D., Dugas, M. J., \& Ladouceur, R. (2004). Efficacy of problem-solving training and cognitive exposure in the treatment of generalized anxiety disorder: A case replication series. Cognitive and Behavioral Practice, 11, 404-414.

Provencher, M. D., Freeston, M. H., Dugas, M. J., \& Ladouceur, R. (2000). Catastrophizing assessment of worry and threat schemata among worriers. Behavioural and Cognitive Psychotherapy, 28, 211-224.

Radcliffe, A. M., Lumley, M. A., Kendall, J., Stevenson, J. K., \& Beltran, J. (2007). Written emotional disclosure: Testing whether social disclosure matters. Journal of Social and Clinical Psychology, 26, 362-384.

Radloff, L. S. (1977). The CES-D scale: A self-report depression scale for research in the general population. Applied Psychological Measurement, 1, 385-401.

Raudenbush, S. W., Bryk, A. S., Cheong, Y. F., Congdon, R., \& du Toit, M. (2004). HLM6: Hierarchical linear and non-linear modeling. Lincolnwood, IL: Scientific Software International.

Ree, M. J., French, D., MacLeod, C., \& Locke, V. (2008). Distinguishing cognitive and somatic 
dimensions of state and trait anxiety: Development and validation of the State-Trait Inventory for Cognitive and Somatic Anxiety (STICSA). Behavioural and Cognitive Psychotherapy, 36, 313-332.

Robichaud, M., \& Dugas, M. J. (2005). Negative problem orientation (Part I): Psychometric properties of a new measure. Behaviour Research and Therapy, 43, 391-401.

Robichaud, M., \& Dugas, M. J. (2006). A cognitive-behavioral treatment targeting intolerance of uncertainty. In G. Davey \& A. Wells (Eds.), Worry and its psychological disorders: theory, assessment and treatment (pp. 289-304). West Sussex, UK: John Wiley and Sons.

Robichaud, M., \& Dugas, M. J. (2015). The Generalized anxiety disorder workbook: A comprehensive CBT guide for coping with uncertainty, worry, and fear. Oakland, CA: New Harbinger Publications.

Robinson, C. M., Klenck, S. C., \& Norton, P. J. (2010). Psychometric properties of the Generalized Anxiety Disorder Questionnaire for DSM-IV among four racial groups. Cognitive Behaviour Therapy, 39, 251-261.

Roemer, L., Salters, K., Raffa, S. D., \& Orsillo, S. M. (2005). Fear and avoidance of internal experiences in GAD: Preliminary tests of a conceptual model. Cognitive Therapy and Research, 29, 71-88.

Rogosa, D. R. (1988). Myths about longitudinal research. In K. W. Schaie, R. T. Campbell, W. Meredith, \& S. C. Rawlings (Eds.), Methodological issues in aging research (pp. 171210). New York, NY: Springer Press.

Rusch, M. D., Grunert, B. K., Mendelsohn, R. A., \& Smucker, M. R. (2000). Imagery rescripting for recurrent, distressing images. Cognitive and Behavioral Practice, 7, 173-182. 
Schacter, D. L. (2012). Adaptive constructive processes and the future of memory. American Psychologist, 67, 603-613.

Schafer, J. L. (1999). Multiple imputation: A primer. Statistical Methods in Medical Research, 8, 3-15.

Sexton, K. A., \& Dugas, M. J. (2008). The Cognitive Avoidance Questionnaire: Validation of the English translation. Journal of Anxiety Disorders, 22, 355-370.

Sexton, K. A., \& Dugas, M. J. (2009). Defining distinct negative beliefs about uncertainty: Validating the factor structure of the Intolerance of Uncertainty Scale. Psychological Assessment, 21, 176-186.

Shah, J. Y., \& Kruglanski, A. W. (2008). Structural Dynamics: The challenge of change in goal systems. In J. Y. Shah, \& W. L. Gardner (Eds.), Handbook of motivational science (pp. 217-229). New York, NY: Guilford Press.

Shahmoradi, G. (2013). The effectiveness of worry exposure in treating generalized anxiety disorder. Management Science Letters, 3, 1659-1664.

Sharpe, J. P., \& Gilbert, D. G. (1998). Effects of repeated administration of the Beck Depression Inventory and other measures of negative mood states. Personality and Individual Differences, 24, 457-463.

Sheehan, D. V., Lecrubier, Y., Sheehan, K. H., Amorim, P., Janavs, J., Weiller, E.,...Dunbar, G. C. (1998). The Mini-International Neuropsychiatric Interview (M.I.N.I): The development and validation of a structured diagnostic psychiatric interview for DSM-IV and ICD-10. Journal of Clinical Psychiatry, 59, 22-33.

Sheehan, D. V. (2015). Mini International Neuropsychiatric Interview 7.0. Jacksonville, FL: Medical Outcomes Systems.

Sloan, D. M., Marx, B. P., \& Epstein, E. M. (2005). Further examination of the exposure model 
underlying the efficacy of written emotional disclosure. Journal of Consulting and Clinical Psychology, 73, 549-554.

Smucker, M. R., Dancu, C. V., Foa, E. B., \& Niederee, J. L. (1995). Imagery rescripting: A new treatment for survivors of childhood sexual abuse suffering from post-traumatic stress. Journal of Cognitive Psychotherapy: An International Quarterly, 9, 3-17.

Stapinski, L. A., Abbott, M. J., \& Rapee, R. M. (2010). Fear and perceived uncontrollability of emotion: Evaluating the unique contribution of emotion appraisal variables to prediction of worry and generalised anxiety disorder. Behaviour Research and Therapy, 48, 10971104.

Stöber, J. (1998).Worry, problem elaboration and suppression of imagery: The role of concreteness. Behaviour Research and Therapy, 36, 751-756.

Stöber, J. (2000). Worry, thoughts, and images: A new conceptualization. In U. von Hecker, S. Dutke \& G. Sedek (Eds.), Generative mental processes and cognitive resources: Integrative research on adaptation and control (pp. 223-244). Dordrecht, Netherlands: Kluwer.

Stöber, J., \& Borkovec, T. D. (2002). Reduced concreteness of worry in generalized anxiety disorder: Findings from a therapy study. Cognitive Therapy and Research, 26, 89-96.

Stöber, J., \& Bittencourt, J. (1998). Weekly assessment of worry: An adaptation of the Penn State Worry Questionnaire for monitoring changes during treatment. Behaviour Research and Therapy, 36, 645-656.

Stöber, J., Tepperwien, S., \& Staak, M. (2000). Worrying leads to reduced concreteness of problem elaborations: Evidence for the avoidance theory of worry. Anxiety, Stress and Coping, 13, 217-227. 
Tabachnick, B. G., and Fidell, L. S. (2013). Using multivariate statistics, 6th ed. Boston, MA: Pearson.

Turk, C. L., Heimberg, R. G., Luterek, J. A., Mennin, D. S., \& Fresco, D. M. (2005). Emotion dysregulation in generalized anxiety disorder: A comparison with social anxiety disorder. Cognitive Therapy and Research, 29, 89-106.

Tyrer, P., \& Baldwin, D. (2006). Generalised anxiety disorder. The Lancet, 368, 2156-2166.

Van Dam, N. T., \& Earleywine, M. (2011). Validation of the center for epidemiologic studies depression Scale-Revised (CESD-R): Pragmatic depression assessment in the general population. Psychiatry Research, 186, 128-132.

van der Heiden, C., \& ten Broeke, E. (2009). The when, why, and how of worry exposure. Cognitive and Behavioral Practice, 16, 386-393.

van Tilburg, W., Dirksen, C., van Asselt, T., Giesen-Bloo, J., Spinhoven, P., van Dyck, R., ... Nadort, M. (2006) Outpatient psychotherapy for borderline personality disorder: A randomized clinical trial of schema focused therapy versus transference focused psychotherapy. Archives of General Psychiatry, 63, 649-658.

Vasey, M. W., \& Borkovec, T. D. (1992). A catastrophizing assessment of worrisome thoughts. Cognitive Therapy and Research, 16, 505-520.

Waters, A. M. \& Craske, M. G. (2005). Generalized anxiety disorder. In: M. M. Antony, D. R. Ledley, \& R. G. Heimberg (Eds.), Improving outcomes and preventing relapse in cognitive behavioral therapy (pp.77-128). New York, NY: Guilford Press.

Watkins, E. (2004). Adaptive and maladaptive ruminative self-focus during emotional processing. Behaviour Research and Therapy, 42, 1037-1052. 
Watkins, E. R., Baeyens, C. B., \& Read, R. (2009). Concreteness training reduces dysphoria: Proof-of-principle for repeated cognitive bias modification in depression. Journal of Abnormal Psychology, 118, 55-64.

Watkins, E., Moberly, N. J., \& Moulds, M. L. (2008). Processing mode causally influences emotional reactivity: Distinct effects of abstract versus concrete construal on emotional response. Emotion, 8, 364-378.

Weertman, A., \& Arntz, A. (2007). Effectiveness of treatment of childhood memories in cognitive therapy for personality disorders: A controlled study contrasting methods focusing on the present and methods of focusing on childhood memories. Behaviour Research and Therapy, 45, 2133-2143.

Wheatley, J., \& Hackmann, A. (2011). Using imagery rescripting to treat major depression: Theory and practice. Cognitive and Behavioral Practice, 18, 444-453.

Wild, J., Hackmann, A., \& Clark, D. M. (2007). When the present visits the past: Updating traumatic memories in social phobia. Journal of Behavior Therapy and Experimental Psychology, 38, 386-401.

Wild, J., Hackmann, A., \& Clark, D.M. (2008). Rescripting early memories linked to negative images in social phobia: A pilot study. Behavior Therapy, 39, 47-56.

Williams, K. E., Chambless, D. L., \& Ahrens, A.H. (1997). Are emotions frightening? An extension of the fear of fear concept. Behaviour Research and Therapy, 35, 239-248.

Wu, J. Q., Szpunar, K. K., Godovich, S. A., Schacter, D. L., \& Hofmann, S. G. (2015). Episodic future thinking in generalized anxiety disorder. Journal of Anxiety Disorders, 36, 1-8.

Young, J. E., Klosko, J. S., \& Weishaar, M. E. (2003). Schema therapy: A practitioner's guide. New York, NY: Guilford Press. 
Young, J. F., Kranzler, A., Gallop, R., \& Mufson, L. (2012). Interpersonal psychotherapyadolescent skills training: Effects on school and social functioning. School Mental Health, 4, 254-264. 